\title{
EXPERIMENTELLE UNTERSUCHUNGEN VON FINGERSTRÖMUNG UND THERMOHALINEN TREPPEN FÜR INSTABILE AUFTRIEBSVERHÄLTNISSE
}

\author{
DisSERTATION \\ zur Erlangung des mathematisch-naturwissenschaftlichen Doktorgrades \\ "Doctor rerum naturalium" \\ der Georg-August-Universität zu Göttingen \\ im Promotionsprogramm ProPhys \\ der Georg-August-University School of Science (GAUSS)
}

vorgelegt von

Matthias Kellner

aus Schönebeck/Elbe 


\section{Betreuungsausschuss}

Prof. Dr. Andreas Tilgner, Geophysikalische Strömungsmechanik, Institut für Geophysik Göttingen

Prof. Dr. Dr. Andreas Dillmann, Institut für Aerodynamik und Strömungstechnik, DLR

Göttingen

\section{Mitglieder der Prüfungskommission}

\section{Referent:}

Prof. Dr. Andreas Tilgner, Geophysikalische Strömungsmechanik, Institut für Geophysik Göttingen

Korreferent:

Prof. Dr. Dr. Andreas Dillmann, Institut für Aerodynamik und Strömungstechnik, DLR Göttingen

weitere Mitglieder der Prüfungskommission

Dr. Martin Rein, Institut für Aerodynamik und Strömungstechnik (Hochgeschwindigkeitskonfigurationen), DLR Göttingen

Prof. Dr. Sarah Köster, Forschungsgruppe "Nanoscale Imaging of Cellular Dynamics", Institut für Röntgenphysik Göttingen

Prof. Dr. Wolfgang Glatzel, Institut für Astrophysik Göttingen

Prof. Dr. Stefan Dreizler, Institut für Astrophysik Göttingen

Tag der mündlichen Prüfung: 13.04.2016 


\section{Inhaltsverzeichnis}

Abbildungsverzeichnis ii

Tabellenverzeichnis iv

1 Einleitung 1

2 Theoretische Grundüberlegungen $\quad \mathbf{5}$

2.1 Doppelt diffusive Strömung . . . . . . . . . . . . . . . . . . 5

2.1.1 Grundgleichungen und Kennzahlen . . . . . . . . . . . . . 5

2.1.2 Randbedingungen . . . . . . . . . . . . . . . . 7

2.1.3 Regime der doppelt diffusiven Konvektion . . . . . . . . . . . . 8

2.2 Thermohaline Treppen . . . . . . . . . . . . . . . . . . . . . . . . . . . . . 10

2.3 Funktionsweise der elektrochemischen Zelle . . . . . . . . . . . . . . . . 12

2.4 Messmethoden . . . . . . . . . . . . . . . . . . . . . . . 15

2.5 Fehlereinschätzung . . . . . . . . . . . . . . . . . . . . . . . 18

3 Grenzen des Fingerregimes $\quad 19$

3.1 Vorüberlegungen . . . . . . . . . . . . . . . . . . . . . . . . . 19

3.2 Das Experiment . . . . . . . . . . . . . . . . . . . . . . . . 21

3.3 Ergebnisse . . . . . . . . . . . . . . . . . . . . . 23

3.3.1 Bestimmung des Überganges . . . . . . . . . . . . . . . . . . . 23

3.3.2 Eigenschaften des Systems am Übergang . . . . . . . . . . . . . . . 28

3.3.3 Mechanismen für den Übergang . . . . . . . . . . . . . . . . . . . . 34

4 Thermohaline Treppen 41

4.1 Vorüberlegungen . . . . . . . . . . . . . . . . . . . . . 41

4.2 Das Experiment . . . . . . . . . . . . . . . . . . . . . . 44

4.3 Ergebnisse . . . . . . . . . . . . . . . . . . . . . 47

5 Zusammenfassung $\quad 59$ 
6 Nomenklatur 65

$\begin{array}{lll}7 & \text { Apendix } & 67\end{array}$

7.1 Abbildungen und Messdaten . . . . . . . . . . . . . . . . . . 67

7.2 Ergebnistabellen . . . . . . . . . . . . . . . . . . . 70

7.3 Gleichungen zur Bestimmung der Stoffeigenschaften . . . . . . . . . . . . 78

7.4 Technische Daten . . . . . . . . . . . . . . . . . . . . . . . 79

$\begin{array}{ll}\text { Literaturverzeichnis } & 85\end{array}$ 


\section{Abbildungsverzeichnis}

1.1 T-S-Profil einer thermohalinen Treppe . . . . . . . . . . . . . . . 2

2.1 Regime der doppelt diffusiven Konvektion . . . . . . . . . . . . . . 8

2.2 Turnerwinkel . . . . . . . . . . . . . . . . . . . . . . . . . . 9

2.3 Kennlinie der doppelt diffusiven Messzelle . . . . . . . . . . . . . . . . 13

2.4 Schematischer Aufbau und Funktionsweise des PIV . . . . . . . . . . . . 15

2.5 Beispiel eines Shadowgraphbildes . . . . . . . . . . . . . . . . 16

3.1 Aufbau des Versuches . . . . . . . . . . . . . . . . . . . . . 21

$3.2 R e_{x}$ und $R e_{y}$ gegen $R a_{c}$ am Übergang aufgetragen für $R a_{c}=2,92 \cdot 10^{10} \quad 24$

3.3 Strömungsverlauf am Übergang für $R a_{c}=2,92 \cdot 10^{10} \ldots \ldots$. . . . . . . 25

3.4 Geschwindigkeitsverteilung im konvektiven und im Fingerregime . . . . . 26

3.5 Übergang in der $\left|R a_{T}\right|-R a_{c^{-}}$Ebene . . . . . . . . . . . . . . . 27

3.6 Sherwoodzahl aufgetragen gegen $R a_{T}$ für $R a_{c}=2,43 \cdot 10^{9} \ldots \ldots \ldots$

3.7 Sherwoodzahl aufgetragen gegen $R a_{c}^{4 / 9} / R a_{T}^{1 / 12}$ und $R a_{c}^{0,372} / R a_{T}^{0,074} \ldots 29$

3.8 Sherwoodzahl aufgetragen gegen $R a_{c}$ bei $R a_{T}=0 \ldots \ldots$. . . . . . . 31

3.9 Reynoldszahl aufgetragen gegen $R a_{T}$ für $R a_{c}=2,43 \cdot 10^{9} \ldots \ldots$. . . . . 31

3.10 Reynoldszahl aufgetragen gegen $R a_{c} / \sqrt{R a_{T}}$ und $R a_{c}^{0.579} R a_{T}^{-0.288} \ldots . . .32$

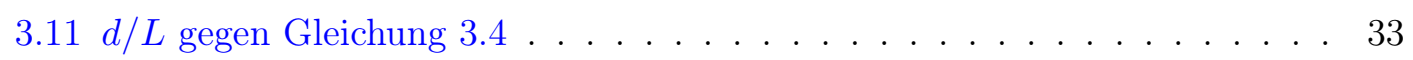

3.12 Temperaturisolinien für $R a_{c}=2,92 \cdot 10^{10}$ und $\left|R a_{T}\right|=1,86 \cdot 10^{7} \ldots 37$

3.13 Maximale und minimale Höhe der Isotherme für $R a_{c}=2,92 \cdot 10^{10} \ldots$. . . 38

3.14 Vergleich der Übergänge in der $\left|R a_{T}\right|-R a_{c^{-}}$Ebene . . . . . . . . . . . . . 39

4.1 Experimenteller Aufbau des Versuches zu thermohalinen Treppen . . . . 44

4.2 Zwei-Eimer-Methode . . . . . . . . . . . . . . . . . . . 46

4.3 Shadowgraphaufnahme einer thermohalinen Treppe . . . . . . . . . . . . 47

4.4 Geschwindigkeitsprofil für die thermohalinen Treppen . . . . . . . . . . . . 49

4.5 Geschwindigkeitsprofil im Fingerregime für Zellen mit $\Gamma=10,20 / 3$ und 10/3 49

$4.6 \quad L / l y$ als Funktion von $R a_{c}$ und $R a_{T} \ldots \ldots \ldots \ldots$. . . . . . . . 51 
$4.7 S h$ als Funktion von $R a_{c}$ über die gesamte Treppe . . . . . . . . . . 52

$4.8 I$ als Funktion von $R e$ und $d / L \ldots \ldots \ldots \ldots \ldots$. . . . . . . . . . . 53

4.9 Überprüfung von $d / L \sim R a_{c}^{1 / 9}\left|R a_{T}\right|^{-1 / 3}$ in thermohalinen Treppen . . . . 55

4.10 Überprüfung von $R e \sim R a_{c}\left|R a_{T}\right|^{-1 / 2}$ in thermohalinen Treppen . . . . . . 56

4.11 Überprüfung von $S h \sim R a_{c}^{4 / 9}\left|R a_{T}\right|^{-1 / 12}$ in thermohalinen Treppen . . . . 56

$4.124 / 3$ Gesetz für die thermohalinen Treppen . . . . . . . . . . . . . . . 57

5.1 neuer Turnerwinkel . . . . . . . . . . . . . . . . . . . . . . 60

5.2 alle Messdaten im $R a_{c}-R a_{T}$-Phasenraum . . . . . . . . . . . . 63

7.1 $R e_{x}$ und $R e_{y}$ gegen $R a_{c}$ am Übergang aufgetragen für $R a_{c}=1 \cdot 10^{9}$ und

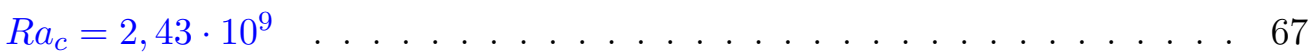

$7.2 R e_{x}$ und $R e_{y}$ gegen $R a_{c}$ am Übergang aufgetragen für $R a_{c}=1,73 \cdot 10^{10}$

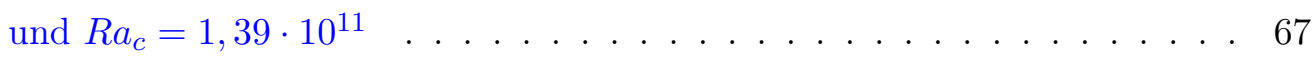

$7.3 R e_{x}$ und $R e_{y}$ gegen $R a_{c}$ am Übergang aufgetragen für $R a_{c}=1,85 \cdot 10^{11}$

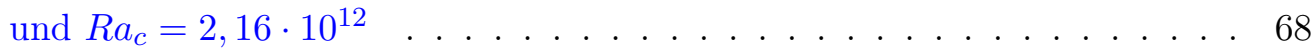

7.4 Sherwoodzahl gegen $R a_{T}$ für $R a_{c}=1 \cdot 10^{9}$ und $R a_{c}=1,73 \cdot 10^{10} \ldots$. . . 68

7.5 Sherwoodzahl gegen $R a_{T}$ für $R a_{c}=2,92 \cdot 10^{10}$ und $R a_{c}=1,39 \cdot 10^{11}$. . . 68

7.6 Sherwoodzahl gegen $R a_{T}$ für $R a_{c}=1,85 \cdot 10^{11}$ und $R a_{c}=2,16 \cdot 10^{12} \ldots$. 69

7.7 Reynoldszahl gegen $R a_{T}$ für $R a_{c}=1 \cdot 10^{9}$ und $R a_{c}=1,73 \cdot 10^{10} \ldots$. . . 69

7.8 Reynoldszahl gegen $R a_{T}$ für $R a_{c}=2,92 \cdot 10^{10}$ und $R a_{c}=1,39 \cdot 10^{11} \ldots$. . 69

7.9 Reynoldszahl gegen $R a_{T}$ für $R a_{c}=1,85 \cdot 10^{11}$ und $R a_{c}=2,16 \cdot 10^{12} \ldots 70$

7.10 Kalibrierkurven der Thermistoren . . . . . . . . . . . . . . . . . . . 80 


\section{Tabellenverzeichnis}

$2.1 \quad 4 / 3-G e s e t z \ldots \ldots \ldots \ldots \ldots 11$

3.1 Grenzströme der Elektrolytzelle . . . . . . . . . . . . . . . . . . . 22

3.2 Messdaten direkt am Übergang . . . . . . . . . . . . . . . . . . . . 35

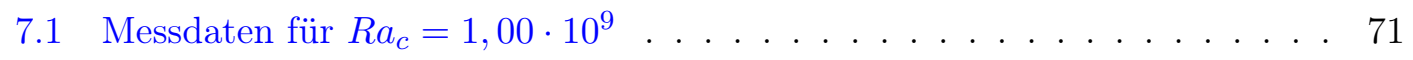

7.2 Messdaten für $R a_{c}=2,43 \cdot 10^{9} \ldots \ldots \ldots \ldots \ldots$. . . . . . . . . 72

7.3 Messdaten für $R a_{c}=1,73 \cdot 10^{10} \ldots \ldots \ldots \ldots \ldots \ldots$. . . . . . . . 72

7.4 Messdaten für $R a_{c}=2,92 \cdot 10^{10} \ldots \ldots \ldots \ldots \ldots \ldots$. . . . . . . . . . . . . . . . . . . 74

7.5 Messdaten für $R a_{c}=1,39 \cdot 10^{11} \ldots \ldots \ldots \ldots \ldots$. . . . . . . . 74

7.6 Messdaten für $R a_{c}=1,85 \cdot 10^{11} \ldots \ldots \ldots \ldots \ldots \ldots$

7.7 Messdaten für $R a_{c}=2,16 \cdot 10^{12} \ldots \ldots \ldots \ldots \ldots$

7.8 Messdaten für die Messungen zur thermohalinen Treppe für $|\Lambda| \approx 0,5 \quad$. . 76

7.9 Messdaten für die Messungen zur thermohalinen Treppe $|\Lambda| \approx 1,1 \ldots$. . . 77

7.10 Daten der verwendeten Thermostate . . . . . . . . . . . . . . . 79

7.11 Daten des verwendeten Multimeters . . . . . . . . . . . . . . . 81

7.12 Theoretische Daten zu den verwendeten Thermokristallen . . . . . . . . . 81

7.13 Daten des verwendeten Lasers . . . . . . . . . . . . . . . . . . . . 82

7.14 Daten der verwendeten Kamera . . . . . . . . . . . . . . . . . . . 83

7.15 Daten der verwendeten Tracerpartikel . . . . . . . . . . . . . . . . . 84 


\section{Einleitung}

Mitte des 20. Jahrhunderts stellten [Stommel et al., 1956] fest, dass, wenn man ein langes Metallrohr senkrecht im tropischen Ozean ins Wasser hält und oben etwas Meerwasser aus dem Rohr abpumpt, die Bewegung des Meerwassers (Aufwärtsbewegung in dem Rohr) selbst nach Abstellen der Pumpen erhalten bleibt. Dies lässt sich darauf zurückführen, dass in diesen Meeresregionen das Oberflächenwasser wärmer und salzhaltiger ist als das Tiefenwasser. Pumpt man nun etwas Wasser in einem Rohr nach oben, so kann sich die Temperatur mit dem Wasser außerhalb des Rohres ausgleichen, die Salzkonzentration aber nicht. So wird das aufsteigende Wasser immer leichter. Analog geschieht das Gleiche bei absinkendem Wasser in der Röhre. [Stern, 1960] stellte fest, dass diese Bewegungen auch ohne das Rohr stattfinden können und auf natürliche Weise in diesen Gewässern zu finden sind. Diese neue Art der Konvektion wurde Fingerströmung nach ihren charakteristischen schmalen Konvektionssäulen benannt. Er postulierte auch ein diffusives oszillatorisches Regime mit umgekehrter Stratifizierung. Diese beiden waren allerdings nicht die Ersten, die dieses Phänomen entdeckten. [Jevons, 1857] und [Rayleigh, 1883] entdeckten Fingerströmung, erkannten jedoch nicht die physikalischen Hintergründe. Ekman entdeckte 1906 bei einem Experiment mit Milch und Wasser Fingerströmung und erkannte auch das physikalische Prinzip dahinter, schätzte es aber als Kuriosum ein. Er glaubte nicht, dass auch andere Systeme dieser Art diese Strömung hervorrufen können.

Für doppelt diffusive Strömung sind in einem Arbeitsfluid mindestens zwei gelöste Komponenten nötig. Beide Komponenten müssen die Dichte des Arbeitsfluides beeinflussen. Wichtig hierbei ist vor allem, dass ihre Diffusivitäten, d.h. die Geschwindigkeit der Diffusion dieser Komponenten im Arbeitsfluid, verschieden sind. In etwa 30\% des Ozeanvolumens kann Fingerströmung auftreten. Etwa 15\% liegen im Parameterbereich des diffusiven oszillatorischen Regimes (Vgl. [You, 2002]). Somit kann fast die Hälfte des Ozeans mit doppelt diffusiver Strömung beschrieben werden. Dabei kann gerade Fingerströmung effektiv Salz oder andere gelöste Stoffe vom oberen Ozean in die tieferen Bereiche transportieren und sollte damit einen wichtigen Anteil an der Klimaentstehung auf der Erde 


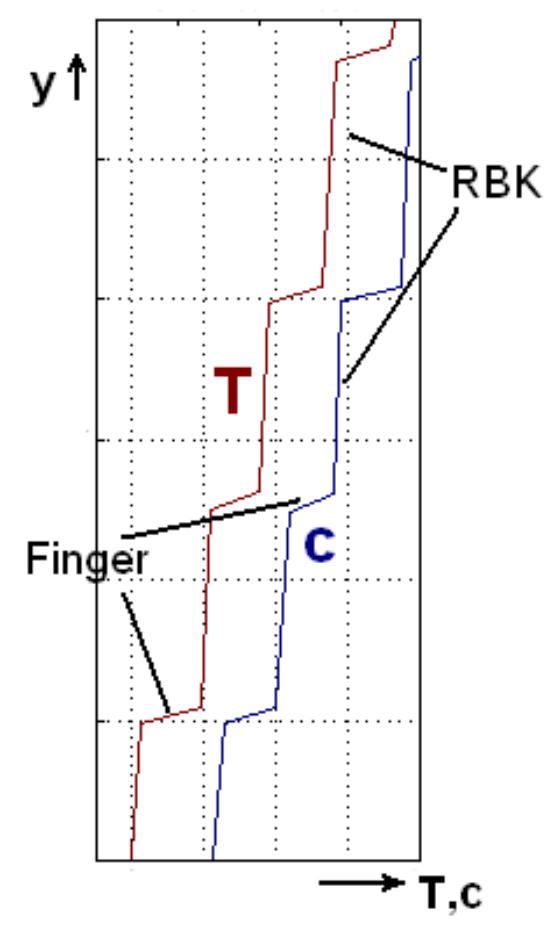

ABB. 1.1: Schematisches T-S-Profil einer thermohalinen Treppe im Fingerregime aus abwechselnden Bereichen mit Fingerströmung und Rayleigh-Bénard-Konvektion (RBK).

haben.

Dazu kommen noch weitere Systeme wie Sterne, Gasriesen, Magmakammern, Grundwasserquellen oder sogenannte Solar Ponds. Eine Übersicht darüber liefert unter anderem das Buch Double-Diffusive Convektion ([Radko, 2013]).

Schon [Stern, 1969] hat festgestellt, dass die Finger in der Fingerströmung nicht beliebig lang werden. In seinem Paper postuliert er, dass dafür interne Wellen verantwortlich sind, die umso stärker werden, je länger die Finger werden. Ab einer gewissen Länge wachsen die Finger nicht weiter, treiben dafür aber Rayleigh-Bénard-Konvektion an. Diese hat aber auch nur eine endliche Tiefe, bevor sich wieder ein Bereich mit Fingern bildet. Da dadurch ein Temperaturverlauf wie in Abbildung 1.1 entsteht, nennt man dieses Phänomen thermohaline Treppen. Diese wurden auch in den 60er Jahren im Ozean entdeckt ([Tait and Howe, 1968] oder [Cooper and Stommel, 1968]). Deren genaue Entstehung und physikalische Eigenschaften werden seitdem intensiv erforscht. Allerdings konnten bis jetzt keine großen Fortschritte gemacht werden. 
Die Arbeiten von [Hage and Tilgner, 2010] und [Yang et al., 2015b] zeigen, dass es nötig ist, die Eigenschaften der Fingerströmung nahe des Überganges zur Konvektion nocheinmal näher zu betrachten und vor allem im Parameterraum genau zu lokalisieren. Auch die Eigenschaften bzw. die Existenz thermohaliner Treppen nahe des Überganges zur Konvektion müssen noch untersucht bzw. nachgewiesen werden. Diese beiden großen Themenbereiche werden in dieser Arbeit experimentell untersucht.

\section{Gliederung}

In Kapitel 2 werden zuerst die physikalischen Hintergründe zur doppelt diffusiven Konvektion beschrieben. Dabei werden die Gleichungen, Randbedingungen und dimensionslosen Parameter eingeführt. Zudem wird in Abschnitt 2.1.3 und 2.2 kurz auf besondere Strömungsmuster eingegangen, die für diese Arbeit wichtig sind. Die Funktionsweise der Messapparatur und die Messverfahren werden hier auch vorgestellt.

In Kapitel 3 wird auf den experimentellen Aufbau für die Messungen "Grenzen des Fingerregimes" eingegangen (Abschnitt 3.2), bevor die Ergebnisse der Versuchsreihen im Abschnitt 3.3 vorgestellt und diskutiert werden.

In Kapitel 4 werden zuerst die Unterschiede der zweiten Versuchsreihe im Versuchsaufbau zu Kapitel 3 dargestellt, bevor im Abschnitt 4.3 die Ergebnisse zu den thermohalinen Treppen vorgestellt und diskutiert werden.

Abschließend gibt es in Kapitel 5 eine Zusammenfassung der Ergebnisse dieser Arbeit. 



\section{Theoretische Grundüberlegungen}

\subsection{Doppelt diffusive Strömung}

\subsubsection{Grundgleichungen und Kennzahlen}

Für die Beschreibung eines einfachen doppelt diffusiven Regimes sind vier Gleichungen notwendig. Mit T werden alle Größen bezeichnet, die zur schneller diffundierenden Komponente gehören. In dieser Arbeit ist dies die Wärme. Mit $c$ werden alle Größen gekennzeichnet, die zur langsamer diffundierenden Komponente gehören. In dieser Arbeit ist dies die Kupferionenkonzentration.

Die Navier-Stokes-Gleichung beschreibt den Transport eines Flüssigkeitsvolumens der Dichte $\rho$.

$$
\frac{\delta \vec{v}}{\delta t}+\vec{v}(\vec{\nabla}) \vec{v}=-\frac{1}{\varrho} \vec{\nabla} p+\nu \vec{\nabla}^{2} \vec{v}+\vec{F}
$$

Dabei ist $\vec{v}$ die Geschwindigkeit, $p$ der Druck und $\nu$ die kinematische Viskosität. $\vec{F}$ beschreibt alle von Extern auf das System wirkenden Kräfte. In dieser Arbeit besteht $\vec{F}$ somit nur aus der Auftriebskraft $\vec{F}=\vec{g} \rho_{0}^{-1} \Delta \rho$. Wobei das $\Delta \rho$ durch die Konzentration und die Temperatur beeinflusst wird.

Die Kontinuitätsgleichung beschreibt den Erhalt der Masse in einem Fluidvolumen.

$$
\frac{\delta \rho}{\delta t}+\vec{\nabla}(\rho \vec{v})=0
$$

Im Fall von inkompressibler Strömung vereinfacht sie sich zu $\vec{\nabla} \vec{v}=0$.

Die Wärmetransportgleichung beschreibt den Wärmetransport analog zur Massenerhaltung.

$$
\frac{\delta T}{\delta t}+\vec{v} \vec{\nabla} T=\vec{\nabla}\left(\kappa_{T} \vec{\nabla} T\right)
$$

Eine weitere Gleichung ist noch nötig, um die Änderung der Konzentration zu beschreiben. Diese Gleichung wird als Nernst-Plank-Gleichung bezeichnet.

$$
\frac{\delta c}{\delta t}+\vec{v} \vec{\nabla} c=\vec{\nabla}\left(\kappa_{c} \vec{\nabla} c\right)
$$


$\kappa_{T}$ und $\kappa_{c}$ sind die Diffusivitäten der Komponenten.

Durch Entdimensionalisierung dieser vier Grundgleichungen lassen sich vier dimensionslose Kennzahlen finden, die das System eindeutig beschreiben:

$$
\begin{aligned}
R a_{T} & =\frac{g \alpha_{T} \Delta T l_{y}^{3}}{\kappa_{T} \nu} \\
R a_{c} & =\frac{g \alpha_{c} \Delta c l_{y}^{3}}{\kappa_{c} \nu} \\
S c & =\nu / \kappa_{c} \\
P r & =\nu / \kappa_{T}
\end{aligned}
$$

Dabei ist $g$ der Betrag der Erdbeschleunigung, $l_{y}$ die Zellhöhe, $\alpha_{c}$ und $\alpha_{T}$ sind die Koeffizienten der Dichtevariation mit der Konzentration bzw. Temperatur. Aus technischen Gründen sind in dieser Arbeit $\operatorname{Pr}$ und $S c$ annähernd konstant ${ }^{1}$. Daher bleiben nur die beiden Rayleighzahlen als variable Parameter übrig. Zu beachten ist, dass die chemische Rayleighzahl mit $\kappa_{c}$ gebildet wird und nicht wie häufig in der Literatur mit $\kappa_{T}$.

Die Rayleighzahlen sind ein Maß für die Auftriebskraft, analog zur Rayleigh-BénardKonvektion. Jedoch können in dieser Arbeit die Rayleighzahlen auch negativ werden. Dies ergibt sich aus der Definition von $\Delta T=T_{\text {unten }}-T_{\text {oben }}$ bzw. $\Delta c=c_{\text {oben }}-c_{\text {unten }}$ und bedeutet, dass die Schichtung des Fluides bezüglich der Komponente stabil ist.

Schmidt- und Prandtlzahl sind Fluideigenschaften und beschreiben das Verhältnis der Diffusivitäten von Impuls zu Konzentration bzw. Temperatur. Der Quotient aus beiden ist die Lewiszahl: $L e=S c / P r=\kappa_{T} / \kappa_{c}$. Sie beschreibt das Verhältnis der Diffusivitäten der Komponenten. In der Literatur wird oft mit dem Kehrwert der Lewiszahl $\tau$ gerechnet.

Eine weitere wichtige Kennzahl ist das Auftriebsverhältnis $\Lambda$. Es beschreibt, ob die Dichteschichtung stabil oder instabil ist.

$$
\Lambda=\frac{\alpha_{T} \Delta_{T}}{\alpha_{c} \Delta_{c}}=L e R a_{T} R a_{c}^{-1}
$$

Im Fingerregime, welches Kern dieser Arbeit ist, ist $\Lambda$ negativ, da die thermische Rayleighzahl negativ und die chemische positiv ist. Daher wird in dieser Arbeit nur der Betrag vom Auftriebsverhältnis betrachtet. Es folgt daher für $|\Lambda|<1$ eine instabile Schichtung des Fluides und für $|\Lambda|>1$ eine stabile Schichtung des Fluides. Bei $|\Lambda|=0$ verschwindet der Temperaturgradient und es liegt einfach chemisch getriebene RayleighBénard-Konvektion vor.

\footnotetext{
${ }^{1} 8,55<\operatorname{Pr}<8,99$ und $1910<S c<2114$
} 
Zwei weitere Kennzahlen sind für diese Arbeit noch von Bedeutung. Dies sind die Reynoldszahl und die Sherwoodzahl.

$$
\begin{aligned}
R e & =\frac{v l_{y}}{\nu} \\
S h & =\frac{j\left(1-t_{i}\right) l_{y}}{z F \Delta c \kappa_{c}}
\end{aligned}
$$

mit $z$ als Wertigkeit der Kupferionen, $F$ der Faraday-Konstante, $j$ der Stromdichte und $t_{i}$ der Transportzahl. Die Reynoldszahl setzt Trägheitskraft und Reibungskraft ins Verhältnis. Ist diese groß genug, kann Turbulenz im System auftreten.

Die Sherwoodzahl setzt den gesamten Massentransport ins Verhältnis zum diffusiven Massentransport. Da es in dieser Arbeit bei den transportierten Teilchen um geladene Kupferionen geht, muss noch berücksichtigt werden, dass diese auch durch elektrische Felder bewegt werden können (vgl. Kapitel 2.3). Dies wird über die Transportzahl realisiert. Der Einfluss dieser auf den Strom liegt in der Größenordnung $10^{-3}$, also im Promillebereich.

\subsubsection{Randbedingungen}

Bei der Betrachtung eines Systems sind die Randbedingungen extrem wichtig, da sie die Strömung beeinflussen. Für alle Ränder gilt die Haftbedingung, d.h. die Geschwindigkeiten direkt am Rand sind 0. An den Seitenrändern wird angenommen, dass diese thermisch, chemisch und elektrisch neutral sind. An der oberen und unteren Platte hingegen gibt es eine feste Konzentration und eine feste Temperatur.

$$
\begin{array}{r}
\text { oben: } \mathrm{c}=\mathrm{c}_{0}+\Delta_{\mathrm{c}} / 2 \text { und } \mathrm{T}=\mathrm{T}_{0}-\Delta \mathrm{T} / 2 \\
\text { unten : } \mathrm{c}=\mathrm{c}_{0}-\Delta_{\mathrm{c}} / 2 \text { und } \mathrm{T}=\mathrm{T}_{0}+\Delta \mathrm{T} / 2
\end{array}
$$

Zuletzt gilt noch die Elektronenneutralität, d.h. die Summe der Ladungen aller geladenen Teilchen ist Null.

$$
\sum z_{i} c_{i}=0
$$

Der Index $i$ kennzeichnet die verschiedenen Ionensorten, die im Fluid gelöst sind. Nahe der Platten in der Nernstschen Diffusionsschicht herrscht Ladungstrennung. Allerdings ist diese nur einige Nanometer groß und wird daher vernachlässigt. 


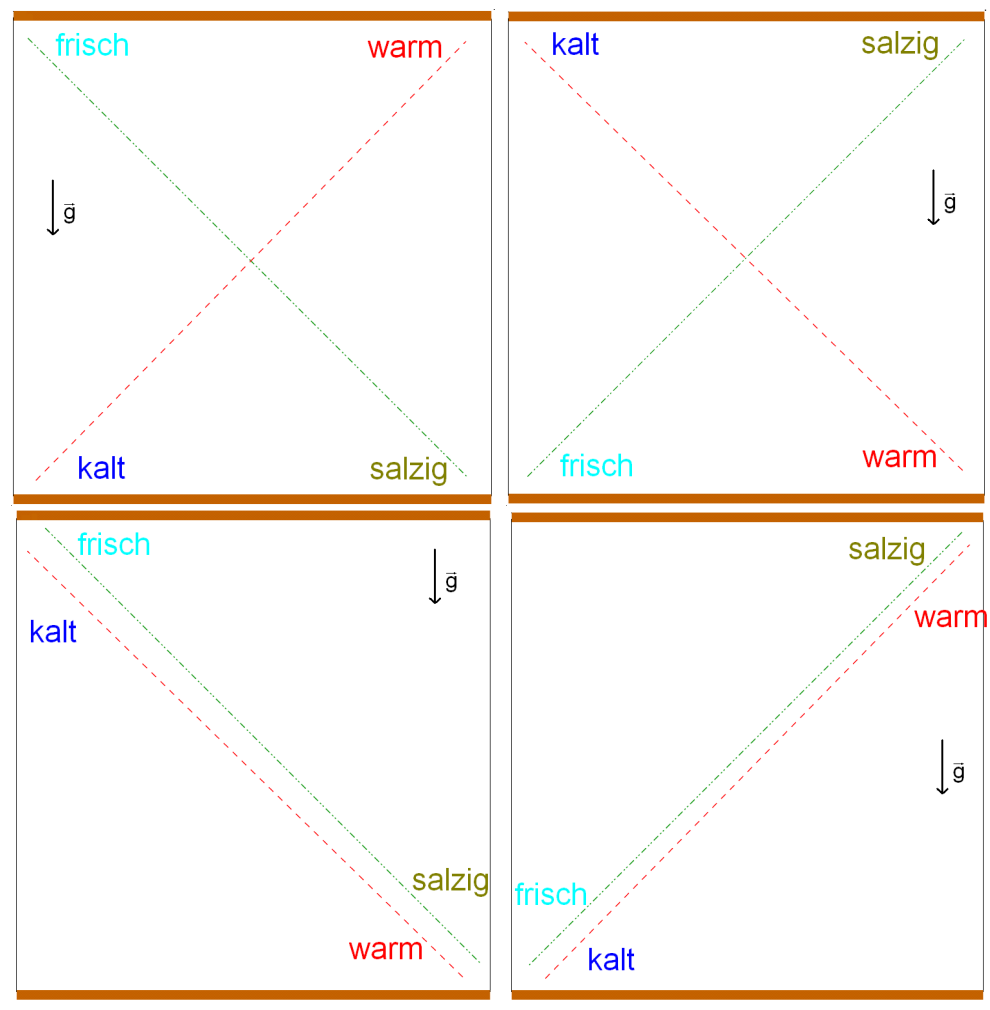

ABB. 2.1: Regime in doppelt diffusiver Strömung. OBEN LINKS $\rightarrow$ doppelt stabiles Regime, OBEN RECHTS $\rightarrow$ doppelt instabiles Regime, UNTEN LINKS $\rightarrow$ diffusives Regime, UNTEN RECHTS $\rightarrow$ Fingerregime.

\subsubsection{Regime der doppelt diffusiven Konvektion}

Doppelt diffusive Strömung taucht dann auf, wenn zwei Stoffe ${ }^{2}$ mit unterschiedlichen Diffusionszeiten die Dichte des Mediums ändern. Dadurch können vier Konfigurationen entstehen. Beide können stabil geschichtet sein (Abb. 2.1 oben links). In diesem Fall herrscht Diffusion vor und es kommt zu keiner Strömung. Sind beide instabil geschichtet, dann herrscht Rayleigh-Bénard-Konvektion vor (Abb. 2.1 oben rechts). Interessant werden erst die beiden übrigen Konfigurationen. Ist die schneller diffundierende Komponente instabil und die langsamere stabil geschichtet, liegt das diffusive Regime vor (Abb. 2.1 unten links). Hier kann es zu oszillatorischen Instabilitäten kommen, die zu einem Wechsel von Konvektionsbereichen und dünnen Grenzbereichen führen.

Für diese Arbeit steht allerdings das Fingerregime im Mittelpunkt (Abb. 2.1 unten rechts). Hier ist die schneller diffundierende Komponente stabil und die langsam diffun-

\footnotetext{
${ }^{2}$ Zur Vereinfachung wird die Temperatur hier auch als Stoff bezeichnet.
} 


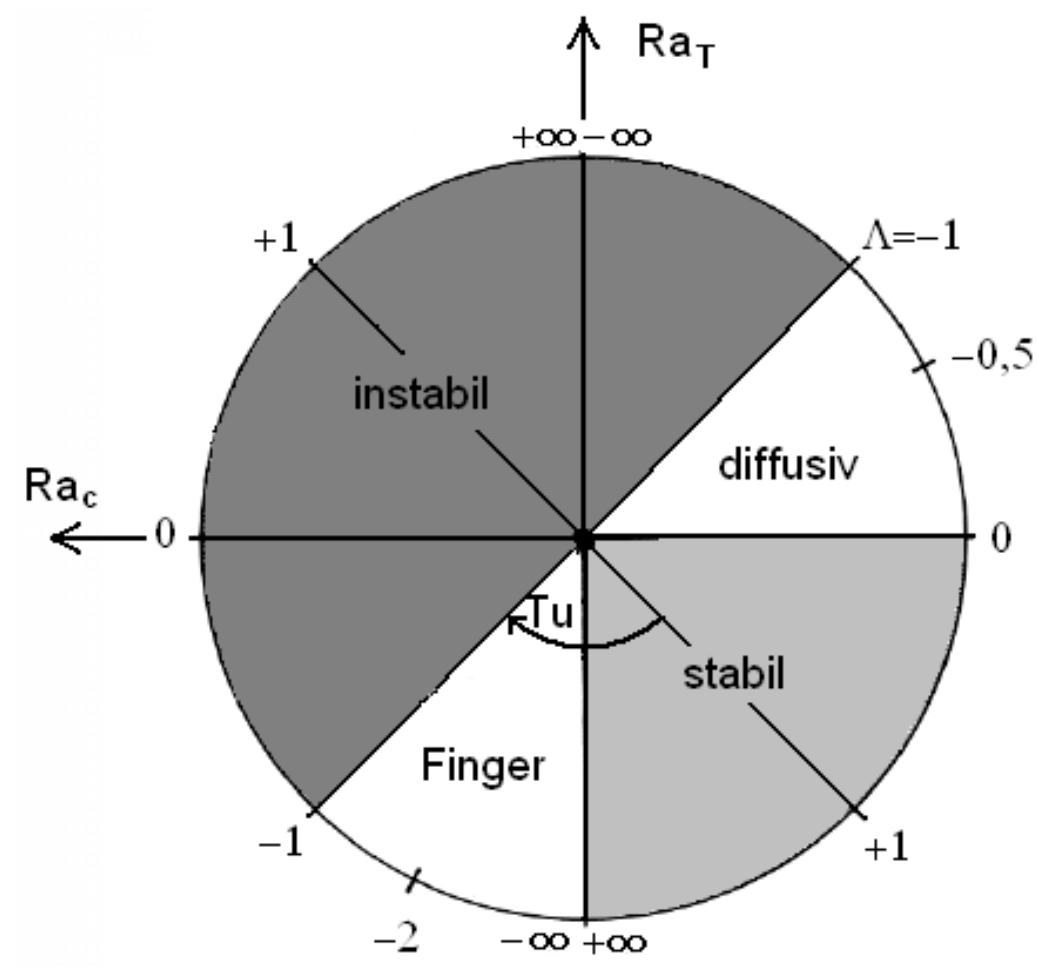

AвB. 2.2: Schematische Darstellung der doppelt diffusiven Regime über den Turnerwinkel (vgl. [Ruddick, 1983]).

dierende instabil geschichtet. Selbst wenn der globale Gradient der Dichte stabil ist, kann es zu Konvektion kommen. Ein ausgelenktes Fluidpartikel gleicht schnell seine Temperatur mit der Umgebung aus, die Salzkonzentration bleibt jedoch fast unverändert. Daher sinkt bzw. steigt er weiter. Dies führt zu einem linearen Temperaturprofil. Das Konzentrationsprofil weist hingegen starke horizontale Gradienten zwischen absinkendem und aufsteigendem Fluid auf.

Daher ergibt sich im $R a_{T}-R a_{c}$-Phasenraum eine Unterteilung in vier Gebiete (vgl. Abb. 2.2). Diese können über den Turnerwinkel dargestellt werden. Dieser ist definiert als:

$$
T u=135^{\circ}-\arg \left(-\alpha_{c} \Delta c+i \alpha_{T} \Delta T\right)
$$

Dadurch ergeben sich für das Fingerregime der Bereich $45^{\circ}<T u<90^{\circ 3}$ und für das diffusive Regime $-90^{\circ}<T u<-45^{\circ}$.

\footnotetext{
${ }^{3}$ Genau genommen ist die untere Grenze nicht bei $45^{\circ}$, da Fingerströmung abhängig vom System nur im Bereich $>45^{\circ}$ entstehen kann. Bei Wärme-Salz ist die Grenze z.B. $\approx 45,7^{\circ}<T u<90^{\circ}$. Vgl. hierfür Kapitel 3.1.
} 


\subsection{Thermohaline Treppen}

In diesem Kapitel werden die Eigenschaften von thermohalinen Treppen erläutert. Ihre Entstehung wird in Kapitel 4.1 näher betrachtet.

Eine besondere Form der doppelt diffusiven Konvektion bilden die thermohalinen Treppen. Diese können im diffusiven als auch im Fingerregime auftauchen. Da für diese Arbeit nur die Treppen im Fingerregime von Bedeutung sind, wird die Betrachtung der Treppen im diffusiven Regime hier vernachlässigt.

Schon kurz nach der Entdeckung der doppelt diffusiven Strömung wurden auch die thermohalinen Treppen entdeckt ([Tait and Howe, 1968] und [Cooper and Stommel, 1968]). Diese bestehen aus einer Folge von Fingern und Konvektionswalzen in vertikaler Richtung. Dabei können die Finger eine Länge von einigen Dezimetern und eine Breite von einigen Zentimetern im tropischen Ozean erreichen. Die Konvektionswalzen haben dann eine Ausdehnung von einigen dutzend Metern. Die ganze Abfolge von Fingern und Walzen kann sich einige Kilometer in die Tiefe erstrecken und über 100 Kilometer in horizontaler Richtung (vgl. u.a. [Schmitt et al., 1987]). Dabei können sie über Jahrzehnte stabil bleiben. Auch im Labor (vgl. u.a. [Turner, 1967], [Stern and Turner, 1969] oder [Krishnamurti, 2003]) oder numerisch (vgl. u.a. [Stellmach et al., 2011]) wurden solche Treppen entdeckt.

Thermohaline Treppen durchlaufen während ihrer Lebensdauer von mehreren Jahrzehnten auch einige Veränderungen. [Zodiatis and Gasparini, 1996] haben im Mittelmeer thermohaline Treppen über einen Zeitraum von fast 20 Jahren untersucht. Dabei stellten sie fest, dass die Treppen ortsgebunden sind, d.h. es gibt kaum einen vertikalen oder horizontalen Drift der gesamten Treppe. Die Anzahl der Stufen nahm jedoch in den 20 Jahren von neun auf fünf ab. Dabei verschmolzen Konvektionsbereiche zu größeren Konvektionsbereichen, indem sie die Fingerbereiche dazwischen vereinnahmten.

Ein umgekehrter Prozess kann im Labor beobachtet werden ([Krishnamurti, 2003]). Während eines Run-Down-Experiments, d.h. die Randbedingungen für $T$ und $S$ werden nicht konstant gehalten, wuchsen die Fingerregime und vereinnahmten die Konvektionsbereiche, bis nur ein großes Fingerregime übrig blieb. Im selben Experiment konnte man durch Konstanthalten der Randbedingungen für $T$ und $S$ die Anzahl der Treppenstufen konstant halten. Thermohaline Treppen wurden bis jetzt nur in Bereichen mit niedrigen Auftriebsverhältnissen $(1<|\Lambda|<2$ im Salz-Wärme-Fall) entdeckt. Dabei steigt die Stufen- 
höhe mit abnehmendem Auftriebsverhältnis.

Von Interesse für die Wissenschaft sind bei den thermohalinen Treppen vor allem der Transport von Masse und Temperatur. Für einem einzelnen Fingerbereich in einer thermohalinen Treppe (Fingerinterface) hat [Turner, 1965, Turner, 1967] eine Abhängigkeit von

$$
j \sim f(\Lambda, L e, \operatorname{Pr}) \Delta c^{4 / 3}
$$

das sogenannte 4/3-Gesetz, gefunden. Eine analoge Form des 4/3 Gesetzes gibt es auch für den Temperaturfluss.

$$
Q \sim f(\Lambda, L e, \operatorname{Pr}) \Delta T^{4 / 3}
$$

Um das 4/3-Gesetz mit Gleichung 3.6 zu vergleichen kann man Gleichung 2.13 auch wie folgt schreiben:

$$
j / \Delta c \sim f(\Lambda, L e, \operatorname{Pr}) \Delta c^{1 / 3} \Rightarrow S h \sim f(\Lambda, L e, \operatorname{Pr}) R a_{c}^{1 / 3}
$$

Allerdings wurden hierbei die Größe der Konvektionsbereiche und die Startbedingungen vernachlässigt. Neuere Studien haben daher kleinere Korrekturen zum 4/3-Gesetz gefunden. Diese sind in Tabelle 2.1 zusammengefasst.

Die Sherwoodzahl und damit der Salzfluss ist zusätzlich noch abhängig von der Anzahl der Interfaces $n$ und hat sein Maximum, wenn nur Finger vorhanden sind. Danach fällt Sh mit ca. $1 / n$ ab (vgl. [Krishnamurti, 2003]).

\begin{tabular}{|c|c|c|}
\hline Modell/Experiment & Exponent $p$ & Basis \\
\hline [Schmitt, 1979a] & $1,24-1,37$ & experimentell \\
[McDougall and Taylor, 1984] & 1,23 & experimentell \\
[Krishnamurti, 2003] & $1,18-1,19$ & experimentell \\
[Hage and Tilgner, 2010] & $4 / 3$ & experimentell \\
[Özgökmen et al., 1998] & $4 / 3$ & numerisch \\
[Paparella, 2000] & $1,33-2,0$ & numerisch \\
[Sreenivas et al., 2009] & 1,32 & numerisch \\
\hline
\end{tabular}

ТАВ. 2.1: Überprüfung des 4/3-Gesetzes auf Basis von Fits der Form $j \sim(\Delta c)^{p}$ an die Datenpunkte der jeweiligen Arbeiten. 


\subsection{Funktionsweise der elektrochemischen Zelle}

Um die Dichte des Fluides zu ändern, wird zum einen eine Temperaturdifferenz angelegt, zum anderen eine Stoffkonzentrationsdifferenz erzeugt. In diesem Fall handelt es sich bei dem gelösten Stoff um Kupferionen. Dies geschieht über Elektrolyse.

In einem Arbeitsfluid (deionisiertes $\mathrm{H}_{2} \mathrm{O}$ ) ist Kupfersulfat gelöst. Dieses dissoziiert zu Kupferionen $\left(\mathrm{Cu}^{2+}\right)$ und Sulfationen $\left(\mathrm{SO}_{4}^{2-}\right)$. Die beiden Kupferplatten dienen als Elektroden. An diesen lösen sich Kupferionen in das Fluid und umgekehrt bis sich eine Gleichgewichtspotentialdifferenz zwischen Elektrolyt und Elektrode einstellt. Diese ist stoffspezifisch und beträgt in unserem Fall $C u / C u^{2+}: \phi_{o}=0,34 \mathrm{~V}$.

Durch Anlegen einer äußeren Spannung kommt es zur Störung des Gleichgewichts und an der Anode wird das Lösen der Kupferionen begünstigt, da hier ein Elektronenmangel vorliegt, und an der Kathode das Ablagern von Kupferionen, da hier ein Elektronenüberschuss vorherrscht. Dadurch kommt es zu folgenden Reaktionen:

$$
\begin{aligned}
& \text { an der Anode }: \mathrm{Cu} \rightarrow \mathrm{Cu}^{2+}+2 \mathrm{e}^{-} \\
& \text {an der Kathode }: \mathrm{Cu}^{2+}+2 \mathrm{e}^{-} \rightarrow \mathrm{Cu}
\end{aligned}
$$

Es entsteht eine Potentialdifferenz zwischen den Elektroden durch die angelegte Spannung, sodass ein Strom fließst. Je stärker die externe Spannung, desto mehr wird das System aus dem Gleichgewicht gedrängt, d.h. desto mehr Kupferionen können sich lösen bzw. anlagern.

\section{Transport der Kupferionen}

Durch das Anlegen einer externen Spannung kommt es an der Anode zu einer erhöhten Konzentration von Kupferionen im Arbeitsfluid. An der Kathode fehlen diese. Das System versucht diesen Unterschied durch Diffusion auszugleichen. Außerdem wirkt auf die geladenen Kupferionen das externe elektrische Feld, sodass sie von der Kathode angezogen werden. Dadurch entsteht ein Ionenfluss, der ebenfalls den Konzentrationsgradienten ausgleicht. Diese Art der Bewegung nennt man Migration. Außerdem ist es möglich, dass die Ionen eine instabile Dichteschichtung im Arbeitsfluid hervorrufen. Dadurch kommt es zur Konvektion, die ebenfalls die Konzentrationsdifferenz senkt.

Da in den betrachteten natürlichen Systemen die Migration, oder ein entsprechender Transportmechanismus, nicht vorkommt, muss diese unterbunden werden, um die Ergebnisse später übertragen zu können. Dies wird erreicht, indem im Arbeitsfluid eine 
starke Säure oder Base in hoher Konzentration gelöst wird. Diese dissoziiert vollständig. Dadurch entstehen geladene Teilchen (Leitsalz) im Arbeitsfluid, welche das elektrische Feld abschirmen, sodass die Kupferionen durch jenes keine Kraft mehr erfahren. Der Fehler, den die Migration verursacht, ist dann von der Größenordnung des Quotienten aus Kupferkonzentration zu Leitsalzkonzentration (vgl. [Levich, 1962]). Zudem zeigen numerische Simulationen von [Gerstung, 2010], dass die zusätzlich auftauchenden Ionen kaum eine Rolle spielen, wenn die Konzentration des Leitsalzes mindestens das zehnfache gegenüber der des Kupfersulfates erreicht.

\section{Kennlinie der elektrochemischen Zelle}

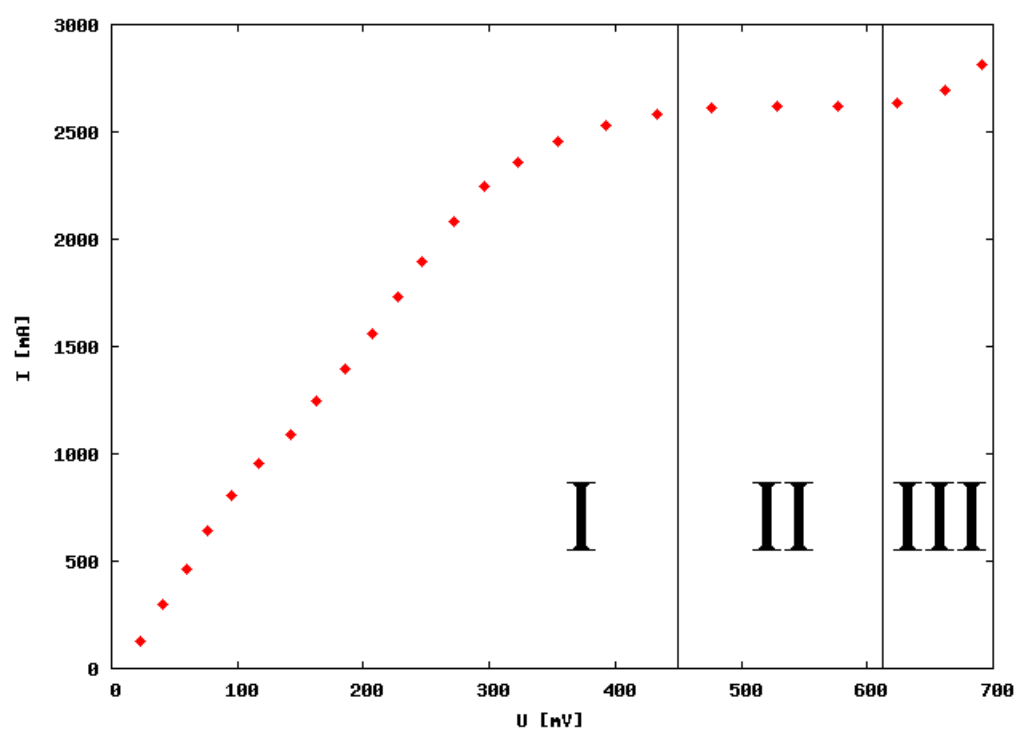

AвB. 2.3: Kennlinie der doppelt diffusiven Messzelle. Man erkennt den fast linearen Anstieg der Stromstärke bei kleinen Spannungen, das Plateau bei mittleren Spannungen (Grenzstrom) und einen weiteren Anstieg bei hohen Spannungen durch zusätzliche Reaktionen.

Misst man den Stromfluss durch die Zelle, so stellt man fest, dass dieser u.a. mit der Spannung variiert. Stellt man sich die Zelle und Elektroden als Stromkreis vor, so gibt es drei Widerstände in diesem. Der erste hängt von der Leitfähigkeit der Ionen im Elektrolyt ab und ist ionenspezifisch und von der Stromstärke unabhängig, d.h. ohmisch. Die anderen Widerstände erfahren die Ionen direkt an den Elektroden und sind der spezifische Widerstand der Anode und Kathode. Diese sind von der Stromstärke abhängig.

Die von außen angelegte Spannung setzt sich aus der Überspannung $\eta$, den Ruhepoten- 
tialen $\phi_{0}^{a}, \phi_{0}^{b}$ an den Elektroden ${ }^{4}$ und dem Spannungsabfall am Elektrolyt $\Delta U$ zusammen.

$$
U=\eta+\Delta U+\phi_{0}^{a}-\phi_{0}^{b}=\eta+\Delta U
$$

Die Überspannung hängt von mehreren Faktoren ab (vgl. umfassend

[C.H.Hamann and W.Vielstich, 1980]). Dadurch kommt es zu der in Abbildung 2.3 typischen Kennlinie. Diese lässt sich in drei Bereiche einteilen:

Bereich I: Die Reaktionen 2.16 und 2.17 verlaufen an den jeweiligen Elektroden mit endlicher Geschwindigkeit, welche wesentlich von der angelegten Spannung abhängt. Die Überkonzentration der Kupferionen an der Anode und deren Mangel an der Kathode wird durch Diffusion hinreichend schnell ausgeglichen, sofern die Spannung klein genug ist. Es bildet sich ein lineares Kupferionenkonzentrationsprofil. Steigt die Spannung, reagieren mehr Kupferionen an den Elektroden und der Strom steigt. Ist der Abtransport der Kupferionen durch Diffusion nicht mehr schnell genug, so werden die Reaktionen gehemmt. Dadurch kommt es zu Dichtevariationen und Konvektion, sobald die kritische Rayleighzahl überschritten wird. Daher wird immer sichergestellt, dass der Abtransport genügend schnell stattfindet. Jeder Spannung kann ein eindeutiger Strom zugeordnet werden.

Bereich II: Steigt die Spannung hoch genug, kann nicht jedes Kupferion, welches aus der Anode ausgelöst wird, sofort abtransportiert werden. Analog wird jedes Kupferion, welches die Kathode erreicht, sofort angelagert. In diesen Fall herrscht an der Kathode $c_{\text {Kathode }}=0$ und entsprechend $c_{\text {Anode }}=2 c_{0}$. Eine weitere Erhöhung der Spannung führt dann zu keiner Erhöhung des Stromes, da nicht mehr Kupferionen pro Zeit an der Kathode angelagert werden können. Dem entsprechend werden auch nicht mehr Kupferionen an der Anode freigesetzt. Es stellt sich ein Grenzstrom ein.

Bereich III: In diesem Bereich ist die Elektrodenspannung groß genug, um weitere Reaktionen zu ermöglichen. In diesem Fall findet die Elektrolyse von Wasser statt. Es entsteht an der Kathode unter Zugabe zusätzlicher Elektronen Wasserstoff und an der Anode unter Freisetzung zusätzlicher Elektronen Sauerstoff. Dies führt zu einer weiteren Erhöhung des Stromes.

$$
\begin{aligned}
\text { an der Anode: } & 6 \mathrm{H}_{2} \mathrm{O} \rightarrow \mathrm{O}_{2}+4 \mathrm{H}_{3} \mathrm{O}^{+}+4 e^{-} \\
\text {an der Kathode : } & 2 \mathrm{H}_{3} \mathrm{O}^{+}+2 e^{-} \rightarrow \mathrm{H}_{2}+2 \mathrm{H}_{2} \mathrm{O}
\end{aligned}
$$

Daher werden alle Messungen im Bereich II durchgeführt, da hier die Kupferkonzentra-

\footnotetext{
${ }^{4}$ Diese heben sich auf, da die Elektroden aus dem gleichen Material stammen
} 
tionen an den Platten bekannt sind und somit $\Delta c$ bestimmt werden kann, ohne aus der Zelle Fluid zu entnehmen.

\subsection{Messmethoden}
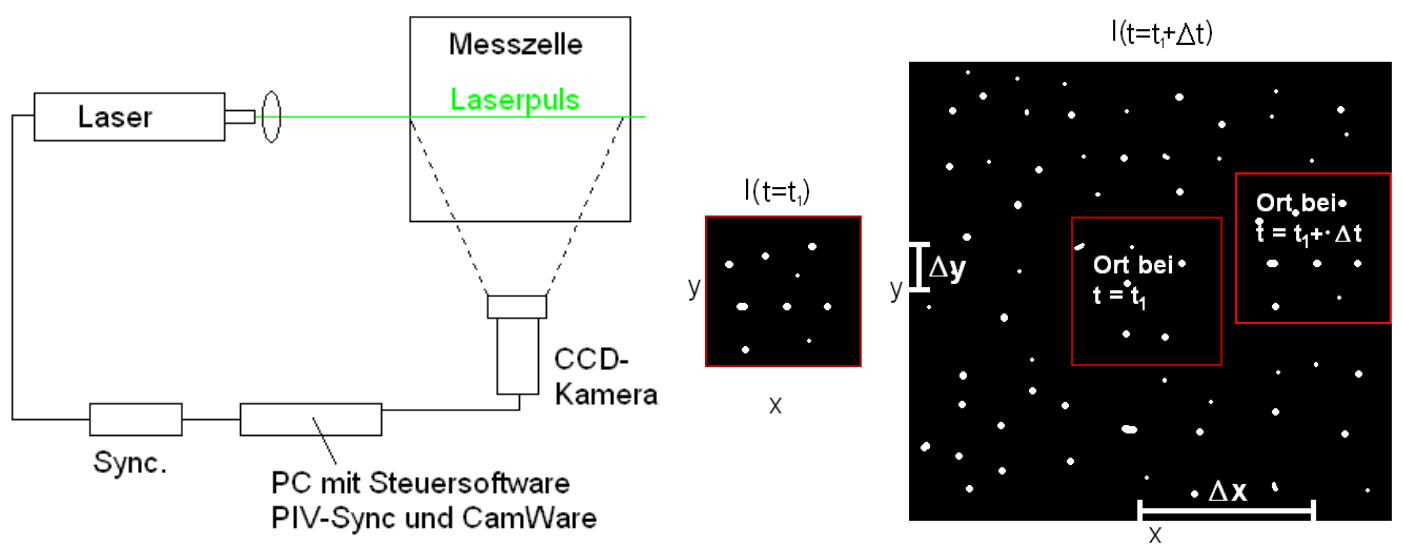

ABB. 2.4: LINKS: Schematischer Aufbau des PIV-Systems in dieser Arbeit.

RECHTS: Schematische Funktionsweise des PIV

\section{- Particle Image Velocemitry}

Das PIV-Verfahren ist ein nichtinvasives optisches Verfahren zur Bestimmung von Geschwindigkeitsfeldern. Dies ist in 2D und 3D möglich, wird in dieser Arbeit nur in 2D verwendet. In das Arbeitsfluid werden Tracerpartikel mit der Dichte des Fluides gefüllt. Dadurch werden sie von der Strömung mitgetrieben. Nun werden mittels einer Kamera zu 2 Zeitpunkten die Positionen der Partikel bestimmt. Es wird dann geschaut, in welche Richtung sich jeder Partikel bewegt hat und aus dem zeitlichen Abstand der Aufnahmen kann die Geschwindigkeit für jedes Partikel und damit auch für das Arbeitsfluid am Ort des Partikels bestimmt werden. Da es nicht so einfach ist, einen einzelnen Partikel zu verfolgen, wird bei der Auswertung immer eine Gruppe von Partikeln innerhalb eines Bildabschnittes genommen (vgl. Abbildung 2.4 (rechts)). Dabei wird dieser Bildabschnitt leicht verschoben und dann mit der neuen Position auf dem zweiten Bild verglichen. Dies geschieht über eine Kreuzkorrelation der Bildabschnitte. Als Maß für die Übereinstimmung wird der Korrelationskoeffizient genommen. Dabei treten natürlich Ausreißer auf. Diese werden durch Filtern (global ${ }^{5}$ und lokal) aussortiert und mittels Interpolati-

\footnotetext{
${ }^{5}$ Vgl. Abbildung 3.4
} 
on ersetzt. Dies geschieht in dieser Arbeit alles mit Hilfe des Programmes VidPIV 4.1. Zur Beleuchtung der Tracerpartikel wird ein Laserstrahl aufgefächert, sodass er eine 2D-Ebene beleuchtet. Das Streulicht der Tracerpartikel wird von der Kamera aufgenommen. Da die Genauigkeit des PiV-Verfahrens u.a. auch von der Aufnahmefrequenz abhängt, wurden bei jeder Messung mehrere Frequenzen wie bei [Hage, 2009] aufgenommen. Dadurch konnte immer gewährleistet werden, dass zu jeder Strömung die optimale Aufnahmefrequenz aufgenommen und somit der Fehler der PiV-Verfahrens minimiert wurde. Eine Zusammenfassung des PIV-Verfahrens ist u.a. in [Raffel et al., 1998, Grand, 1997] zu finden.

- Schattenverfahren (Shadowgraph)

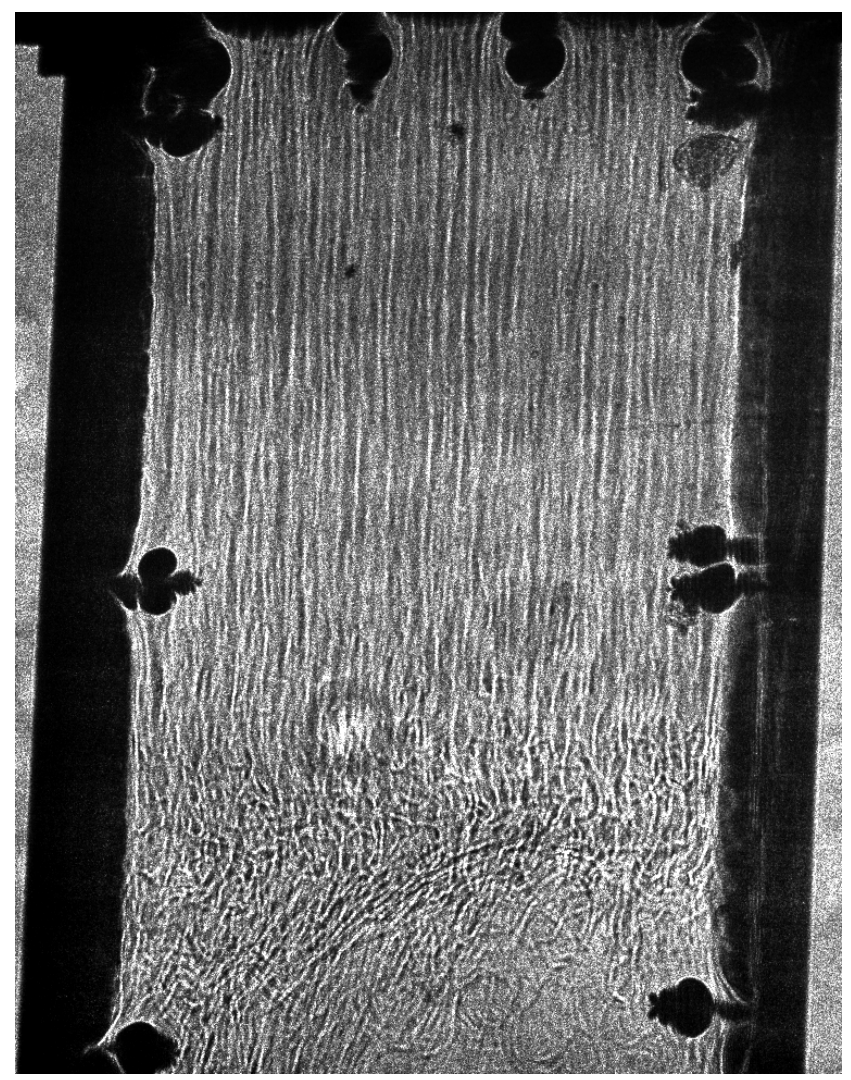

ABB. 2.5: Shadowgraphaufnahme der Zelle mit einer thermohalinen Treppe. Dargestellt ist eine Stufe. Im oberen Bereich sind die Finger deutlich zu erkennen und im unteren Bereich herrscht Rayleigh-Bénard-Konvektion vor. Das Bild erstreckt sich über einen 16,5 cm hohen Abschnitt der $80 \mathrm{~cm}$ hohen Zelle, sowie über die volle Zellbreite $(10 \mathrm{~cm})$. Die Parameter der Strömung sind die Gleichen wie in Abbildung 4.3. 
Auch das Schattenverfahren ist ein nichtinvasives Verfahren. Dabei wird ausgenutzt, dass das Fluid mit der Dichte auch seinen Brechungsindex ändert. Dies geschieht über die Gladstone-Dale-Beziehung.

$$
n-1=k \cdot \rho
$$

$\mathrm{k}$ ist dabei eine Konstante, die vom Druck der Wellenlänge des Lichtes, der Temperatur und dem Fluid selber abhängt. Dadurch entstehen auf einer Leinwand Interferenzmuster, wenn man die Zelle mit parallelen Lichtstrahlen durchleuchtet. Dies ist beispielhaft in Abbildung 2.5 zu sehen. Mit diesem Verfahren können relativ leicht die Strukturen der Strömung bestimmt werden, da vor allem im Fingerregime starke Dichtesprünge zwischen den Fingern existieren. Im konvektiven Bereich lassen sich vor allem Plumes mit diesem Verfahren identifizieren.

- Temperaturvisualisierung: Thermokristalle

Thermokristalle sind Stoffe, die ihre innere Struktur mit der Temperatur ändern, und dadurch Licht anderer Wellenlängen reflektieren. Es gibt Thermokristalle, die durchsichtig bis zu einer bestimmten Temperatur sind und dann Licht reflektieren. Andere Kristalle durchlaufen das gesamte Farbspektrum. Letztere Kristalle wurden in dieser Arbeit verwendet. Die genauen Daten der in dieser Arbeit verwendeten Thermokristalle befinden sich im Anhang (Kapitel 7.4). Die Zelle wurde zuerst mit den im Arbeitsfluid gelösten Kristallen gefüllt und dann eine Eichmessung bei linearem Temperaturgradienten gemacht. Dabei wird die Zelle von der Seite mit einer Lichtquelle beleuchtet und im rechten Winkel mit einer CCD-Kamera fotografiert. Danach wurde direkt die Spannung angeschaltet. Als das System im statistisch-stationären Regime war, wurden weitere Aufnahmen durchgeführt. Alle Aufnahmen lagen pixelweise im RGB-Farbraum vor. Diese wurden dann in den HSI-Farbraum (Farbton (hue), Sättigung (saturation), Intensität (intensity)) umgerechnet. Der H-Wert ist somit der einzige, der Informationen über den Farbton und damit über die Temperatur enthält (vgl. [Dabiri and Gharib, 1991]).

- Temperaturmessungen: Thermistoren

Zur Messung der Plattentemperaturen wurden NTC-Thermistoren verwendet. Diese wurden in den Platten platziert. Mit Hilfe eines Multimeters wurden die Widerstände der Thermistoren aufgenommen. Aus der Eichung (vgl. Abb. 7.10) konnte dann direkt die Temperatur bestimmt werden. 


\subsection{Fehlereinschätzung}

Da der Versuchsaufbau von [Hage, 2009] übernommen wurde, können für die Fehler die gleichen Annahmen für die Fehlerquellen gemacht werden.

Fingerdicke und chemische Rayleighzahlen bleiben daher bei einem Fehler von $10 \%$ und die Sherwoodzahl bei einem Fehler von 5\%, da hier die selben Messemethoden benutzt wurden. Für die Reynoldszahlen wurde wieder die Standardabweichung als Fehler genommen, die bei allen Messungen etwa $10 \%$ betrug.

Für die Temperaturmessung wurde eine Verbesserung durchgeführt, indem diese nun direkt in den Platten statt in den Thermostaten durchgeführt wird. Dadurch wird der Fehler der Temperaturmessung gegenüber der Messmethode von [Hage, 2009] gesenkt und mit $5 \%$ abgeschätzt. 


\section{Grenzen des Fingerregimes}

Teile dieses Kapitels wurden bereits in [Kellner and Tilgner, 2014] veröffentlicht.

\subsection{Vorüberlegungen}

Mit einer linearen Stabilitätsanalyse kann man die Grenzen der Fingerströmung betrachten. Eine gute Zusammenfassung dieser Rechnungen ist bei [Baines and Gill, 1969] zu finden. Dabei findet man im $R a_{c}-R a_{T}$-Raum ein Gebiet, in welchem Fingerströmung stattfinden kann. Dieses Gebiet wird durch die Gleichungen 3.1 und 3.2 eingegrenzt.

$$
\begin{aligned}
& R a_{T}=-L e^{-1} R a_{c} \longleftrightarrow \Lambda=-1 \\
& R a_{T}=-R a_{c}+27 \pi^{4} / 4 \quad=-R a_{c}+R a_{c, \text { crit }}
\end{aligned}
$$

Hier herrschen direkt wachsende Moden vor, d.h. der Imaginärteil der Störung ist 0 . Diese beziehen ihre Energie aus der potenziellen Energie der langsam diffundierenden Komponente. Für große chemische Rayleighzahlen $\left(R a_{c}>R a_{c, c r i t}\right)$ mit $R a_{T} \rightarrow-\infty$ und $L e \gg 1$ haben diese Moden eine Breite von

$$
d \sim\left(\frac{g \alpha_{T} \Delta T}{\kappa_{T} \nu d}\right)^{-1 / 4}
$$

Durch die Experimente von [Hage and Tilgner, 2010] und die numerischen Berechnungen von [Yang et al., 2015b] wird die Gültigkeit von Gleichung 3.1 in Frage gestellt. In ihren Untersuchungen fanden sie Finger in einem instabil geschichteten Medium, d.h. in einem Bereich mit $|\Lambda|<1$ bzw. $90^{\circ}<T u<135^{\circ}$.

Wieso können Finger im instabil geschichteten Medium entstehen und wann und wie verschwinden sie?

Schon die ersten Experimente haben gezeigt, dass Finger nicht beliebig lang werden können. Mögliche Ursachen werden im Kapitel 4.1 näher erläutert. Allerdings liefern sie 
keine Erklärungen, warum sie überhaupt entstehen bzw. warum sie zu Konvektionsrollen werden. [Hage, 2009] hat bezüglich letzterem einige Überlegungen gemacht:

- Die erste Möglichkeit besteht darin, dass der Übergang vom Fingerregime ins konvektive Regime kontinuierlich ist. Da die Finger nach Gleichung 3.4 immer breiter werden, wird irgendwann der Punkt, an dem $d=L$ gilt, erreicht. Dies geschieht bei einem Übergang von $\left|R a_{T}\right| \approx R a_{c}^{1 / 3}$.

- Nach Gleichung 3.6 wächst die Sherwoodzahl mit sinkendem Auftriebsverhältnis bis zu einem Punkt, an dem sich $S h_{\text {Finger }}>S h_{R B K}$ einstellt. Es ist von vornherein nicht ausgeschlossen, dass in Fingerströmung mehr Masse transportiert werden kann als in Rayleigh-Bénard-Konvektion. Trotzdem könnte dies einen möglichen Übergang darstellen. Dieser läge dann bei

$$
S h_{\text {Finger }} \approx S h_{R B K} \longleftrightarrow\left|R a_{T}\right| \approx 10^{-6} R a_{c}^{4 / 3}
$$

Auch [Schmitt, 2011] hat theoretische Überlegungen dazu durchgeführt und nachgewiesen, dass mit den vorhandenen Modellen für Fingerströmung ([Schmitt, 1979b] und [Schmitt, 1983]) Gleichung 3.1 auch ihre Gültigkeit verliert, solange die Prandtlzahl klein ist.

Für den Bereich $|\Lambda|<1$ im Fingerregime haben [Hage and Tilgner, 2010] Abhängigkeiten der Parameter $d / L, R e$ und $S h$ in Abhängigkeit der Rayleighzahlen gefunden, die im Wesentlichen von [Yang et al., 2015b] bestätigt wurden.

$$
\begin{aligned}
\frac{d}{L} & =0,95\left|R a_{T}\right|^{-1 / 3} R a_{c}^{1 / 9} \\
R e & =10^{-6}\left|R a_{T}\right|^{-1 / 2} R a_{c} \\
S h & =0,016\left|R a_{T}\right|^{-1 / 12} R a_{c}^{4 / 9}
\end{aligned}
$$




\subsection{Das Experiment}

Der Versuch ist im Wesentlichen eine Fortführung der Arbeit von [Hage, 2009].

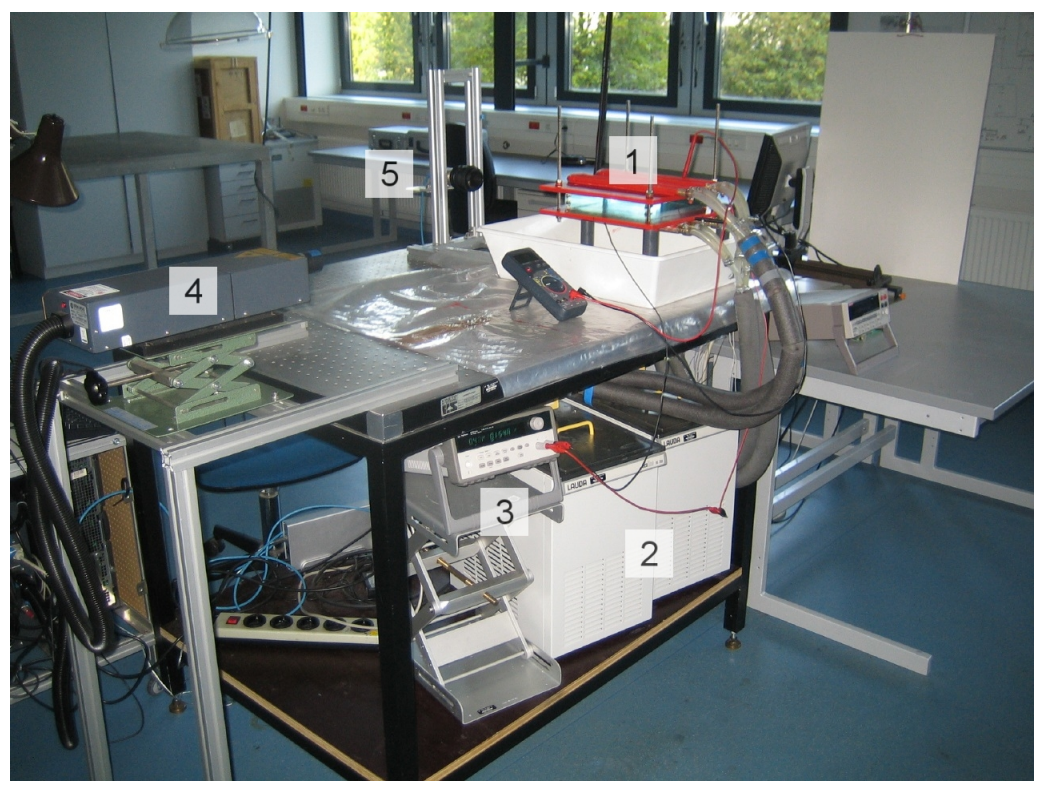

AвB. 3.1: Aufbau des Versuches mit der Zelle (1), den Thermostaten (2), der Spannungsquelle (3), dem Laser (4) und der Kamera (5). Abbildung aus [Hage, 2009].

\section{Aufbau}

Der Aufbau des gesamten Experimentes ist in Abbildung $3.1 \mathrm{zu}$ sehen. Die elektrochemische Zelle besteht aus einer kupfernen Bodenplatte und einem kupfernen Deckel mit jeweils den Maßen $30 \mathrm{~cm}$ mal $30 \mathrm{~cm}$. Die Seitenwände sind aus Plexiglas (in den Höhen $L=2,3,4,6,8$ und $20 \mathrm{~cm}$ ). Zur Abdichtung zwischen Seitenwand und Bodenplatte bzw. Deckel ist ein Gummiring eingelassen, der mit einer Silikonpaste bestrichen wurde. Die Fläche zwischen der Umrahmung beträgt $20 \mathrm{~cm}$ mal $20 \mathrm{~cm}$. Die Füße sind höhenverstellbar, sodass die Zelle immer horizontal ausgerichtet werden kann.

Die Kupferplatten sind gegen Korrosion und zur elektrischen Abschirmung von Außen mit Lack bestrichen. Die Temperatur der Platten kann über zwei Thermostate separat geregelt werden. An den Platten lässt sich eine Spannung über eine Spannungsquelle anlegen.

Als Fluid wird entgaste 1-molare Schwefelsäure, in der zuvor Kupfersulfatpentahydrat 
gelöst wurde, benutzt.

Für die optischen Messverfahren (siehe Kapitel 2.4) steht ein Laser bereit. Dieser ist mit der Kamera synchronisiert. Außerdem können Tracerpartikel oder Thermokristalle in die Zelle gefüllt werden. Die genauen Spezifikationen der technischen Bestandteile befinden sich im Apendix 7. Damit lassen sich chemische Rayleighzahlen im Bereich $\approx 3 \cdot 10^{8}$ bis $\approx 4 \cdot 10^{12}$, sowie thermische Rayleighzahlen im Bereich von $\approx 9 \cdot 10^{4}$ bis $\approx 5 \cdot 10^{12}$ vermessen.

\section{Bestimmung der Kennlinie}

Um die Kennlinie zu bestimmen, wird im Fingerregime bei konstantem Temperaturgradient die Spannung langsam erhöht und der Strom gemessen. Es ist wichtig nach jeder Erhöhung solange zu warten, bis sich das Konvektionsprofil den neuen Bedingungen angepasst hat. Dabei ist die Höhe des Temperaturgradienten egal, sodass wie in [Hage, 2009] ein Temperaturgradient von $\Delta T=-4 \mathrm{~K}$ genommen wurde. Allerdings hängt der Grenzstrom von der Kupferkonzentration $c_{0}$ und vom Plattenabstand $L$ ab. In Tabelle 3.1 können die entsprechenden Parameter für die Grenzströme, die für diese Arbeit benötigt werden, entnommen werden.

\begin{tabular}{|c|ccccccc|}
\hline$R a_{c} / 10^{10}$ & 0,100 & 0,243 & 1,73 & 2,92 & 13,9 & 18,5 & 216 \\
\hline$c_{0}[\mathrm{~mol} / \mathrm{l}]$ & 0,03 & 0,02 & 0,06 & 0,03 & 0,06 & 0,08 & 0,06 \\
$L[\mathrm{~m}]$ & 0,02 & 0,03 & 0,04 & 0,06 & 0,08 & 0,08 & 0,2 \\
\hline$U_{\text {eff }}[\mathrm{mV}]$ & 410 & 447 & 569 & 500 & 725 & 707 & 751 \\
\hline
\end{tabular}

TAB. 3.1: Spannungen, die zum Grenzstrom führen, für die sieben chemischen Rayleighzahlen 


\subsection{Ergebnisse}

\subsubsection{Bestimmung des Überganges}

Als erstes wurde ein Kriterium festgelegt, welches den Übergang vom Fingerregime zu Konvektion definiert. Um dies zu erreichen, muss man die Eigenschaften der Strömungen genauer betrachten:

- Die Kupferionenkonzentration ist im konvektiven Fall wie im Rayleigh-Bénard-Fall, d.h. im Zentrum ist sie annähernd homogen und an den Rändern liegt ein starker Gradient vor. Im Fingerregime hingegen hat jeder Finger eine definierte Konzentration an Kupferionen. Von der Anode kommend herrscht im Inneren des Fingers eine Konzentration von $c=2 \cdot c_{0}$ und von der Kathode kommend von $c=0$. Zwischen den Fingern gibt es einen Übergang, da die Ionen während der Durchquerung der Zelle auch diffundieren. Die Verteilung der Konzentrationen im Finger lässt sich schlecht bestimmen, da man wie bei [Hage, 2009] eine Probe nehmen muss, um diese dann auf ihre Absorption der Wellenlänge bei $800 \mathrm{~nm}$ zu untersuchen. Die Zelle direkt wie im Schadowgraphverfahren mit Licht der Wellenlänge $800 \mathrm{~nm} \mathrm{zu}$ durchleuchten liefert ein homogenes Bild, egal wie groß $\Delta c$ zwischen den Fingern ist. Das Streulicht auf dieser Wellenlänge ist zu schwach, um mit den zur Verfügung stehenden Mitteln detektiert zu werden. Daher ist die Konzentration als Kriterium ungeeignet.

- Die Geschwindigkeit liefert ein quantitativ bestimmbares Kriterium. Im konvektiven Fall, d.h. wenn sich Konvektionswalzen ausbilden, sind die x-Komponente und die y-Komponente im Mittel über die ganze Zelle gleich groß. Im Fingerregime dominiert die y-Komponente hingegen. Dies ist unter anderen durch das PIVVerfahren bedingt, da dieses nahe den Platten eine schlechte Auflösung hat, dort aber im Fingerregime die meiste Horizontalbewegung stattfindet.

Hält man nun den globalen Konzentrationsgradienten konstant, d.h. $R a_{c}=$ const. und variiert die Temperatur, so ergeben sich im Mittel über Zeit und Ort die Geschwindigkeiten, die in Abbildung $3.2 \mathrm{zu}$ sehen sind ${ }^{1}$. Hierbei wurde einmal die Reynoldszahl Re mit $v_{r m s}$ und einmal mit den Komponenten von $v_{r m s}$ gebildet.

$$
R e_{x}=\frac{L}{\nu}\left(\frac{1}{A} \int v_{x}^{2} d A\right)^{1 / 2} ; R e_{y}=\frac{L}{\nu}\left(\frac{1}{A} \int v_{y}^{2} d A\right)^{1 / 2} ; R e=\sqrt{R e_{x}^{2}+R e_{y}^{2}}
$$

\footnotetext{
${ }^{1}$ Abb. 7.1 bis 7.3 für die übrigen Messungen sind im Anhang
} 


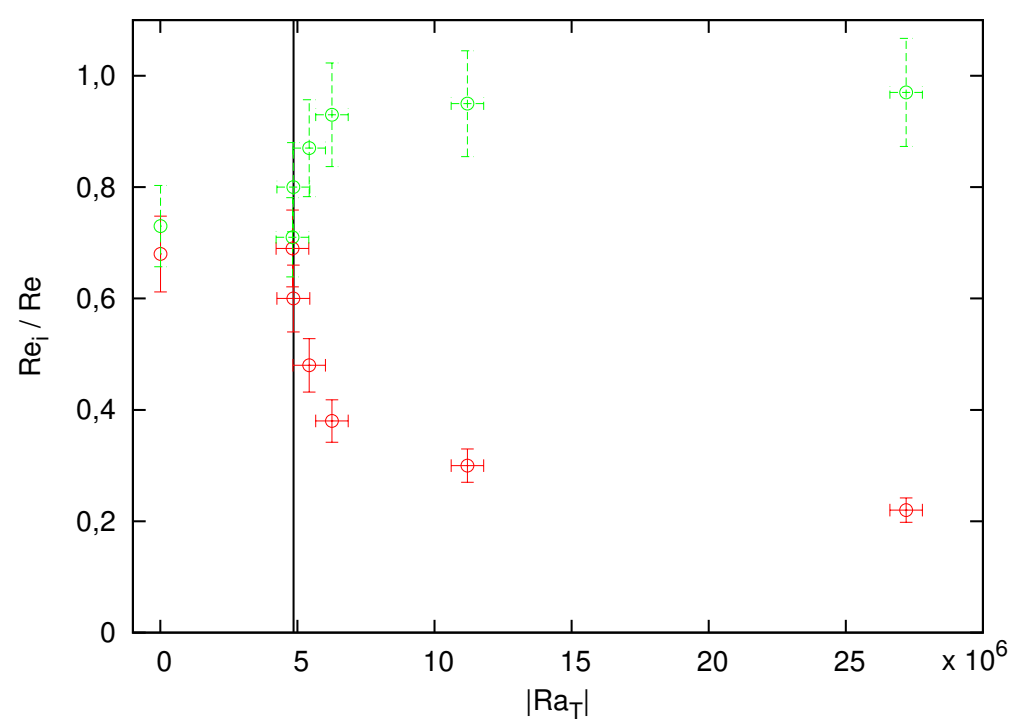

AвB. 3.2: $R e_{x} / R e$ (rot) und $R e_{y} / R e$ (grün) als Funktion von $\left|R a_{T}\right|$ bei konstanten $R a_{c}=2,92 \cdot 10^{10}$ aufgetragen. Die senkrechte Linie markiert den Übergang vom konvektiven zum Fingerregime.

Dabei erstreckt sich das Integral über die gesamte aufgenommene Fläche (typischerweise von Platte zu Platte in vertikaler und die Hälfte der Zelle in horizontaler Richtung). Im konvektiven Fall sollten $R e_{x}$ und $R e_{y}$ gleich groß sein, d.h. $R e_{x} / R e \approx R e_{y} / R e \approx 1 / \sqrt{2}$. Im Fingerregime sollte $R e_{y} \gg R e_{x}$ gelten. Man erkennt, dass es einen gut definierten Übergang gibt. Daher wurde die Geschwindigkeit als Übergangskriterium genommen. Vier exemplarische PIV-Aufnahmen für eine Messreihe sind in Abbildung 3.3 zu sehen. Die Geschwindigkeitsverteilung einer Momentaufnahme im Fingerregime und im konvektiven Regime sind in Abbildung 3.4 zu sehen.

Um den Übergang zu bestimmen, wird wie folgt vorgegangen. Zuerst wird für eine feste Konzentration $c_{0}$ wie in Kapitel 3.2 die Kennlinie bestimmt. Dann werden die Platten auf die entsprechenden Temperaturen eingestellt und gewartet, bis sich ein lineares Temperaturprofil in der Zelle ausgebildet hat. Tests haben ergeben, dass das Einstellen des linearen Temperaturgradienten nicht zwingend notwendig ist, da die Strukturen am Ende die gleichen waren egal, ob vorher ein linearer Temperaturgradient vorherrschte oder nicht. Nur die Zeit, bis der statistisch stationäre Fall eintritt, variiert mit den Anfangsbedingungen. Daher wurden für die Zellen mit $8 \mathrm{~cm}$ und $20 \mathrm{~cm}$ Anfangsbedingungen ohne linearen Temperaturgradienten gewählt. Nach Einstellen der Spannung beginnt das 

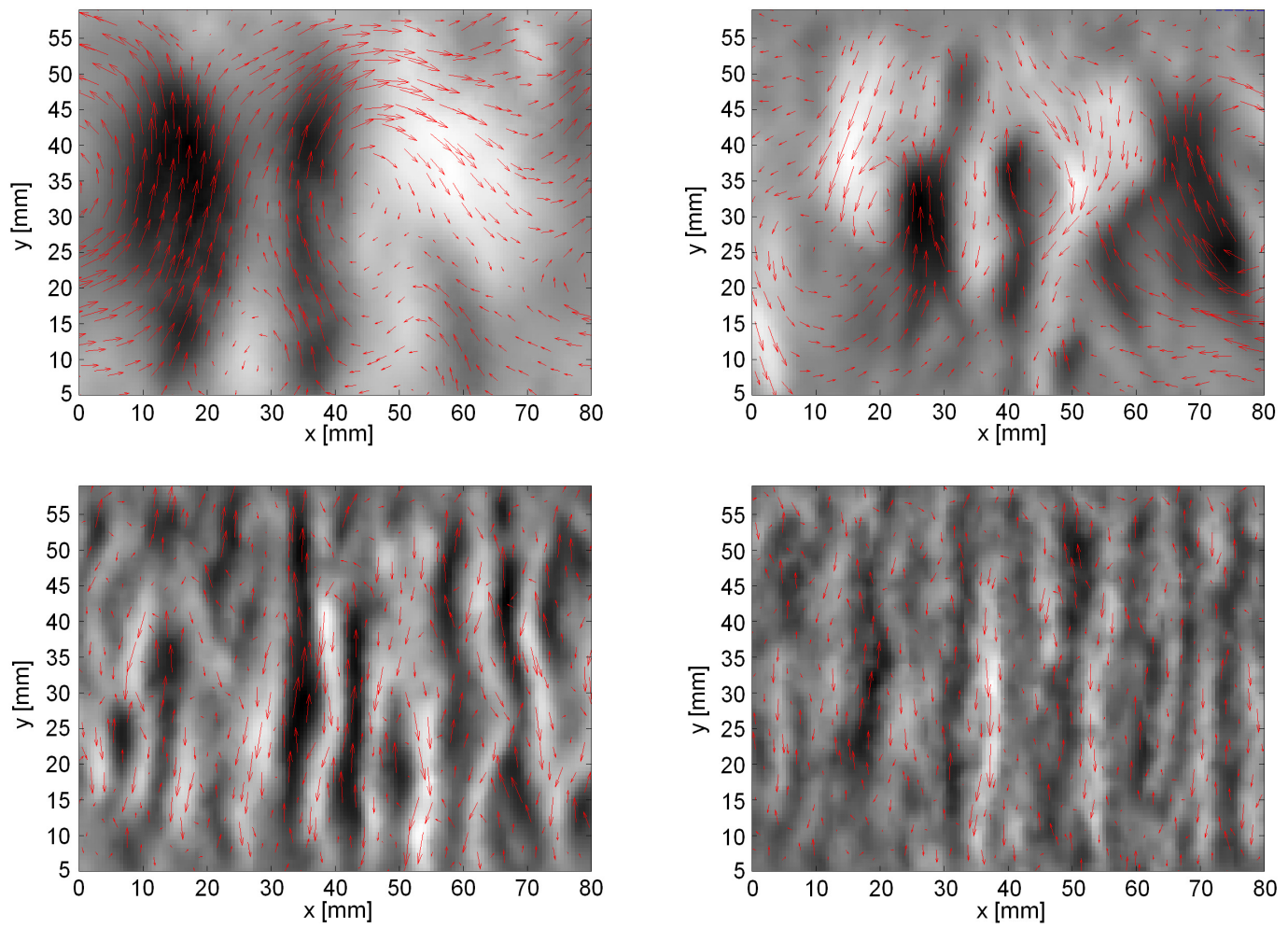

ABB. 3.3: Für $R a_{c}=2,92 \cdot 10^{10}$ ist das Geschwindigkeitsfeld (rote Pfeile) und die vertikale Geschwindigkeitskomponente (Schwarz nach oben, weiß nach unten) für die thermischen Rayleighzahlen $\left|R a_{T}\right|=0$ (links oben), $\left|R a_{T}\right|=4,85 \cdot 10^{6}$ (rechts oben), $\left|R a_{T}\right|=1,12 \cdot 10^{7}$ (unten links) und $\left|R a_{T}\right|=2,72 \cdot 10^{7}$ (unten rechts) in einer vertikalen Ebene dargestellt. Die Platten befinden sich bei $y=0 \mathrm{~mm}$ und $y=60 \mathrm{~mm}$. 

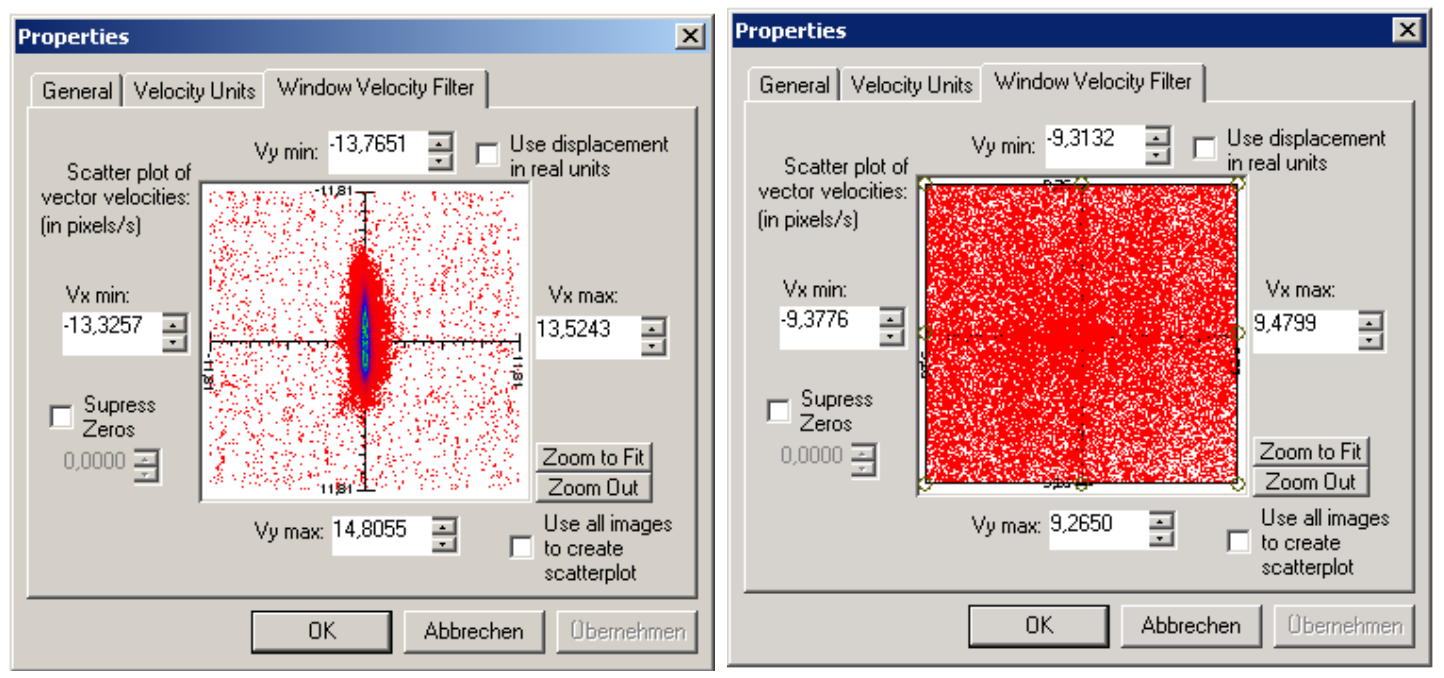

ABв. 3.4: Verteilung der Geschwindigkeitsvektoren im Fingerregime (links) und im konvektiven Regime (rechts). Man erkennt deutlich, dass das Verhältnis von $v_{y}$ zu $v_{x}$ rechts deutlich größer ist als links.

Die Abbildungen stammen aus dem Programm "VidPIV 4.1" und stellen das Menü für den globalen Filter dar. Per Hand wird der Bereich markiert, der kleine globale Ausreißer beinhaltet. Im Fingerregime erkennt man deutlich diese Ausreißer als einzelne rote Punkte außerhalb der zentralen Anhäufung. Diese Punkte werden dann in späteren Schritten interpoliert. Im konvektiven Regime macht dieser Filter wenig Sinn, sodass man hier nur den lokalen Filter zur Verfügung hat. 


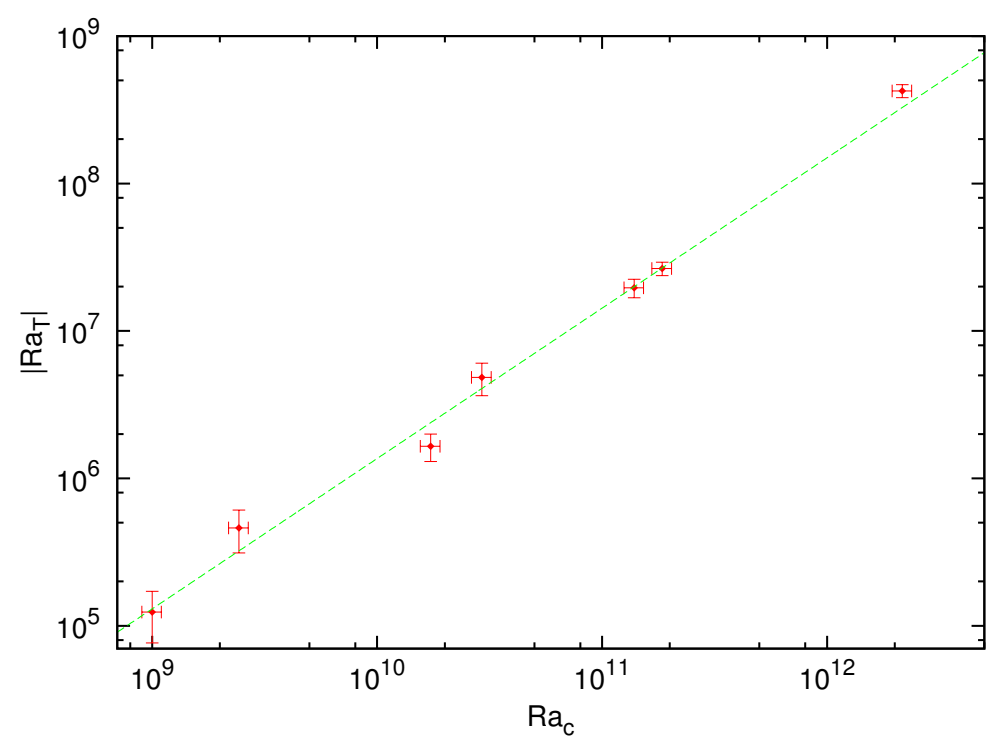

ABB. 3.5: Die Übergänge für die einzelnen chemischen Rayleighzahlen im $\left|R a_{T}\right|-\left.R a_{c}\right|^{-}$ Phasenraum. Die Symbole zeigen die gemessenen Übergänge und die gestrichelte Linie den besten Fit mit $\left|R a_{T}\right|=8,6 \cdot 10^{-5} R a_{c}^{1,02}$.

Profil sich einzustellen, das für die gegeben Parameter typisch ist. Mittels PIV wird die Geschwindigkeit im Anschluss vermessen. Dies wird für mehrere $\left|R a_{T}\right|$ durchgeführt, wobei $\left|R a_{T}\right|=0$ und $\left|R a_{T}\right| \gg\left|R a_{T}\right|_{\text {crit }}$ auch mit vermessen werden. Für jedes $\left|R a_{T}\right|$ wird der Versuch neu aufgebaut. Im Schachtelverfahren werden dann Messungen durchgeführt, die sich immer mehr dem Übergang nähren. Als Abbruchbedingung wird die Differenz zweier Messpunkte gewählt. Ist diese kleiner als die Messgenauigkeit, wird der Mittelwert der beiden Messpunkte als $\left|R a_{T}\right|_{\text {crit }}$ definiert.

Dabei besteht allerdings immer noch die Gefahr, dass Hysteresen auftauchen können. Um dies auszuschließen, wurde zuerst ein $\left|R a_{T}\right|$ im Fingerregime eingestellt. Danach wurde die Temperaturdifferenz, also $\left|R a_{T}\right|$ verringert. Dabei wurden in regelmäßigen Abständen PIV-Aufnahmen gemacht. $\left|R a_{T}\right|$ wurde soweit verringert, dass man deutlich im konvektiven Regime war. Diese Prozedur wurde auch für die andere Richtung durchgeführt. Dabei stellten sich immer die gleichen Finger bei den gleichen Parametern ein, sodass eine Hysterese ausgeschlossen werden kann. Damit ist der Übergang vom Fingerregime zur Konvektion eindeutig als überkritische Bifurkation bestimmt.

Für sieben chemische Rayleighzahlen wurden die kritischen thermischen Rayleighzahlen bestimmt. Diese sind in Abbildung 3.5 dargestellt. Man erkennt deutlich, dass der Über- 
gang bei $R a_{T} / R a_{c} \approx$ const. bzw. bei $\Lambda=-1 / 30$ erfolgt. Der Fit ergibt ein Potenzgesetz mit $^{2}$ :

$$
\left|R a_{T}\right|=8,6 \cdot 10^{-5} R a_{c}^{1,02} .
$$

Somit erfolgt der Übergang von Konvektion ins Fingerregime bei einem konstanten $\Lambda$. Jedoch nicht bei $|\Lambda|=1$, wie z.B. von [Turner, 1974] postuliert, sondern bei $|\Lambda|=1 / 30$ in einem Bereich von ca. 3 Größenordungen für $R a_{c}$.

\subsubsection{Eigenschaften des Systems am Übergang}

Parallel zur Bestimmung des Überganges können einige physikalische Größen analysiert werden.

\section{Die Sherwoodzahl}

Wie [Hage and Tilgner, 2010] schon gezeigt haben, steigt die Sherwoodzahl mit abnehmenden Auftriebsverhältnis nach Gleichung 3.6. Dieses Potenzgesetz verliert seine Gültigkeit im konvektiven Regime wie von [Hage and Tilgner, 2010] schon vermutet. Allerdings schafft es die Fingerströmung höhere Sherwoodzahlen zu erzeugen als durch reine Konvektion. Die Sherwoodzahl in Abhängigkeit vom Auftriebsverhältnis ist in Abbildung 3.6 zu sehen ${ }^{3}$. Eigentlich erwartet man, dass die Konvektion mit ihren höheren Geschwindigkeiten mehr Ionen von Platte zu Platte transportieren kann als es den Fingern möglich ist. Jedoch beschränkt sich der Transport der Ionen in den Bereichen mit vertikaler Geschwindigkeit und diese ist bei den Konvektionswalzen nur auf einen kleinen Bereich am Rand beschränkt. Finger existieren jedoch in der ganzen Zelle, sodass es vertikale Bewegung in einem Bereich gibt, der $50 \%$ der Grundfläche ausmacht. Finger sind zwar immer noch langsamer als die Konvektionswalzen, aber dies können sie besonders nahe des Überganges durch ihre hohe Anzahl ausgleichen. Des Weiteren gilt die Gleichung 3.6 nahe des Überganges nicht mehr. Vor allem für kleine chemische Rayleighzahlen gibt es größere Abweichungen (siehe Abb. 3.7 (links). Der beste Fit durch die Daten $(0,03<|\Lambda|<0,37)$ ergibt $S h=0,11 R a_{c}^{0,37} R a_{T}^{-0,08}$.

\footnotetext{
${ }^{2}$ Setzt man den Exponenten auf 1 fest, so ergibt sich ein Vorfaktor von $1.4 \cdot 10^{-4}$ für den Fit.

${ }^{3}$ Abb.7.4 bis 7.6 für die übrigen Messungen befinden sich im Anhang
} 


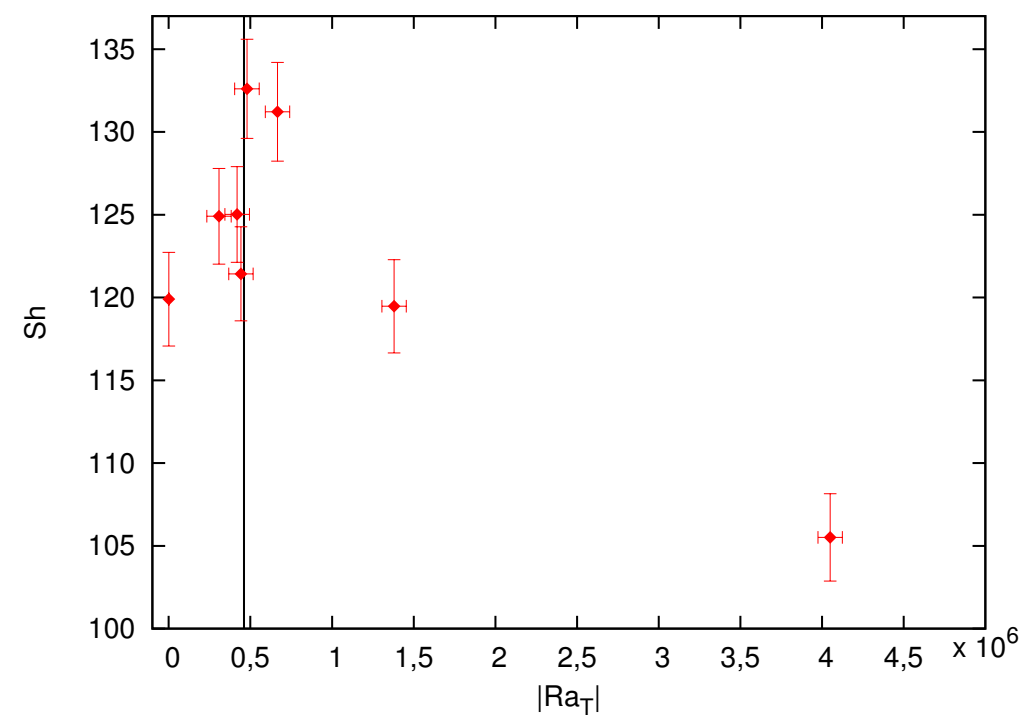

ABB. 3.6: $S h$ als Funktion von $\left|R a_{T}\right|$ bzw. $|\Lambda|$ für $R a_{c}=2,43 \cdot 10^{9}$. Die Maximale Sherwoodzahl wird in der Nähe des Überganges vom Fingerregime zum konvektiven Regime erreicht. Dieser ist durch die senkrechte Linie markiert
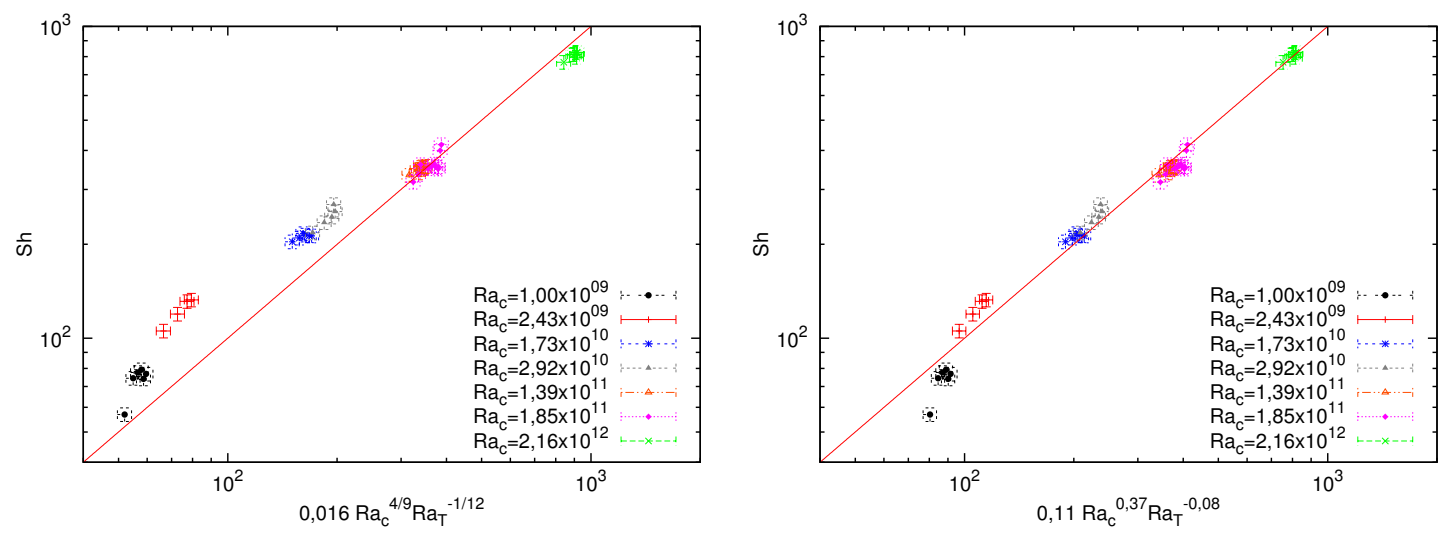

ABB. 3.7: LINKS: Aufgetragen ist Gleichung 3.6 (rote Linie) für alle Datenpunkte im Fingerregime. Man erkennt, dass es Abweichungen vor allem für kleine chemische Rayleighzahlen gibt.

RECHTS: Aufgetragen ist der beste Fit der Daten am Übergang mit $S h=$ $0,11 R a_{c}^{0,37} R a_{T}^{-0,08}$. 
Diese Exponenten genügen allerdings nicht der Bedingung 5.15 aus [Hage, 2009]. Daher ist nicht gesichert, ob die Annahmen, die zu Gleichung 3.6 führten, noch Gültigkeit haben. Vor allem wurde argumentiert, dass Fluidpartikel nur den vertikalen Temperaturgradienten sehen, da die Temperatur schnell diffundiert. Wie sich das Temperaturfeld am Übergang verhält wird im Kapitel 3.3.3 näher betrachtet. Es zeigt sich, dass am Übergang zwischen den Fingern auch ein horizontaler Gradient entsteht. Der Temperaturunterschied zwischen den Fingern ist zwar kleiner als zwischen den Platten, aber wahrscheinlich nicht mehr vernachlässigbar.

[Yang et al., 2015a] haben in numerischen Simulationen auch eine höhere Sherwoodzahl im Fingerregime als im konvektiven Regime gefunden, jedoch sind ihre Maxima im Bereich $|\Lambda| \approx 1$. Für diese Diskrepanz konnte bis jetzt keine Ursache gefunden werden.

Da für jede der sieben chemischen Rayleighzahlen auch bei $R a_{T}=0$ gemessen wurde, konnten die Sherwoodzahlen für reine Rayleigh-Bénard-Konvektion bestimmt werden. Diese sind in Abbildung 3.8 zu sehen. Der beste Fit an die Daten ergibt:

$$
S h=0,19 \cdot R a_{c}^{0,29} .
$$

Obwohl der Exponent sehr nahe an den Ergebnissen von [Xia et al., 2002] liegt (innerhalb des Fehlerintervalls), die mit Temperatur im Wasser und organischen Lösungen durchgeführt wurden, sind die Werte kleiner als in dieser Arbeit. Man muss hier aber berücksichtigen, dass die $\operatorname{Pr}$ um einen Faktor 2 größer ist als die höchste verwendete Prandtlzahl und, dass das Aspektverhältnis $\Gamma$ bei [Xia et al., 2002] konstant 1 ist. Solche Abweichungen sind bei den hohen Rayleighzahlen in verschiedenen Experimenten nicht unüblich (vgl. u.a. [Ahlers et al., 2009]). Die Großmann/Lohse-Therorie [Grossmann and Lohse, 2001] sagt für den Bereich $R a_{c}>3 \cdot 10^{9}$ einen Exponenten von $1 / 3$ in Übereinstimmung der Ergebnisse von [Hage, 2009] vorher. Allerdings wurden diese Experimente für ein konstantes Aspektverhältnis von $\Gamma=1$ gemacht. In dieser Arbeit variiert das $\Gamma$ von 1 bis 10. Sodass auch hier dadurch Abweichungen entstehen können. Eine genauere Untersuchung und weitere Messpunkte wären nötig, um genaue Aussagen zu tätigen. Da dieser Abschnitt aber nicht im Fokus der Arbeit war, sondern nur ein Nebenprodukt, muss es hierbei belassen werden. 


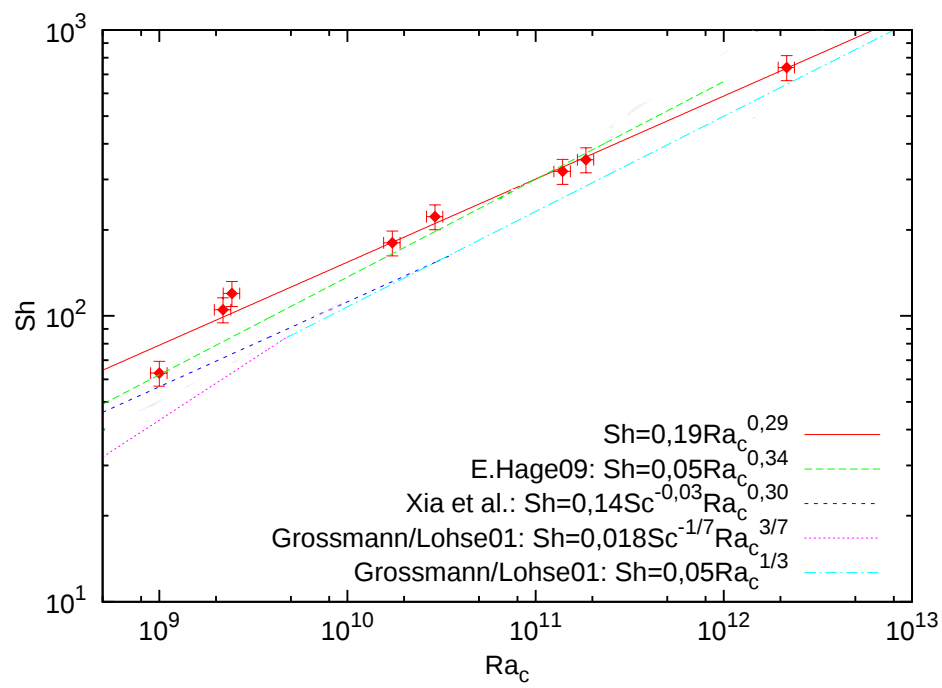

ABB. 3.8: $S h$ wird gegen $R a_{c}$ für $R a_{T}=0$ (rote Punkte) mit bestem Fit (rote Linie) aufgetragen. Man erkennt Übereinstimmung im Exponenten mit den Ergebnissen von [Xia et al., 2002] (blau) aber Abweichungen im Vorfaktor. Auch die Großmann/Lohse Theorie (magenta für $R a_{c}<3 \cdot 10^{9}$ und hellblau für $\left.R a_{c}>3 \cdot 10^{9}\right)$ hat Abweichungen im Exponenten und Vorfaktor. Zum Vergleich sind noch die Ergebnisse von [Hage, 2009] (grün) dargestellt, die mit dem gleichen Experiment, aber konstanten $\Gamma$ gemessen hat.

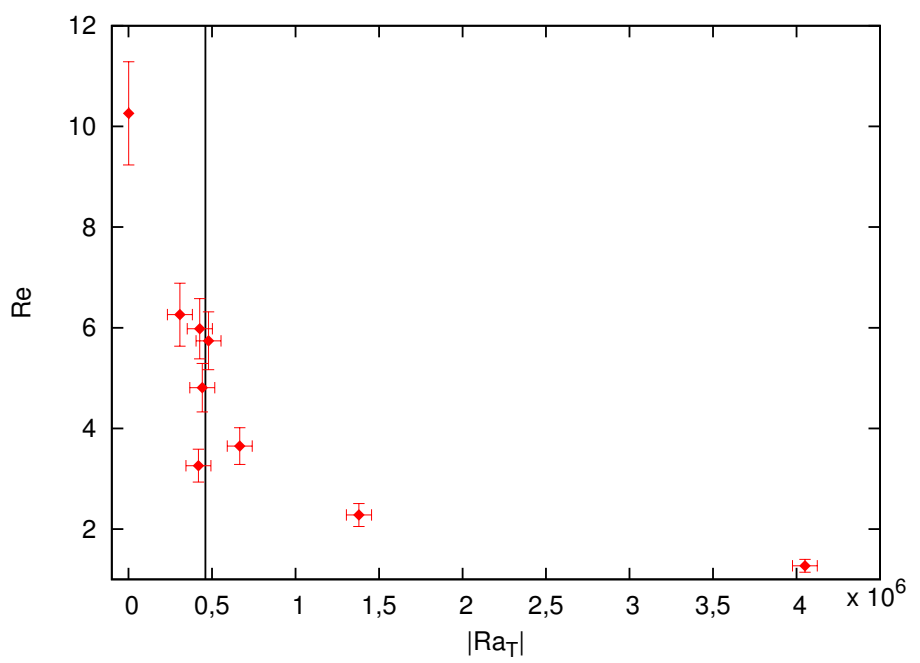

AвB. 3.9: $R e$ gegen $\left|R a_{T}\right|$ nahe des Überganges (senkrechter schwarzer Strich) für $R a_{c}=$ $2,43 \cdot 10^{9}$. Die Geschwindigkeit der Fingerströmung steigt mit abnehmender $\left|R a_{T}\right|$ und hat das Maximum im Konvektionsregime bei $\left|R a_{T}\right|=0$. 

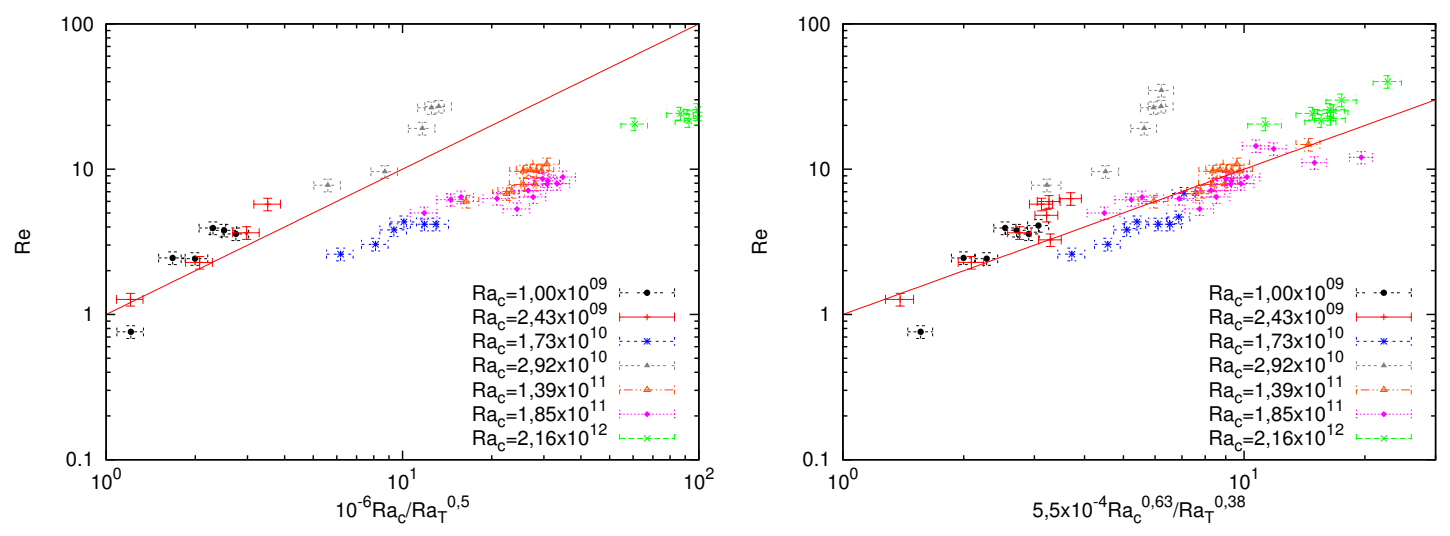

AвB. 3.10: LINKS: Aufgetragen ist Gleichung 3.5 (rote Linie) für alle Datenpunkte im Fingerregime. Man erkennt, dass der Verlauf qualitativ noch stimmt, der Vorfaktor aber für jede chemische Rayleighzahl variiert werden müsste.

RECHTS: Aufgetragen ist der beste Fit der Daten am Übergang mit $R e=$ $5,5 \cdot 10^{-4} R a_{c}^{0,63} R a_{T}^{-0,38}$. Bis auf die Ausnahme $R a_{c}=2,92 \cdot 10^{10}$ streuen die Daten nur gering um den Fit.

\section{Die Reynoldszahlen}

Die Reynoldszahlen steigen, wie in Abbildung $3.9 \mathrm{zu} \mathrm{sehen}^{4}$, mit sinkendem $\left|R a_{T}\right|$. Dies gilt sowohl für das Fingerregime als auch für das konvektive Regime. Die maximale Reynoldszahl wird bei $\left|R a_{T}\right|=0$ erreicht, d.h. sie ist maximal, wenn der stabilisierende Einfluss der Temperatur minimal ist (verschwindet). Die Reynoldszahlen haben zwar noch den qualitativen Verlauf nach Gleichung 3.5, allerdings müsste der Vorfaktor teilweise um bis zu einen Faktor 4 angepasst werden, damit die Kurven den Verlauf der Punkte gut wiedergeben. Dies ist in Abbildung 3.10 (links) dargestellt. Der beste Fit durch die Datenpunkte $(0,03<|\Lambda|<0,37)$ ergibt $R e=5,5 \cdot 10^{-4} R a_{c}^{0,63} R a_{T}^{-0,38}$. Wie schon bei der Sherwoodzahl genügen diese Exponenten nicht der Bedingung 5.43 aus [Hage, 2009]. Hier gelten die gleichen Überlegungen, wie bei der Sherwoodzahl bezüglich der Annahmen, die zur Bedingung 5.43 führten. Bis auf die Messung für $R a_{c}=2,92 \cdot 10^{10}$ ist die Streuung der Messdaten um den Fit in der Größenordnung wie bei [Hage, 2009].

\footnotetext{
${ }^{4}$ Abb. 7.7 bis 7.9 für die übrigen Messungen befinden sich im Anhang
} 


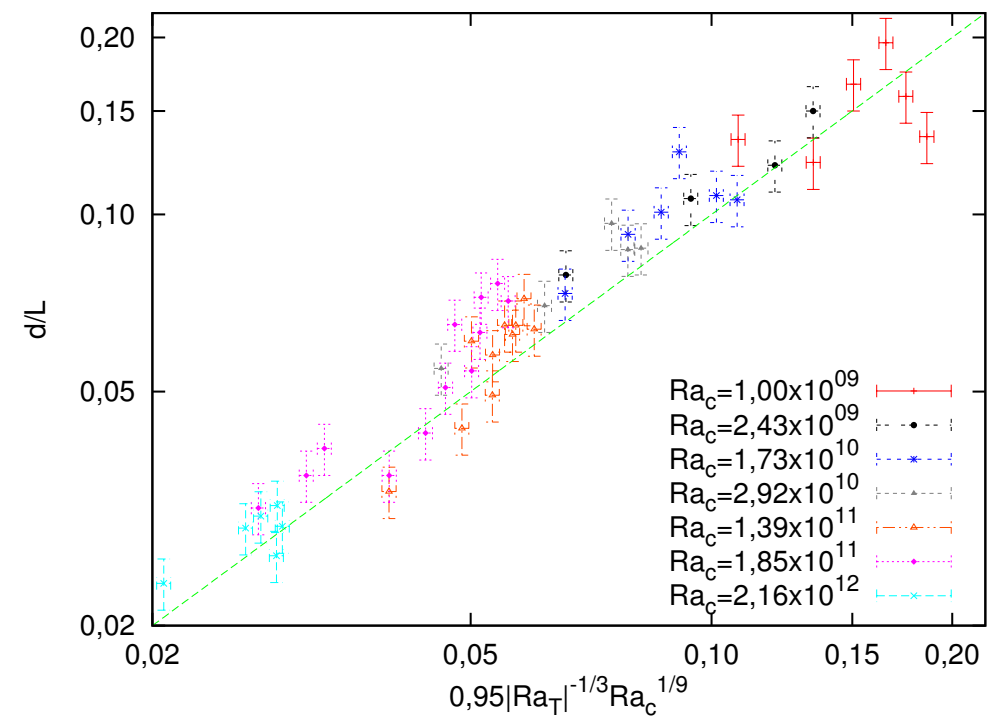

AвB. 3.11: Die normierte Fingerdicke aufgetragen gegen Gleichung 3.4. Man erkennt, dass alle Punkte nahe der grünen Linie mit $d / L=0,95\left|R a_{T}\right|^{-1 / 3} R a_{c}^{1 / 9}$ liegen, aber etwas parallel dazu verschoben sind. Eine geringe Angleichung (5\%) des Vorfaktors innerhalb der Fehlertoleranz des Fits würde dies beheben.

\section{Die Fingerdicken}

Die Fingerdicken steigen mit abnehmendem Auftriebsverhältnis wie erwartet an. In Abbildung 3.11 sieht man, dass sich die Finger bis zum Übergang nach Gleichung 3.4 verhalten. Die Datenpunkte sind aber tendenziell etwas zu groß. Eine Korrektur des Vorfaktors von Gleichung 3.4 um 5\% nach oben würde dies wieder ausgleichen. Dies liegt noch innerhalb der Messgenauigkeit und des Fitfehlers. Daher kann die Gültigkeit von Gleichung 3.4 bis zum Übergang vom Fingerregime zum konvektiven Regime als bestätigt gelten. 


\subsubsection{Mechanismen für den Übergang}

Da Finger auch für Auftriebsverhältnisse $|\Lambda|<1$ existieren, d.h. selbst wenn die gesamte Schichtung des Fluides instabil ist, stellt sich die Frage, welcher Mechanismus dafür sorgt, dass die Finger zu Konvektionswalzen werden. Der Zerfall von Fingern wurde schon häufig besonders im Bezug auf die Schichtbildung untersucht (vgl. u.a. [Stern, 1969, Stern and Turner, 1969, Radko, 2003, Stellmach et al., 2011]. Dabei wurde jedoch hauptsächlich der Fingerzerfall bei konstantem Auftriebsverhältnis untersucht. Dieses war stets größer eins und somit das Fluid stabil geschichtet. Diese Thematik wird noch im Kapitel 4 behandelt.

[Müller, 2013] hat im Bereich $R a_{c}=10^{4}-10^{6}$ für $L e=10$ und $L e=100$ den Übergang vom Fingerregime zur Konvektion numerisch untersucht. Für $L e=10$ wandern die Übergänge von $|\Lambda|=1$ für $R a_{c}=10^{4} \mathrm{zu}$ immer kleineren Auftriebsverhältnissen bei steigender $R a_{c}$ und nähern sich $|\Lambda|=1 / 6$ für $R a_{c}>2 \cdot 10^{5}$. Die Daten deuten darauf hin, dass mit steigender $R a_{c}$ diese Grenze nicht unterschritten wird. Für $L e=100$ wandern die Übergänge auch, startend von $|\Lambda|=1$ bei $R a_{c}=10^{4}$, zu $|\Lambda|=1 / 30$ bei $R a_{c}=10^{6}$. Er konnte jedoch nicht zeigen, ob die Grenze $|\Lambda|=1 / 30$ bis $R a_{c}=10^{9}$ bleibt oder sich ändert, bzw. einen Mechanismus für die Übergänge finden.

[Schmitt, 2011] hat basierend auf den Ergebnissen von [Schmitt, 1979b] und [Schmitt, 1983] Instabilitäten in einem unendlich ausgedehnten Medium untersucht. Die am schnellsten wachsende Instabilität der linearen Hintergrundfelder (Temperatur und Salz) ist vom Fingertyp (elevator mode), wenn $|\Lambda|>0,9$ für $\operatorname{Pr}=8,68$ und $L e=227$ ist. Danach sollten in diesem Experiment nur Finger auftauchen, wenn das Auftriebsverhältnis größer 0,9 ist. Somit lässt das Modell zwar Finger für instabile Auftriebsverhältnisse zu, jedoch nicht bis zu $|\Lambda|=1 / 30$.

[Stern, 1969] und [Holyer, 1981] haben für stabile Auftriebsverhältnisse $(|\Lambda|>1)$ gezeigt, dass Finger instabil bezüglich langer interner Wellen werden, wenn ihre vertikale Ausdehnung zu groß wird. Aus den Gleichungen (2.1),(2.2),(2.3) und (4.4) von [Stern, 1969] und aus Gleichung (1.1) von [Holyer, 1981] kann die Relation 3.9 (Sternkriterium) hergeleitet werden.

$$
R e_{f}=R e_{y} \frac{d}{L} \geq \sqrt{\frac{2}{3} \frac{R a_{T}}{\operatorname{Pr}}\left(\frac{d}{L}\right)^{4}}
$$




\begin{tabular}{|c|c|c|c|c|c|c|c|c|}
\hline$R a_{c}$ & $\left|R a_{T}\right|$ & $\Lambda$ & $R e$ & $R e_{f}$ & $R a_{c, \lambda}$ & $\sqrt{\kappa L /\left(v d^{2}\right)}$ & $\sqrt{\frac{2}{3} \frac{R a_{T}}{P r}\left(\frac{d}{L}\right)^{4}}$ & $R a_{\lambda}$ \\
\hline \hline $1,00 \cdot 10^{09}$ & $1,33 \cdot 10^{5}$ & 0,030 & 03,6 & 0,59 & 138 & 1,45 & 1,87 & 13,8 \\
& $1,15 \cdot 10^{5}$ & 0,026 & 04,1 & & 140 & & & 14,2 \\
\hline $2,43 \cdot 10^{09}$ & $4,79 \cdot 10^{5}$ & 0,044 & 05,7 & 0,73 & 065 & 1,03 & 4,34 & 03,4 \\
& $4,42 \cdot 10^{5}$ & 0,041 & 04,8 & & 085 & & & 04,9 \\
\hline $1,73 \cdot 10^{10}$ & $1,78 \cdot 10^{6}$ & 0,024 & 04,2 & 0,39 & 112 & 1,66 & 4,52 & 03,8 \\
& $1,56 \cdot 10^{6}$ & 0,021 & 04,7 & & 112 & & & 03,8 \\
\hline $2,92 \cdot 10^{10}$ & $4,85 \cdot 10^{6}$ & 0,037 & 18,9 & 1,32 & 110 & 1,01 & 4,77 & 03,0 \\
& $4,82 \cdot 10^{6}$ & 0,037 & 34,9 & & 119 & & & 03,4 \\
\hline $1,39 \cdot 10^{11}$ & $2,05 \cdot 10^{7}$ & 0,034 & 10,8 & 0,63 & 192 & 1,69 & 5,15 & 03,8 \\
& $1,96 \cdot 10^{7}$ & 0,032 & 09,7 & & 191 & & & 03,8 \\
\hline $1,85 \cdot 10^{11}$ & $2,83 \cdot 10^{7}$ & 0,035 & 08,8 & 0,60 & 159 & 1,66 & 7,58 & 02,7 \\
& $2,47 \cdot 10^{7}$ & 0,031 & 14,5 & & 181 & & & 03,3 \\
\hline $2,16 \cdot 10^{12}$ & $4,51 \cdot 10^{8}$ & 0,048 & 25,2 & 0,62 & 240 & 2,28 & 5,18 & 02,0 \\
& $3,98 \cdot 10^{8}$ & 0,042 & 29,9 & & 245 & & & 02,1 \\
\hline
\end{tabular}

TAB. 3.2: Parameter der Messungen direkt vor und nach dem Übergang vom Fingerregime zum konvektiven Regime. Aufgelistet sind: chemische Rayleighzahl $R a_{c}$, thermische Rayleighzahl $R a_{T}$, Auftriebsverhältnis $\Lambda$, Reynoldszahl Re, Fingerreynoldszahl $R e_{f}=R e_{y} d / L$, die chemische Rayleighzahl der chemischen Grenzschicht $R a_{c, \lambda}=R a_{c} /\left(16 S h^{3}\right)$, das Verhältnis von thermischer Diffusionslänge zur Fingerdicke $\sqrt{\kappa L /\left(v d^{2}\right)}$, das Sternkriterium und die Grenzschichtrayleighzahl $R a_{\lambda}$. Für Werte im konvektiven Regime konnten keine Fingerdicken bestimmt werden. Daher wurden alle Werte, die diese zur Berechnung brauchen, nicht aufgelistet. 
Sobald diese Bedingung erfüllt ist, haben die Wellen steigende Anwachsraten und die Finger werden zerstört. In Tabelle 3.2 ist diese Bedingung für die Messpunkte am Übergang eingetragen. Man erkennt, dass dieses Kriterium nie erfüllt ist und daher nicht die Ursache für den Übergang sein kann. Eine Erweiterung des Modells durch [Holyer, 1984] auf kurze Wellen zeigt, dass die Anwachsraten dadurch steigen. Allerdings gibt es kein analytisches Kriterium, welches hier getestet werden kann. Zudem bezieht sich die gesamte Arbeit auf den Bereich $|\Lambda|>1$, dem klassischen Fingerregime. Dadurch lässt sich nicht feststellen, ob die Ergebnisse auch für diese Arbeit relevant sind.

Die Finger sind am Übergang auch hydrodynamisch stabil. Dies lässt sich aus Tabelle 3.2 ablesen. Die Fingerreynoldszahl $R e_{f}$ ist bis auf eine Ausnahme kleiner 1. Daher ist es unwahrscheinlich, dass eine hydrodynamische Instabilität innerhalb der einzelnen Finger für den Übergang zur Konvektion führt. Die chemische Grenzrayleighzahl (mit Dicke $L /(2 S h)$ ) lässt sich über $R a_{c} /(16 S h)^{3}$ berechnen und ist auch in Tabelle $3.2 \mathrm{zu}$ finden. Auch hier sind die Werte zu gering, um Instabilitäten auszulösen. [Radko and Stern, 2000] haben eine Grenzschichtrayleighzahl $R a_{\lambda}$ mit

$$
R a_{\lambda}=\frac{g \lambda^{4}}{\nu}\left(\frac{\Delta c}{\kappa_{c} L}-\frac{\Delta T}{\kappa_{T} L}\right)
$$

definiert. Dabei ist $\lambda$ die Grenzschichtdicke. Erreicht diese Rayleighzahl ca. 1000, so wird die Grenzschicht instabil und kann das Bilden von Fingern unterdrücken. $R a_{\lambda}$ wurde mit der chemischen Grenzschichtdicke $(L /(2 S h))$ gebildet. Die Grenzschichtrayleighzahlen am Übergang können aus Tabelle 3.2 entnommen werden. Man erkennt, dass man noch weit vom kritischen Punkt entfernt ist. Die chemische Grenzschichtdicke müsste um einen Faktor 5 größer sein und damit die Sherwoodzahl um diesen Faktor kleiner, um kritische Grenzschichtrayleighzahlen zu erreichen. Man kann die Finger noch als KolmogorovStrömung mit $\vec{v}=v_{y 0} \sin (\pi x / d) \vec{y}$ nähern. Dabei ist $\vec{y}$ der Einheitsvektor in $y$-Richtung. Nach [Green, 1974] ist diese Strömung stabil, wenn $v_{y 0} d / \nu<\pi \sqrt{2}$ bzw. $R e_{f}<\pi$ ist (da $R e_{f}$ mit dem "root mean square" statt dem Maximum der Geschwindigkeit definiert ist). Dies ist immer der Fall. Daher gibt es keinen deutlich zu erkennenden Weg, die Finger mechanisch zu destabilisieren.

Finger können sich nur formen, wenn ein stabiles Temperaturfeld vorliegt. Da aber die Geschwindigkeit der Fingerströmung mit abnehmendem Auftriebsverhältnis zunimmt, könnte das Temperaturfeld dadurch immer mehr zerstört werden, bis die Finger zerfallen. Daher wurde das Temperaturfeld für $R a_{c}=2,92 \cdot 10^{10}$ mit Thermokristallen 


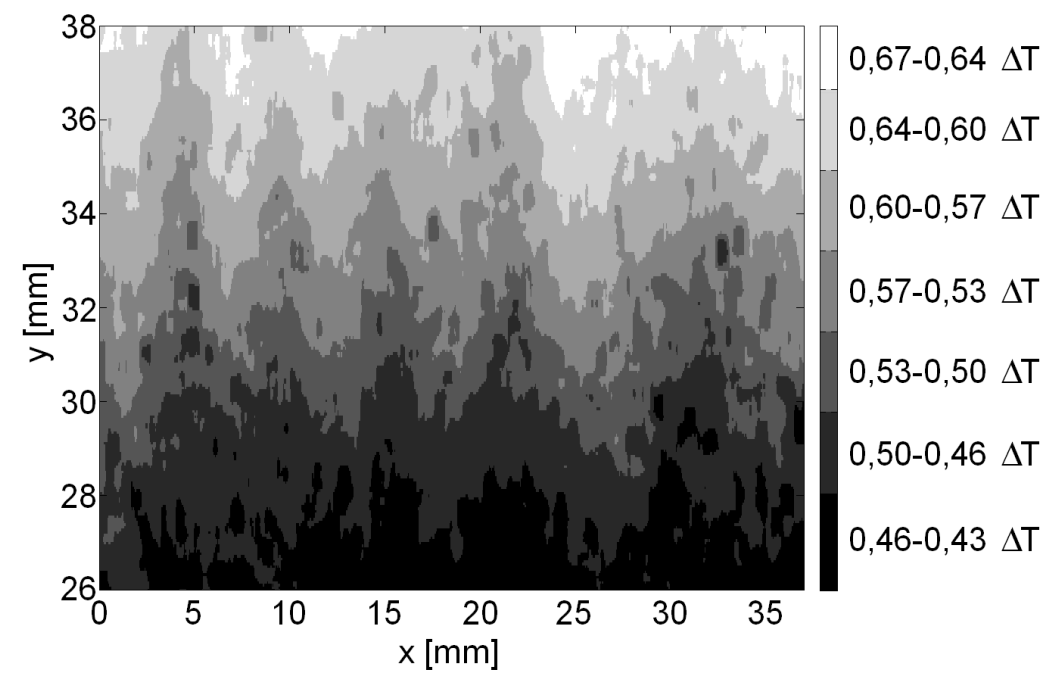

AвB. 3.12: Gray scale Plot des Temperaturfeldes in einer vertikalen Ebene für $R a_{c}=$ $2,92 \cdot 10^{10}$ und $\left|R a_{T}\right|=1,86 \cdot 10^{7}$. Der Übersicht wegen wurden die Temperaturen in Bins aufgeteilt. Die Platten befinden sich bei $y=0 \mathrm{~mm}$ und $y=60 \mathrm{~mm}$.

sichtbar gemacht. In Abbildung 3.12 ist dies beispielhaft dargestellt. Man erwartet im Fingerregime, dass die Isothermen durch die Konvektion sinusformig gestört werden. Da die Plattentemperaturen gleich bleiben, steigt der Temperaturgradient bei aufsteigenden Fingern nahe der oberen Platte an und wird nahe der unteren Platte kleiner (bzw. umgekehrt für fallende Finger). Deshalb wird durch die Fingerströmung die stabile Schichtung global nicht gestört, solange die Amplitude der Störung der Isothermen kleiner ist als die Zellhöhe. Im konvektiven Regime stellt sich außerhalb der Grenzschichten eine mittlere Temperatur ein. Dies kann auch derart interpretiert werden, dass die Störung der Isotherme mit der Temperatur $0,5 \Delta T$ (definiert als $T-T_{\text {unten }}$ ) größer ist als die Zelle. Der Abstand zwischen der maximalen und minimalen Höhe der Isotherme wird als $2 \mathrm{~A}$ definiert und ist für verschiedene thermische Rayleighzahlen normiert mit der Zellhöhe in Abbildung 3.13 dargestellt ${ }^{5}$. Man erkennt, dass die Störung der Isothermen mit abnehmendem Auftriebsverhältnis zunimmt. Allerdings reicht die Störung am Übergang nicht aus, um den Temperaturgradienten außerhalb der Grenzschichten zu zerstören. An einigen Stellen ist er sogar größer als der globale Gradient.

Finger entstehen, wenn die Wärmediffusion schnell genug ist, um Wärme zwischen be-

\footnotetext{
$\overline{{ }^{5} \text { Dargestellt ist aus technischen Gründen }}$ die Linie $T=0,51$.
} 


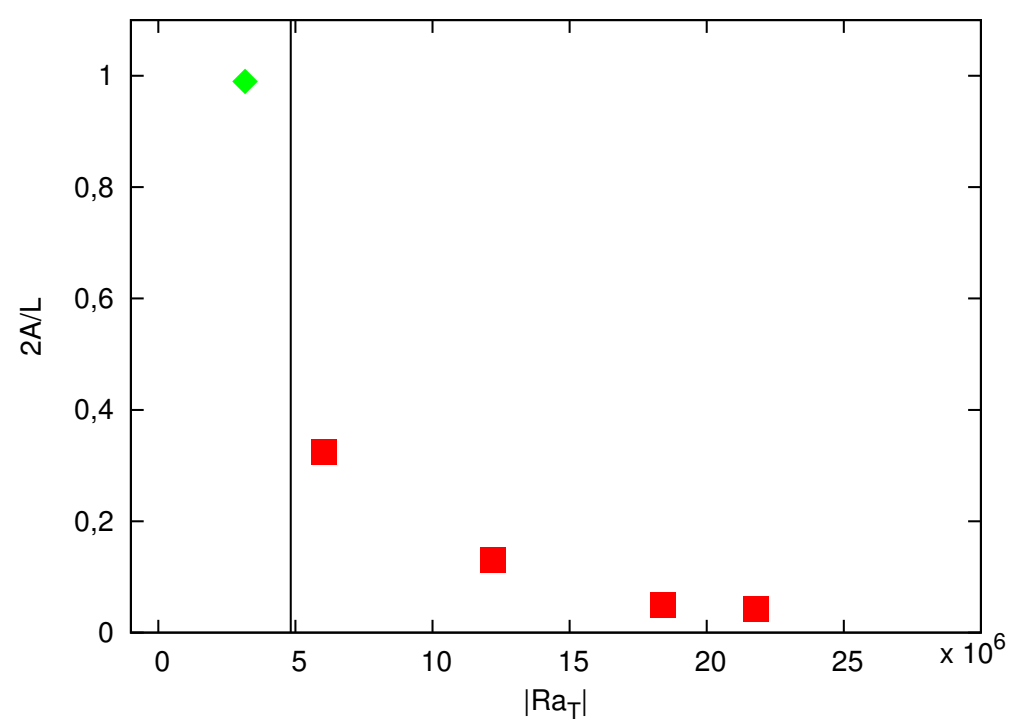

AвB. 3.13: maximale und minimale Höhe der Isotherme $T=0,51$ für $R a_{c}=2,92 \cdot 10^{10}$ als Funktion von $\left|R a_{T}\right|$ (rot). Eine Messung wurde im konvektiven Regime gemacht. Da hier kein Temperaturgradient außerhalb der Randschichten existiert, wurde dieser Punkt auf $2 A / L=1$ gesetzt (grün). Die Senkrechte Linie kennzeichnet den Übergang.

nachbarten Fingern auszutauschen, Ionen aber nicht ausgetauscht werden können, da sie zu langsam diffundieren. So kann man annehmen, dass Finger sich nur bilden können, wenn die Wärme mindestens eine Fingerdicke innerhalb einer Transitzeit von einer Platte zur anderen diffundiert. Das Verhältnis der Diffusionslänge zur Fingerdicke $\frac{1}{d} \sqrt{\kappa_{T} L / v}$ sollte daher mindestens 1 sein. Allerdings lässt sich hier nicht direkt vorhersagen, ab wann die Finger nicht mehr existieren können. In Tabelle 3.2 ist dies für den Übergang aufgelistet. Man erkennt, dass die Werte alle etwas über 1 sind (bis auf eine Ausnahme). Da man, wie in Abschnitt 3.3.2 gezeigt, weiß, dass die Geschwindigkeit mit abnehmendem Auftriebsverhältnis steigt, so sinkt das Verhältnis der Diffusionslänge zur Fingerdicke. Setzt man $\frac{1}{d} \sqrt{\kappa_{T} L / v}=1$ als Übergangskriterium, so ergibt sich:

$$
\left|R a_{T}\right|=6,6 \cdot 10^{-6} \operatorname{Pr}^{6 / 7} \operatorname{Ra}_{c}^{22 / 21} .
$$

$22 / 21=1,05$ und liegt noch im Fehlerintervall des Fits von Gleichung 3.7. In Abbildung 3.14 sind noch einmal der beste Fit der Datenpunkte, der Fit mit festgesetztem Exponenten von 1 und der Fit mit festgesetztem Exponenten von 22/21 aufgetragen. Man erkennt, dass alle drei Funktionen die Daten gut beschreiben und kaum voneinander zu unterscheiden sind. Es sind daher weitere Messungen, vor allem bei verschiedenen Prandtl- und 


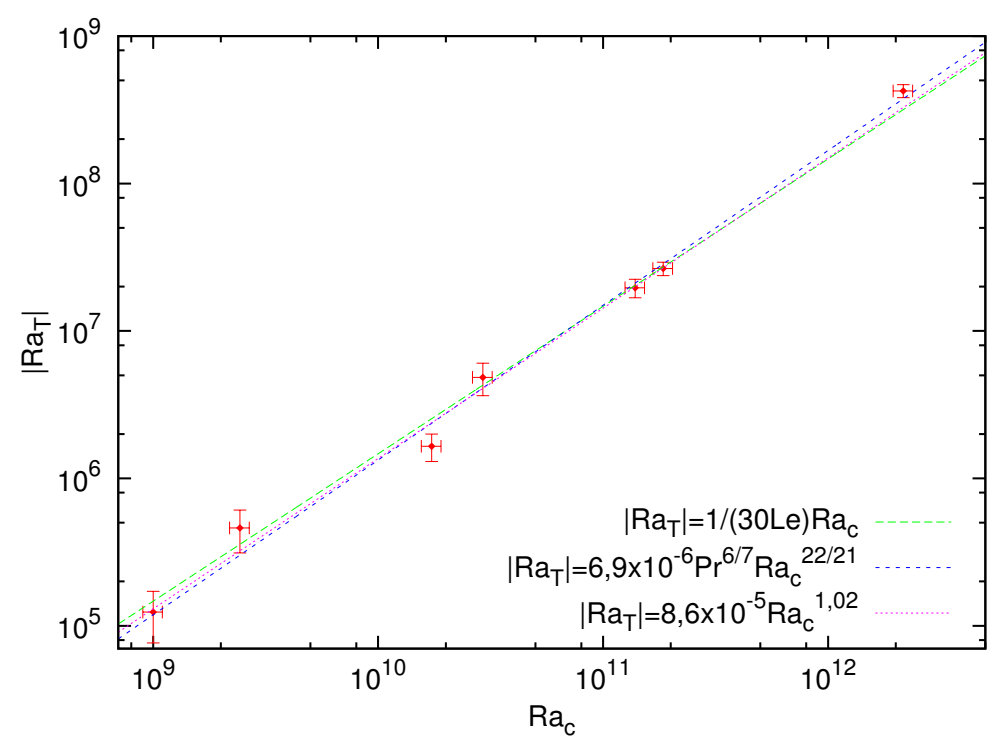

Aвв. 3.14: Die magenta farbene Linie ist die gleiche wie in Abbildung 3.2 und stellt den besten Fit der Daten (rote Punkte) dar. Die blaue Linie stellt einen Fit an die Datenpunkte nach Gleichung 3.11 dar, die grüne Linie einen Fit für festes $|\Lambda|=1 / 30$.

Schmidtzahlen zu machen, da die Kriterien unterschiedliche Abhängigkeiten von diesen beiden Parametern haben $\left(|\Lambda|=\right.$ const und $\left.|\Lambda| \sim \operatorname{Pr}^{6 / 7} R a_{c}^{1 / 21} L e\right)$. [Müller, 2013] hat schon gezeigt, das bei geringerer Lewiszahl der Übergang bei $|\Lambda|=1 / 6$ für $R a_{c}>2 \cdot 10^{5}$ $\mathrm{zu}$ erwarten ist.

[Yang et al., 2015a] haben basierend auf [Kellner and Tilgner, 2014] numerische Untersuchungen des Überganges, vor allem in Abhängigkeit von $L e^{6}$, gemacht. Ihre Berechnungen sind für $L e=1,10$ und 100 und für $R a_{c}=10^{7}, 10^{8}$ und $10^{9}$. Zwar ist die Auflösung ihrer Studie am Übergang sehr grob, trotzdem konnten sie für $L e \approx 10^{2}$ einen Übergang vom konvektiven zum Fingerregime bei $|\Lambda| \approx 1 / 30$ finden und für $L e \approx 10$ bei $|\Lambda| \approx 1 / 6$. Des Weiteren fanden sie bei konstantem $L e=100$ heraus, dass der Übergang bei konstantem $|\Lambda|$ stattfindet, d.h. unabhängig von der chemischen Rayleighzahl ist. Dies unterstützt die Ergebnisse dieser Arbeit. Jedoch ist anzumerken, dass sie für die Abhängigkeit des Überganges $|\Lambda|(L e)$ einen antiproportionalen Zusammenhang gefunden haben, da für kleinere Le der Betrag des Auftriebsverhältnisses am Übergang steigt. Dies würde allerdings für $L e<227$ widerlegen, dass Gleichung 3.11 ein Kriterium für den Übergang

\footnotetext{
${ }^{6}$ Dabei wurde $\operatorname{Pr}$ festgehalten und $S c$ variiert.
} 
ist, da nach dieser Gleichung $|\Lambda| \sim \operatorname{Pr}^{6 / 7} R a_{c}^{1 / 21} L e$ für den Übergang gelten müsste. Für $L e>227$ kann erst einmal dafür keine Aussage getroffen werden, da der antiproportionale Zusammenhang von Übergang und Le spätestens zusammenbricht, wenn die Diffusion der Temperatur nicht mehr ausreicht, um von einem Finger zum anderen zu diffundieren. Daher kann die Temperaturdiffusion immer noch eine mögliche Ursache für den Übergang bei großen $L e$ sein.

Trotzdem bleibt weiter offen, was den Übergang vom Fingerregime zum konvektiven Regime verursacht. 


\section{Thermohaline Treppen}

\subsection{Vorüberlegungen}

In allen Messungen aus Kapitel 3 wurden keine thermohalinen Treppen gefunden. Daher muss zuerst die Entstehung thermohaliner Treppen untersucht werden. Über dieses Thema streitet die Wissenschaft schon seit 50 Jahren, ohne auf einen geeigneten Konsens zu kommen. Bis heute gibt es daher sechs Modelle, die die Entstehung thermohaliner Treppen beschreiben. Im Folgenden werden dieses Modelle vorgestellt:

- kollektive Instabilität [Stern, 1969]

Wenn Finger in einem ungebundenen Medium aufgrund von Temperatur- und Salzgradienten wachsen, werden diese anfällig für lange interne Wellen. Durch die Auslenkung der Finger durch diese Wellen kommt es zur lokalen Erhöhung des Dichtegradienten an einigen Stellen und zur lokalen Verringerung an anderen. Dies führt zu einer Änderung der Temperatur- und Salzflüsse, die dafür sorgen, dass der Dichtefluss so verteilt wird, dass die daraus resultierende Auftriebskraft gegen die Stromrichtung der Finger wirkt. Solch ein System ist anfällig für Instabilitäten, wenn für die Stern-Zahl $A$

$$
A=\frac{\alpha Q-\alpha_{c} j}{\nu\left(\alpha \delta_{y} T-\alpha_{c} \delta_{y} c\right)}>1
$$

gilt $^{1}$. Denn dann ist die Auftriebskraft der Finger stärker als die viskose Dämpfung und die Amplitude der Welle steigt und sorgt für ein Aufbrechen der Finger in thermohaline Treppen. Zwar gilt in vielen Bereichen des Ozeans mit thermohalinen Treppen $A>1$, aber schon in Experimenten mit z.B. Salz und Zucker wurden Sternzahlen von $A \approx 10^{-3}$ und thermohalinen Treppen gefunden (vgl. u.a. [Lambert and Demenkow, 1988]).

\footnotetext{
${ }^{1} \delta_{y}$ ist der Gradient in $y$-Richtung.
} 
- metastabile Gleichgewichte, die durch externe Störungen entstanden sind [Stern and Turner, 1969]

Externe Störungen können dafür sorgen, dass sich die Gradienten für Temperatur und Salz ändern. Dadurch kann es passieren, dass das System in die typische Stufenform gezwungen wird. Da dieser Zustand als metastabil betrachtet wird, kann das System dort lange verweilen. Für thermohaline Treppen im diffusiven Regime konnte nachgewiesen werden, dass diese Störungen notwendig sind, um Treppen zu erzeugen. Für das Fingerregime hingegen sind sie hilfreich, aber nicht notwendig.

- negative Dichtediffusion [Schmitt, 1994]

Doppelt diffusive Konvektion wird durch das Freisetzen potentieller Energie der instabilen Komponente angetrieben. Dabei wird der obere weniger dichte Bereich leichter und der untere dichtere Bereich schwerer. Somit ist der Dichtefluss dem Dichtegradienten entgegengesetzt. Solche Systeme sind instabil. Allerdings gilt das für alle doppelt diffusiven Systeme mit $|\Lambda|>1$. Für den Wärme-Salz-Fall können sich thermohaline Treppen nur bis $|\Lambda|<2$ bilden. Dies entspricht etwa $2 \%$ des Parameterraumes.

- Instabilitäten der Gesetzmäßigkeiten der Flussgradienten [Radko, 2003]

Das Flussverhältnis $\gamma=\frac{\alpha_{T} Q}{\alpha_{c} j}$ ist keine monotone Funktion von $|\Lambda|$. Für $|\Lambda|>1$ fällt $\gamma$ bis zu einem Minimum und steigt danach wieder an. Das Minimum ist im Ozean bei $|\Lambda| \approx 2$. Wenn eine Störung im Temperaturfeld größer ist als die Störung im Salzfeld, wird das lokale Auftriebsverhältnis am Ort des größten Temperaturgradienten (bei $y=\pi / m)^{2}$ maximal. Solange $\gamma$ mit steigendem $|\Lambda|$ kleiner wird, steigt $\gamma$ an diesem Ort und sinkt bei $y=0$ und $y=2 \pi / m$. Dadurch kommt es zu einer Ansammlung von Wärme relativ zum Salz. Dies führt zu einem Anstieg von $|\Lambda|$ bei $y=0$ und $y=2 \pi / m$ und damit zu einem Absinken von $\gamma$ an diesen Orten. Bei $y=\pi / m \operatorname{sinkt}|\Lambda|$ und $\gamma$ steigt weiter an. Dieser Mechanismus sorgt für ein immer weiteres Anwachsen der Störung. Dadurch kommt es zu lokalen instabilen Dichteschichtungen. Diese Regionen beginnen dann zu konvektieren und bilden Konvektionszonen, die die Finger zwischen sich einschließen. Da der Dichtetransport in den Fingern entgegen des Gradienten ist, also nach unten, werden die Konvektionsbereiche durch die Finger mit "Dichte" versorgt. Von der oberen Konvektionszone wird "Dichte" abgesaugt und durch die Finger zur unteren Konvektionszone gebracht. Dort treibt die "Dichte" die untere Konvektionszone an. Dadurch befindet sich das System wieder in einem stabilen Zustand, der thermo-

\footnotetext{
${ }^{2} m$ ist die Wellenzahl der Störung.
} 
halinen Treppe. Da das Minimum von $\gamma(|\Lambda|)$ für das Wärme-Salz-System bei 2 liegt, können sich thermohaline Treppen nur im Bereich von $|\Lambda|<2$ bilden. Dies deckt sich mit den Beobachtungen im Ozean. Die Größe der entstehenden Treppenstufen liegen im Ozeanfall etwa eine Größenordnung unter den Beobachtungen. Schaut man sich z.B. die Messungen von [Zodiatis and Gasparini, 1996] oder das Experiment von [Krishnamurti, 2003] an, sieht man, dass die Stufenhöhen mit der Zeit anwachsen, d.h. einige Stufen verschmelzen mit anderen zu größeren Strukturen. Dies könnte die Diskrepanz vom Modell zu den Beobachtungen schließen. Zuerst bilden sich thermohaline Treppen mit kleinen Stufen, die dann mit der Zeit zu großen Stufen verschmelzen.

\section{- Sonstige}

Angewandte Flussmechanismen [Turner and Stommel, 1964] und thermohaline Intrusionen [Merryfield, 2000] werden in diesem Kontext nicht betrachtet, da ersteres nur für das diffusive Regime wichtig ist und letzteres horizontale Intrusionen braucht, die in den Experimenten dieser Arbeit nicht vorhanden sind.

Experimentell wurden solche thermohalinen Treppen bereits im Labor erzeugt. Ein paar Beispiele werden als nächstes vorgestellt.

Platziert man warmes Wasser über kaltem und rührt die Bereiche seperat um, so entsteht eine scharfe Grenze zwischen dem warmen und kalten Wasser. Durch Hinzufügen von Salz in das warme Wasser wachsen Finger vom Interface aus bis sie eine bestimmte Länge erreichen und Konvektionswalzen antreiben. Solch ein experimenteller Aufbau ist ein sogenanntes "run-down"-Experiment, d.h. die Gradienten in $T$ und $S$ ändern sich kontinuierlich mit der Zeit. [Turner, 1967] hat solch eine Technik für Versuche mit Wärme und Salz in Wasser benutzt.

Eine weitere Methode ist es, eine horizontale Barriere in die Messzelle zu legen. Unter der Barriere befindet sich kaltes, frisches Wasser und über der Barriere warmes salziges Wasser, welches etwas weniger dicht ist als das Wasser unter der Barriere. Nun wird diese vorsichtig entfernt und es bilden sich wieder Finger von der Grenzfläche aus, die bei Erreichen einer spezifischen Länge Konvektionswalzen antreiben. Auch diese Technik ist ein "run-down"-Experiment und wird die "dam break"-Methode genannt. [Stern and Turner, 1969] haben diese Methode im Wärme-Salz-System und Salz-ZuckerSystem benutzt. [Linden, 1973] nutze diese Technik für Zucker-Wärme-Systeme.

Um die Fingerbildung abseits von starken Gradienten zu untersuchen, kann man lineare Gradienten für $T$ und $S$ erzeugen, und dann die Fingerbildung beobachten, auch hier bilden sich Finger, die zu thermohalinen Treppen zerfallen. Diese Technik kann man 
als "run-down"-Experimente durchführen, oder durch Erhalt der Randbedingungen an der oberen und unteren Platte endlos durchführen. [Krishnamurti, 2003] hat dies für ein Zucker-Salz-System durchgeführt.

Ein ähnlicher Versuchsaufbau wie von [Krishnamurti, 2003], nur für Wärme und Kupferionen, wurde für die zweite Messreihe dieser Arbeit benutzt.

\subsection{Das Experiment}
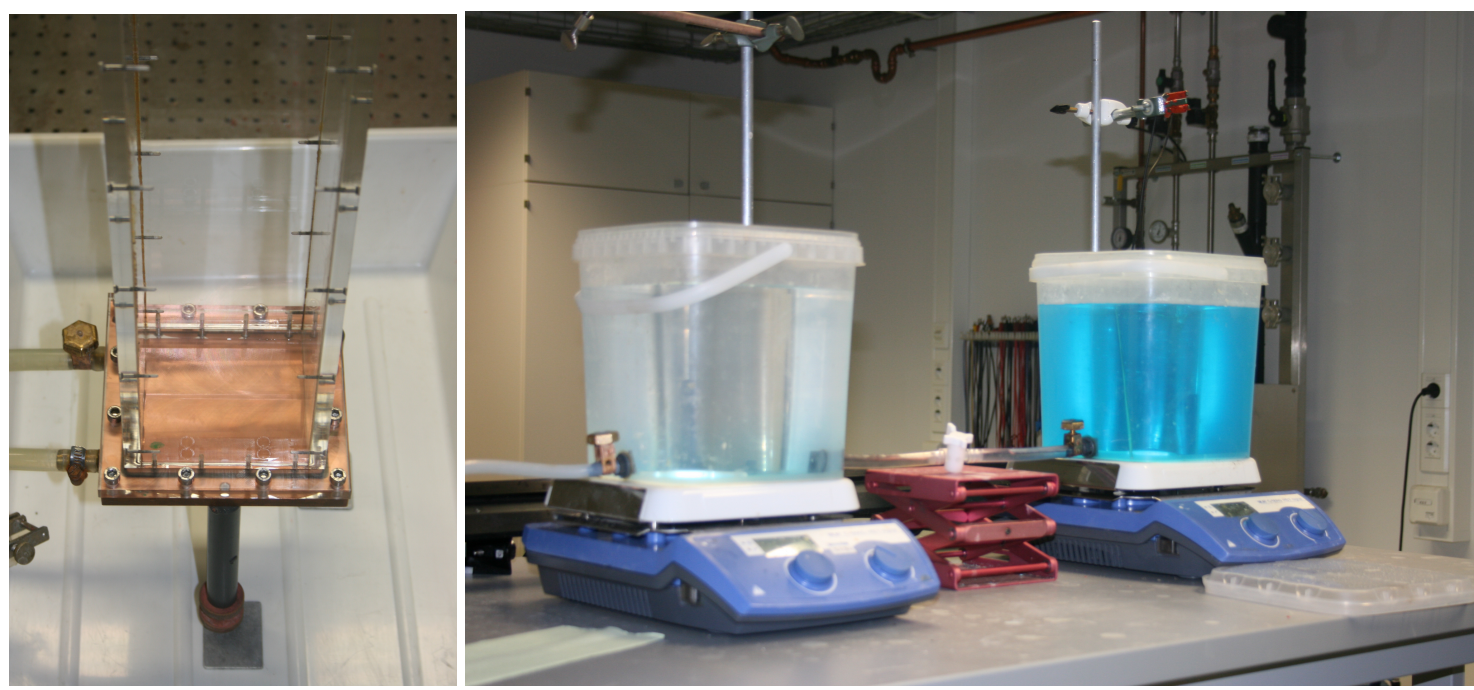

ABB. 4.1: LINKS: Bodenplatte und Plexiglasseitenwände des Versuches zur Untersuchung thermohaliner Treppen

RECHTS: Zwei Eimer auf Magnetrührern zum Befüllen der Zelle über die Zwei-Eimer-Methode.

Für dieses Kapitel wurde der Versuchsaufbau aus Kapitel 3.2 abgeändert. Als Boden und Deckel dienen immer noch zwei Kupferplatten. Ihre Maße wurden auf $15 \mathrm{~cm}$ mal $15 \mathrm{~cm}$ halbiert. Die Seitenflächen sind wieder aus Plexiglas, aber diesmal mit einer Höhe von 40 cm bzw. $80 \mathrm{~cm}$. Der Dichtungsring ist diesmal im Plexiglas eingearbeitet, sodass die Seitenwände nur auf die Kupferplatten aufgeschraubt werden müssen (vgl. Abb. 4.1 links). Die Fläche innerhalb der Plexiglaswände beträgt $10 \mathrm{~cm}$ mal $10 \mathrm{~cm}$. Durch zwei Thermostate können die Platten wieder auf eine spezifische Temperatur gebracht werden. Über eine Spannungsquelle kann wieder eine Spannung wie im Kapitel 2.3 beschrieben angelegt werden. Aufgrund der Größe der Zelle wurde das Plateau diesmal anders bestimmt. In der Zelle wurde ein linearer stabiler Temperaturgradient erzeugt. Dann wurde die Span- 
nung langsam erhöht, bis sich an den Platten Blasen bildeten (Beginn von Bereich III in Abbildung 2.3). Danach wurde die Spannung soweit verringert bis gerade keine Blasen mehr entstehen (oberer Bereich von Bereich II in Abbildung 2.3).

Auch die Art der Befüllung wurde geändert. In Kapitel 3 wurde die Zelle zuerst gefüllt und dann durch Diffusion ein stabiler linearer Temperaturgradient eingestellt. Dies dauerte bei der Zelle mit $2 \mathrm{~cm}$ Höhe 53 Minuten, bei der Zelle mit $20 \mathrm{~cm}$ Höhe 90 Stunden. Für die neuen Höhen hätte man 15 Tage bzw. 60 Tage warten müssen. Daher wurde die Zwei-Eimer-Methode (vgl. [Oster, 1965] mit Modifikationen nach [Maxworthy, 1972]) benutzt.

In Tank I (vgl. Abb. 4.2) befindet sich das Fluid mit Konzentration $c=0$ und Temperatur $T=T_{\text {unten }}$. Dieser ist über ein Rohr mit Tank II verbunden $\left(T=T_{\text {oben }}\right.$ und $c=2 c_{0}$ ). Beide Tanks werden über Magnetrührer gut durchmischt. Von Tank I aus kann man das Fluid über ein Rohr, welches auf einem Schwimmer endet, in die Zelle ablassen. Dadurch fliefst Fluid aus Tank II in Tank I nach und ändert damit die Konzentration und Temperatur in Tank I. Lässt man das Fluid gleichmäßig aus Tank I ausströmen, entsteht dort ein lineares Temperaturprofil mit $T=T_{\text {unten }}$ an der unteren Platte und $T=T_{\text {oben }}$ an der oberen Platte. Das Konzentrationsprofil in der Zelle ist ebenfalls linear mit $c=0$ an der unteren Platte und $c=2 c_{0}$ an der oberen. Die Abweichungen vom linearen Profil sind dabei gering und hängen vor allem von der Geschwindigkeit des Abflusses aus Tank I ab. (vgl. [Zurigat et al., 1990] und [Ghajar and Bang, 1993]). Allerdings lässt sich damit nur Fluid mit einer Dichteschichtung von $|\Lambda|>1$ erzeugen, da sonst das neue schwerere Fluid nach unten sinken würde, bevor sich Strukturen bilden könnten, die dem entgegen wirken. Die Befüllungsdauer der großen Zelle $(80 \mathrm{~cm})$ beträgt damit ca. 2 Stunden.

Für $|\Lambda|<1$ muss das Verfahren soweit abgeändert werden, dass nur die Temperatur geschichtet wird. Daher ist im Tank I Fluid mit $T=T_{\text {unten }}$ und $c=c_{0}$ und im Tank II Fluid mit $T=T_{\text {oben }}$ und $c=c_{0}$. Ein linear stabil geschichteter Temperaturgradient bildet sich dadurch aus. Das Konzentrationsprofil ist uniform. Wie in Kapitel 3.3.1 schon erwähnt hat dies keinen Einfluss auf die Strukturen, die sich am Ende bilden, sondern nur auf die Zeitdauer, bis sie sich bilden. Diese liegt bei etwa 12 Stunden. Es wurde auch eine Messung ${ }^{3}$ ohne Gradient als Startbedingung durchgeführt. Diese brauchte dann fünf Tage bis sich die Treppe ausgebildet hatte.

\footnotetext{
$\overline{{ }^{3} \Lambda=-0,5 ; R a_{T}=-1,82 \cdot 10^{10} ; R a_{c}=7}, 08 \cdot 10^{12}$
} 


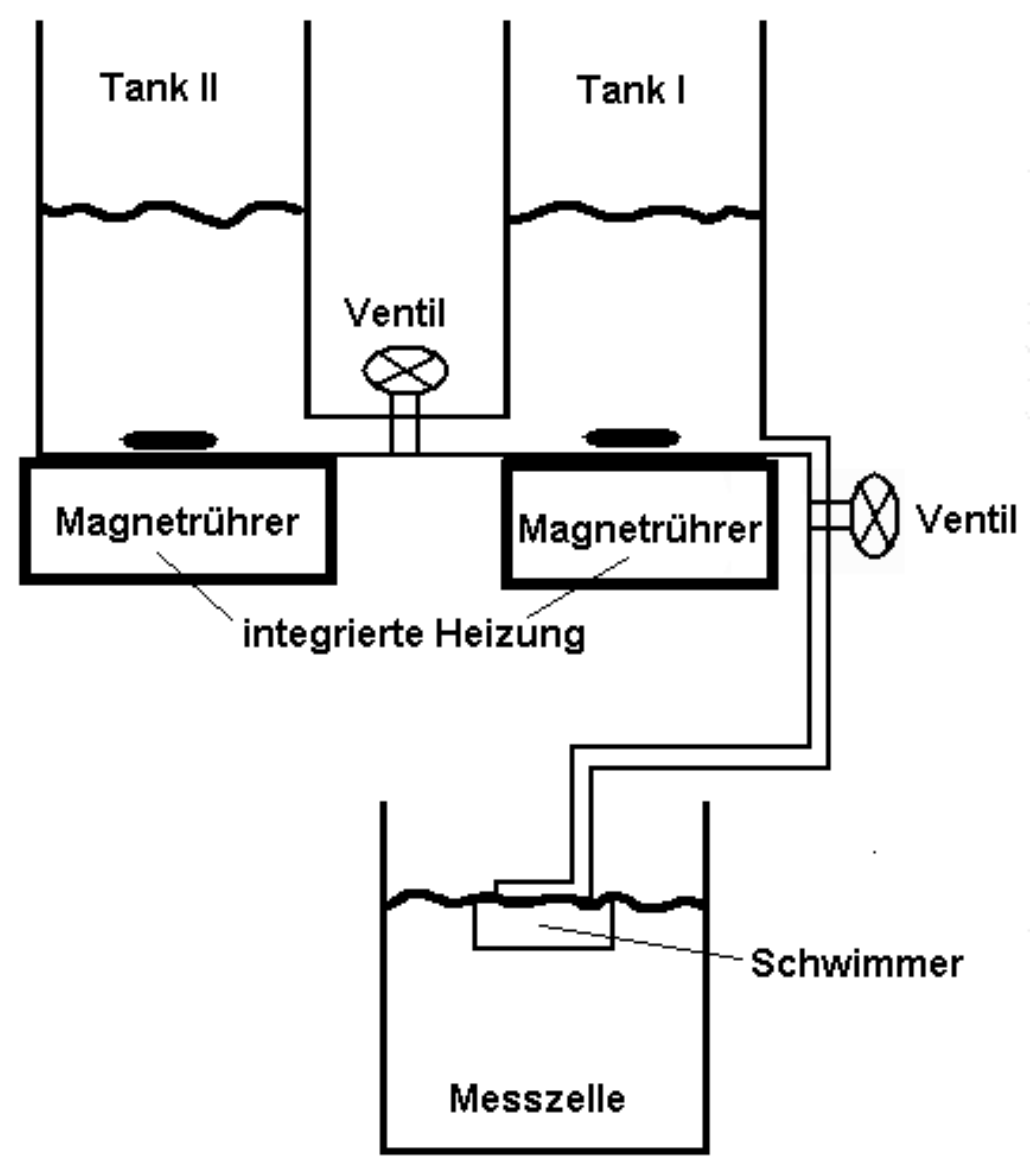

ABB. 4.2: Schematische Darstellung der modifizierten Zwei-Eimer-Methode, um lineare Gradienten zu erzeugen. 


\subsection{Ergebnisse}

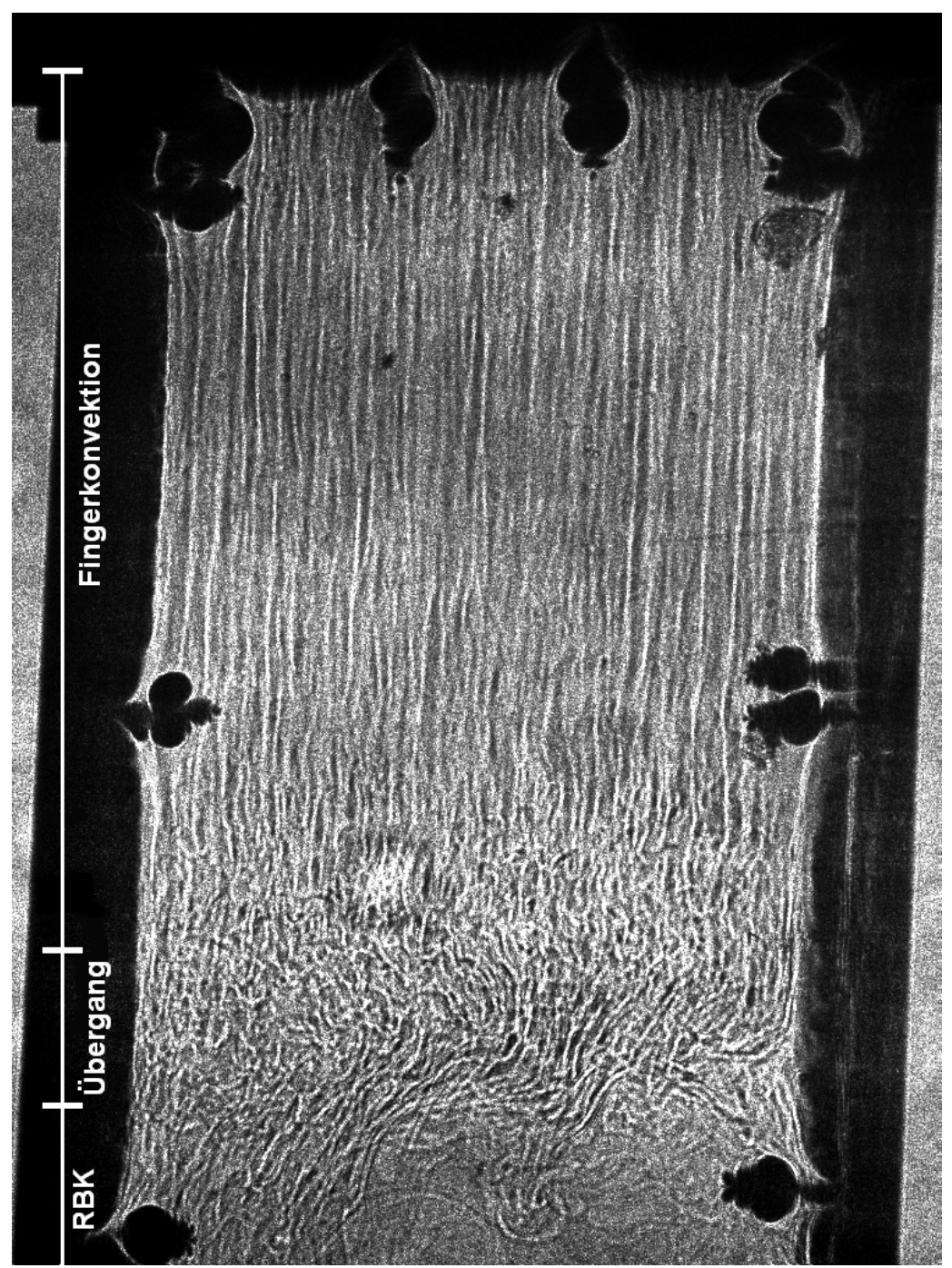

ABB. 4.3: Shadowgraphaufnahme des oberen Fingerbereiches einer thermohalinen Treppe bei einem Auftriebsverhältnis von $|\Lambda|=1$. Man erkennt gut die Finger, den Übergang und den Bereich mit Rayleigh-Bénard-Konvektion. Die Finger haben eine Länge von $0,138 \mathrm{~m}$.

Mit diesem Versuchsaufbau ist es nun gelungen, thermohaline Treppen zu erzeugen. Dabei sind in 13 von 15 Messungen Treppen entstanden, die aus zwei Fingerbereichen an den Platten und einem Konvektionsbereich in der Mitte bestehen. Bei den zwei übrigen Messungen hat sich nur ein Fingerbereich an der oberen Platte gebildet und darunter ein Konvektionsbereich. Wahrscheinlich wurde bei diesen zwei Messungen der untere Teil der 
Zelle zu schnell befüllt, sodass dort durch Vermischung kein Gradient vorherrschte. Dies würde bedeuten, dass sich die Finger dort zum Zeitpunkt der Messung noch nicht ausgebildet hatten. Bei dem in Kapitel 4.2 beschriebenen Testversuch brauchte das System fünf Tage bis es den statistisch-stationären Zustand erreicht hatte. Um dies zu verifizieren, wurde eine Messung durchgeführt, bei der sich nur ein Fingerbereich gebildet hatte. Nach fünf Tagen hatte sich hier ein zweites Fingerregime an der anderen Platte gebildet. Für die beiden Zellen mit $\Gamma=1 / 4$ und $1 / 8$ kann somit eine Endkonstellation von zwei Fingerbereichen an den Platten und dazwischen ein Konvektionsbereich angenommen werden. Die zwei Messungen mit nur einem Fingerbereich sind trotzdem mit Vorsicht zu betrachten. Die Randbedingungen für die Finger werden zum einem durch die Elektrode und zum anderen durch die Konvektion bestimmt. Diese hängt aber u.a. vom Aspektverhältnis $\Gamma$ und den Rayleighzahlen ab (vgl. u.a. [Krishnamurti, 1995]), die mit der Höhe variieren. Allerdings fallen die Eigenschaften der Finger an der oberen Platte nicht aus dem Rahmen der anderen Messwerte mit zwei Fingerzonen.

In Abbildung 4.3 ist eine Treppenstufe mit Fingerregime, Übergangsbereich und RayleighBénard-Zone dargestellt.

Um die genaue Länge $L$ der Finger zu bestimmen wurde ein Geschwindigkeitsprofil, wie in Abbildung 4.4 beispielhaft gezeigt, gemessen. Dabei wurde die Geschwindigkeit horizontal über alle $x$ und $t$ gemittelt. Man erkennt im Fingerregime den typischen Verlauf mit geringer x-Komponente der Geschwindigkeit. Diese steigt langsam in Richtung Übergang an. Dieser Anstieg kommt von internen Wellen in den Fingern. Diese sind deutlich in den Shadowgraph und PiV-Aufnahmen zu erkennen. Die Amplitude dieser Wellen beträgt etwa eine Fingerbreite und sie haben eine Periode von ca. 80 s. Sie haben die größte Amplitude am Übergang, wo sie durch eintreffende Plumes entstehen und nehmen in Intensität mit Annäherung zur Platte ab. Diese Wellen sind in allen Messungen aufgetaucht, aber nicht immer vorhanden, sodass es Zeiträume während einer Messung mit Wellen und Zeiträume ohne gab. Diese Wellen wurden im Zuge dieser Arbeit nicht weiter betrachtet.

Am Übergang steigt die $\mathrm{x}$-Komponente der Geschwindigkeit stark an, hat ihr Maximum im oberen Rayleigh-Bénard-Bereich und sinkt im Inneren wieder ab. Dies ist ein eindeutiges Indiz für die Konvektionswalze. Die y-Komponente steigt am Übergang auch stark an, bleibt aber im Inneren auf einem hohen Niveau. Da die Lage der Konvektionswalze nicht zwingend parallel zu der 2D-PiV-Ebene liegt, kann die Geschwindigkeit in der Konvektionswalze auch höher sein. Am Profil ändert dies aber nicht viel. Die Aufnahmezeit, 


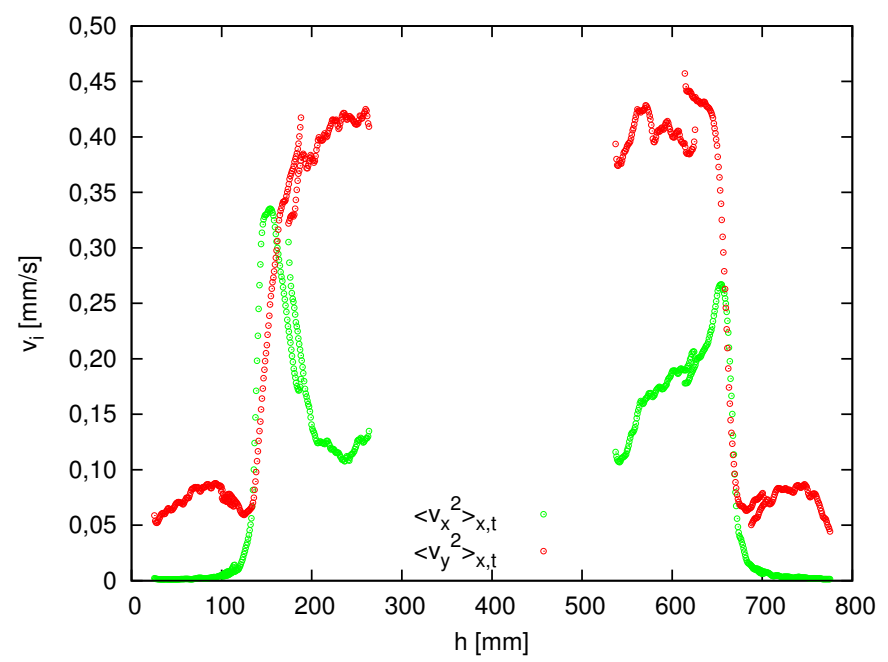

AвB. 4.4: Geschwindigkeitsprofil für $R a_{c}=1,13 \cdot 10^{14}$ und $\left|R a_{T}\right|=6,36 \cdot 10^{11}$. Die Zelle erstreckte sich von 0 bis $800 \mathrm{~mm}$. Die horizontal und zeitlich gemittelte $\mathrm{x}$ Komponente der Geschwindigkeit ist wie erwartet gering im Fingerbereich, und ca. gleich groß wie die y-Komponente im Rayleigh-Bénard-Bereich. Auffällig ist die große Geschwindigkeitsgrenzschicht in der y-Komponente im Fingerbereich. Die Länge der Finger $L$ wird mit dem Ort der minimalen y-Geschwindigkeit am Übergang definiert.

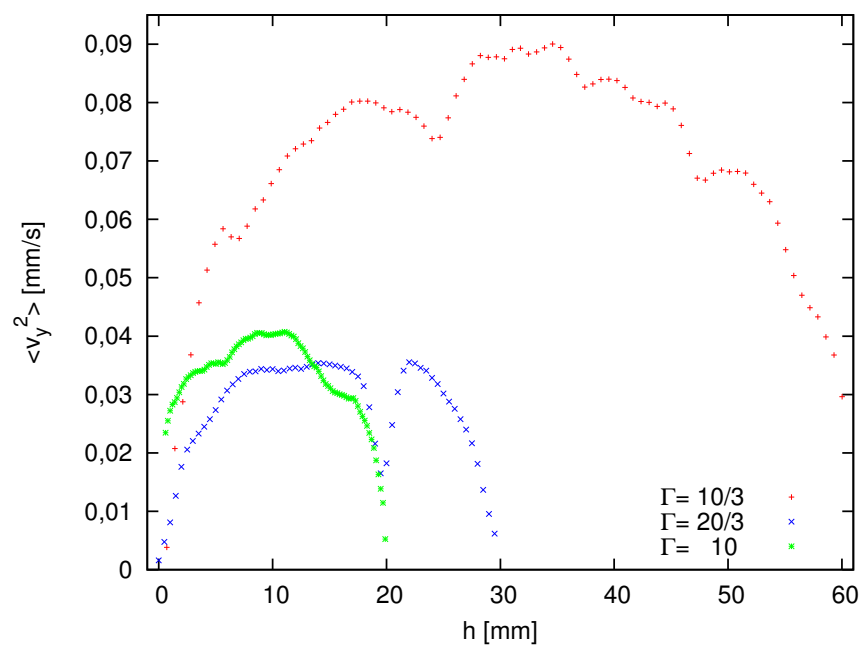

AвB. 4.5: Die horizontal und zeitlich gemittelte y-Komponente der Geschwindigkeit für drei Messungen aus Kapitel 3. Auch hier ist die Geschwindigkeitsgrenzschicht im Vergleich zur gesamten Fingerlänge größer als erwartet. rot: $R a_{c}=2,92$. $10^{10}$ und $\left|R a_{T}\right|=2,72 \cdot 10^{6}$; blau: $R a_{c}=2,43 \cdot 10^{9}$ und $\left|R a_{T}\right|=4,05 \cdot 10^{6}$; grün: $R a_{c}=1,00 \cdot 10^{9}$ und $\left|R a_{T}\right|=6,81 \cdot 10^{5}$ 
über die gemittelt wurde, betrug in den Fingern 4 mal 2 Minuten, am Übergang und im Konvektionsbereich je 12 mal 2 Minuten ${ }^{4}$. Für die Finger ist dies ausreichend, da ihre zeitliche Variation nur gering ist ${ }^{5}$. Im Konvektionsbereich reicht diese Zeit aus, um ein qualitatives Bild zu erhalten. Bedenkt man aber, dass die Konvektionswalze für eine Umdrehung ca. 40 Minuten braucht, so hätte man die Aufnahmezeit noch verlängern müssen.

Auffällig ist noch der Verlauf der y-Komponente im Fingerbereich. Man erkennt eine relativ große Geschwindigkeitsrandschicht, die etwa $L / 4$ beträgt. Dies war nicht zu erwarten. In Abbildung 4.5 ist das Geschwindigkeitsprofil für drei Messungen aus Kapitel 3 gezeigt. Auch hier ist eine relativ große Geschwindigkeitsgrenzschicht zu erkennen. Eine Ursache konnte in dieser Messkampagne nicht gefunden werden. Problematisch ist dies, da die x-Komponente annähernd konstant bleibt und somit zur Erhaltung der Divergenzfreiheit die dritte Dimension ein entsprechendes Geschwindigkeitsprofil aufweisen muss. Dies ist nicht unplausibel, jedoch scheinen dann bei allen Messungen die Finger einen Unterschied zwischen den beiden horizontalen Dimensionen zu sehen. Am Übergang zur Konvektion kann dies durch die Konvektionswalze geschehen, an der Platte ist erst einmal nicht offensichtlich, woran das liegen könnte. Bei den Messungen zu Kapitel 3 kann man erkennen, dass $v_{x}$ manchmal am Rand, wie erwartet um $\vec{\nabla} \vec{v}=0$ zu erhalten, stark ansteigt. Das zeigt, dass für diese Zellen die beiden horizontalen Richtungen gleichwertig sind und es Zufall ist, welche Richtung eine erhöhte horizontale Geschwindigkeitskomponente hat.

Die Länge der Finger $L$ wurde als der Abstand definiert, der zwischen den beiden Minima der y-Komponente der Geschwindigkeit liegt. Das eine Minimum fällt mit der Platte zusammen, das andere ist an dem Ort, an dem die x-Komponente der Geschwindigkeit beginnt stark anzusteigen. Die Länge der Finger hängt von den Rayleighzahlen wie folgt ab:

$$
L / l_{y}=2,8 \cdot 10^{3} R a_{T}^{4 / 9} R a_{c}^{-3 / 4}
$$

Die ist in Abbildung 4.6 dargestellt. Die Finger werden mit zunehmendem stabilisierendem Einfluss der Temperatur länger und mit zunehmendem destabilisierendem Einfluss der Konzentration kleiner. Ab $L / l y=0,5$ liegt keine thermohaline Treppe mehr vor,

\footnotetext{
${ }^{4}$ Gemessen wurde 2 mal 2 Minuten, dann 10 Minuten Pause, dann 2 mal 2 Minuten, dann wieder 10 Minuten Pause und so weiter...

${ }^{5}$ Die größte zeitliche Änderung kommt von den Wellen.
} 


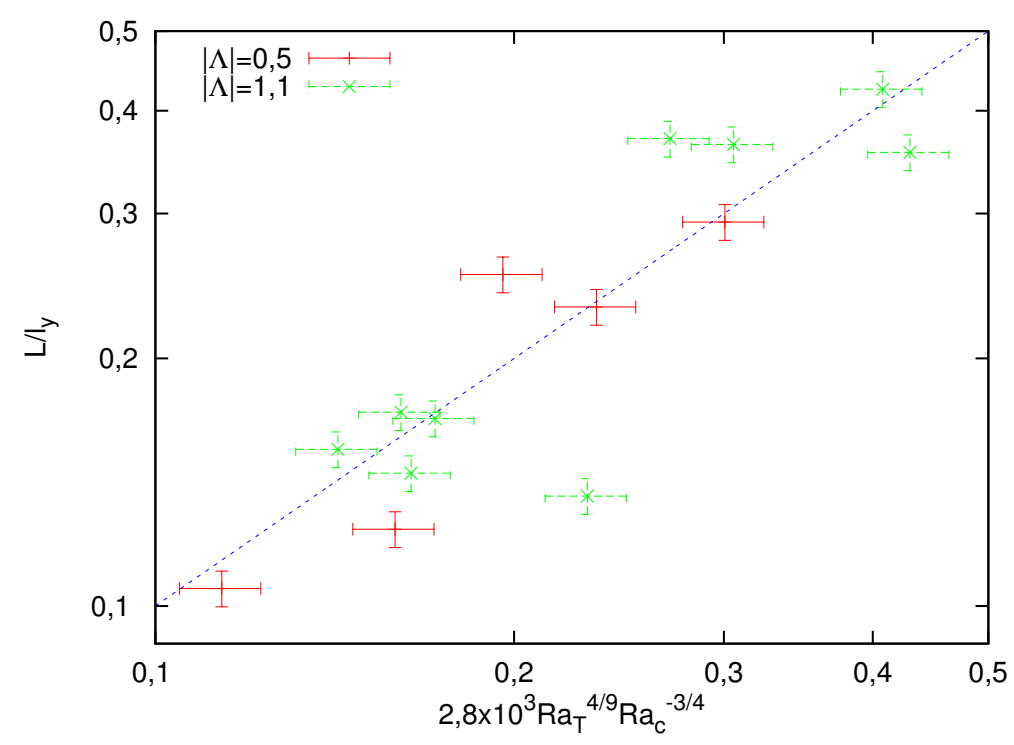

ABB. 4.6: $L / l y$ als Funktion von $R a_{c}$ und $R a_{T}$

sodass man eine Grenze für das Entstehen thermohaliner Treppen mit

$$
R a_{T}=3,68 \cdot 10^{-4} R a_{c}^{27 / 16}
$$

festlegen kann.

In Abbildung 4.7 ist die globale Sherwoodzahl gegen die globale chemische Rayleighzahl aufgetragen. Man erwartet ein analoges Verhalten zu Gleichung 2.13, da sowohl Sh von $j$ als auch $R a_{c}$ von $\Delta c$ linear abhängen. Der beste Fit durch die Daten ergibt

$$
S h=0,35 R a_{c}^{0,23} .
$$

Dies liegt nahe an den anderen experimentellen Daten aus Tabelle 2.1. Zum Vergleich sind mit der schwarzen Linie die Sherwoodzahlen markiert, die sich nach Gleichung 3.6 bei reiner Fingerströmung einstellen würden, d.h. die Zelle der Höhe $l_{y}$ hat Finger der Länge $L=l_{y}$. Nach [Krishnamurti, 2003] sollte die Sherwoodzahl um ca. einen Faktor 2 größer sein als bei der thermohalinen Treppe. Jedoch ergibt sich hier eine Abweichung zwischen Fingerströmung und Treppe von 3 bis 4 , abhängig von $R a_{c}$.

Das große Problem dieses Versuches ist es, dass die Konzentrationen und Temperaturen nur global und nicht lokal bekannt sind. Daher sind per se die Rayleighzahlen für den Fingerbereich nicht bekannt und ein Vergleich der Finger in einer solchen Treppe mit 


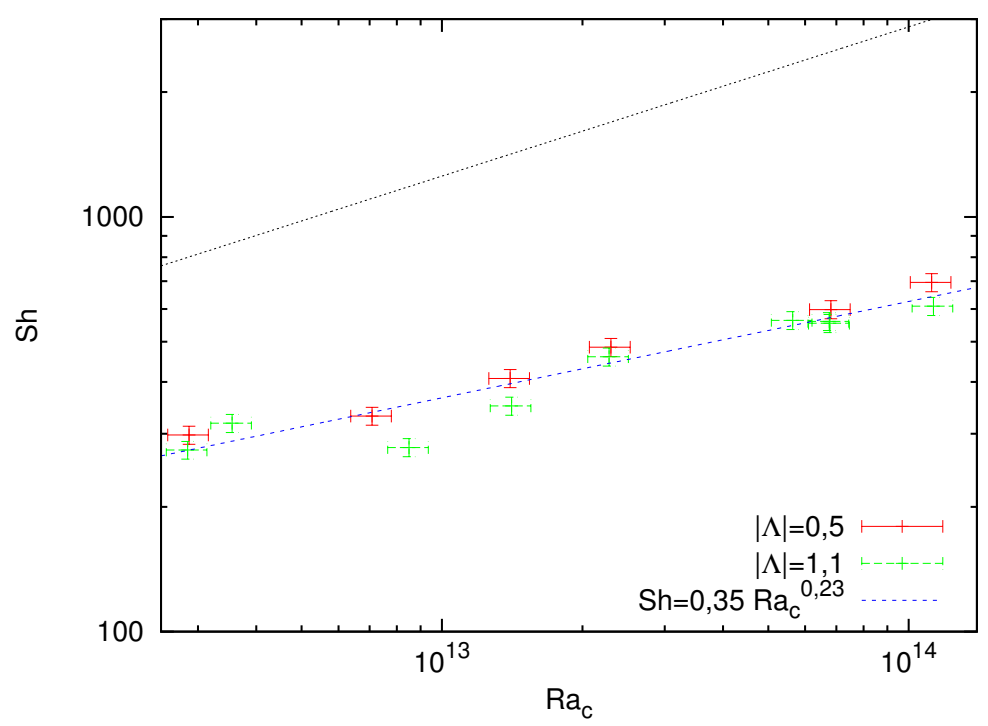

ABB. 4.7: $S h$ als Funktion von $R a_{c}$ über die gesamte thermohaline Treppe bestehend aus zwei Stufen. Die blaue Linie ist ein Fit an die Datenpunkte mit $S h=$ $0,35 R a_{c}^{0,23}$. Die schwarze Linie markiert die Sherwoodzahlen, die sich in reiner Fingerströmung mit Fingern der Länge $l_{y}$ nach Gleichung 3.6 einstellen würden.

z.B. den gebundenen Fingern aus Kapitel 3 oder [Hage and Tilgner, 2010] nicht möglich. Um dies doch noch tun zu können kann man Gleichungen 3.4, 3.5 und 3.6 hernehmen und umschreiben zu

$$
I \sim L^{-4} R e^{1,7}(d / L)^{-2,3}
$$

Diese Form ist unabhängig von $\Delta c$ und $\Delta T$. Der Strom durch die Zelle, die Geschwindigkeiten der Finger und ihre Dicke lassen sich relativ leicht bestimmen. Der Vorfaktor von Gleichung 4.5 ist erst einmal unbekannt und muss nicht der sein, der durch die Umformung entsteht.

In Abbildung 4.8 sind die Messwerte aus den Messungen und von [Hage and Tilgner, 2010] gegen Gleichung 4.5 aufgetragen. Die Streuung ihrer Messdaten ist relativ groß, da durch diese Auftragung die Fehler für $R e, d / L$ und $S h$ und die Abweichungen von den Gleichungen 3.4, 3.5 und 3.6 übertragen werden. Der Vorfaktor ergibt sich zu $3,9 \cdot 10^{-10}$. Die Daten aus dieser Arbeit haben einen anderen Vorfaktor. Dies ist nicht verwunderlich, da dieser u.a. von den Randbedingungen abhängt. Bei [Hage and Tilgner, 2010] waren die Finger zwischen zwei festen Rändern eingeschlossen, in dieser Arbeit gibt es einen festen Rand zu den Elektroden und einen freien zum Rayleigh-Bénard-Bereich. Verwunderlich 


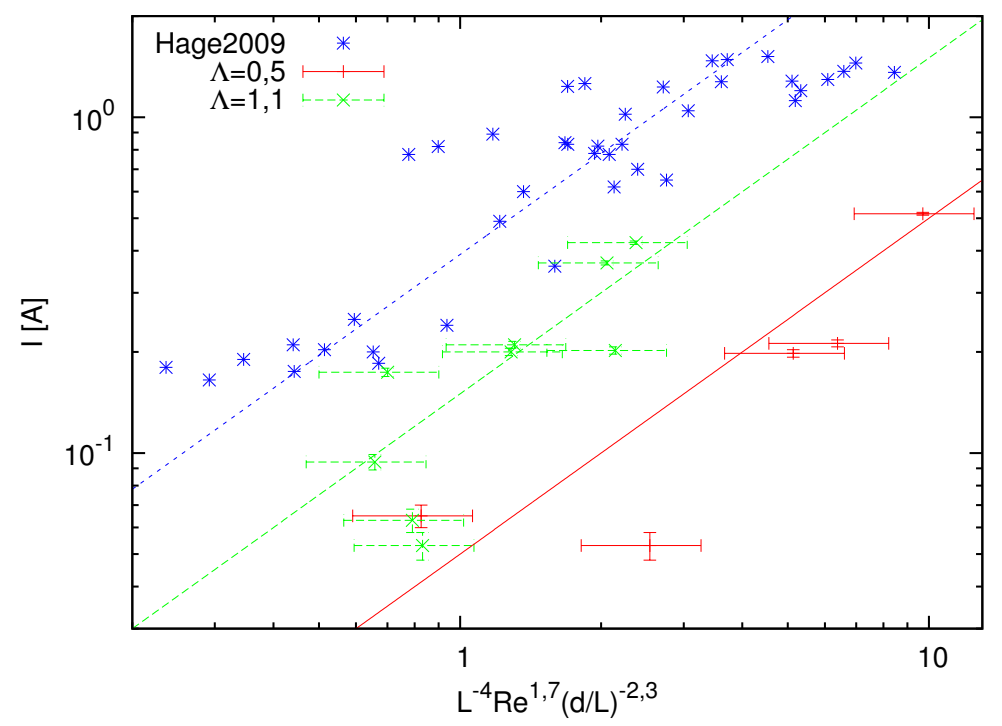

ABB. 4.8: Der Stromfluss durch die Zelle als Funktion von $L, R e$ und $d / L$ aus den Gleichungen 3.4, 3.5 und 3.6 gewonnen. Die Vorfaktoren sind: blau $\rightarrow 3,9 \cdot 10^{-10}$ für die Daten von [Hage, 2009]; grün $\rightarrow 5,0 \cdot 10^{-11}$ für $|\Lambda|=1,1$; $\operatorname{rot} \rightarrow 2,2 \cdot 10^{-11}$ für $|\Lambda|=0,5$. Der Unterschied in den Vorfaktoren lässt sich nur durch unterschiedliche Randbedingungen erklären. Diese sind bei den Daten von [Hage, 2009] offensichtlich, für die unterschiedlichen Auftriebsverhältnisse jedoch nicht.

ist nur, dass der Vorfaktor scheinbar vom Auftriebsverhältnis abhängt. Dies impliziert, dass die Randbedingungen unterschiedlich sein müssten. Nur eine Änderung der Strömung im Rayleigh-Bénard-Bereich kommt hierfür in Frage. In dieser Arbeit lässt sich für die beiden Auftriebsverhältnisse kein Unterschied erkennen.

Eine Abschätzung der Konzentration am Übergang zwischen Fingerströmung und RayleighBénard-Konvektion kann über letztere gemacht werden. Die Geschwindigkeiten in der Konvektionswalze sind durch die PiV-Aufnahmen bekannt. Nach [Hage, 2003], die Messungen für die gleichen Schmidt- und Prandtlzahlen gemacht hat, gilt:

$$
R e=1,7 \cdot 10^{-4} R a_{c}^{0,55} .
$$

Damit kann man die chemische Rayleighzahl des Konvektionsbereiches und damit das $\Delta c$, das mindestens nötig ist, um die Geschwindigkeiten zu erreichen, bestimmen. Man muss aber beachten, dass in dem System dieser Arbeit noch ein stabilisierender Tem- 
peraturgradient vorliegt. Dadurch bleibt dies nur eine Abschätzung für ein minimales $\Delta c$. Berechnet man nun diese Konzentrationsdifferenzen, stellt man fest, dass diese immer zwei Größenordnungen unter dem globalen $\Delta c$ liegen. Somit ist die Annahme, dass an der Grenze zwischen Fingerregime und konvektivem Regime eine Konzentration von $\Delta c / 2$ vorherrscht, nur mit einen Fehler von ca. $5 \%$ behaftet.

Der Temperaturgradient bleibt trotzdem erst einmal offen. Bekannt sind die Temperaturen somit nur an den Platten mit $T=T_{\text {oben }}$ und $T=T_{\text {unten }}$ sowie im konvektiven Bereich mit $T=\left(T_{\text {oben }}-T_{\text {unten }}\right) / 2$. Es wird für diese Arbeit erst einmal angenommen, dass die Temperaturgrenzschicht ebenfalls vernachlässigbar klein ist und somit eine Temperaturdifferenz von $\Delta T / 2$ über den Fingern herrscht.

Mit diesen Annahmen wurden die Rayleighzahlen für die Finger abgeschätzt und die Gültigkeit der Gleichungen 3.4, 3.5 und 3.6 überprüft. In den Abbildungen 4.9, 4.10 und 4.11 ist dies dargestellt. Die Vorfaktoren sind durch die geänderten Randbedingungen nicht mehr zwingend gleich und es ergibt sich:

$$
\begin{gathered}
d / L=1,21\left|R a_{T}\right|^{-1 / 3} R a_{c}^{1 / 9} \\
R e=3,3 \cdot 10^{-6}\left|R a_{T}\right|^{-1 / 2} R a_{c} \\
S h=0,0122\left|R a_{T}\right|^{-1 / 12} R a_{c}^{4 / 9}
\end{gathered}
$$

Die Abweichungen der Messwerte von den Gleichungen liegen in der Größenordnung der Abweichungen von [Hage and Tilgner, 2010], mit deren Werten die Gleichungen bestimmt wurden.

Daher scheint es nicht unplausibel, dass die Annahme von einem Konzentrationsgradienten von $\Delta c /(2 L)$ und einem Temperaturgradienten von $\Delta T /(2 L)$ über die Finger gerechtfertigt ist.

Der Massenfluss über ein Fingerinterface, welches von zwei Konvektionsbereichen umgeben ist, kann nach Gleichung 2.13 abgeschätzt werden. Dies wurde für die Annahme von einem Konzentrationsunterschied von $\Delta c / 2$ über die Finger in Abbildung 4.12 getestet. Die Datenpunkte zeigen wie erwartet eine leichte $\Lambda$-Abhängigkeit, die hier aber mit Messungen bei zwei verschiedenen $\Lambda$ nicht getestet werden konnte. Der Fit der Datenpunkte ergibt

$$
I=6,69 \cdot(\Delta c / 2)^{1,21}
$$

Dies stellt eine kleine Abweichung vom 4/3-Gesetz dar. Doch wie in Kapitel 2.2 schon erwähnt, wurden beim 4/3-Gesetz die Größe der Konvektionsbereiche und die Startbe- 
dingungen vernachlässigt. Der Exponent reiht sich gut in die Daten der anderen experimentellen Arbeiten aus Tabelle 2.1 ein.

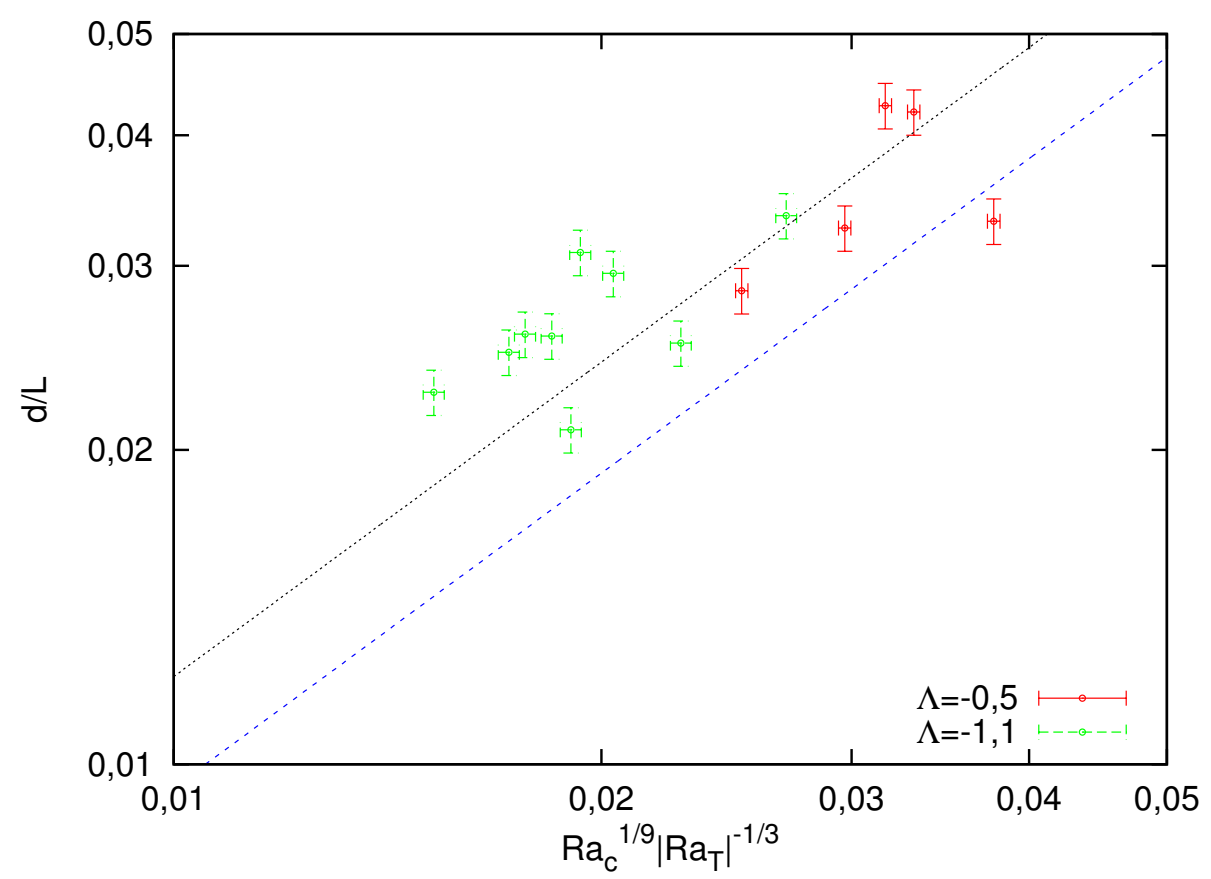

AвB. 4.9: Überprüfung der Gleichung 3.4 für die Finger in der thermohalinen Treppe unter der Annahme für einen Konzentrationsgradienten von $\Delta c /(2 L)$ und einen Temperaturgradienten von $\Delta T /(2 L)$. Die blaue Linie ist für den Vorfaktor 0,95, die schwarze für den Vorfaktor 1,21. Die Abweichung kommt durch die unterschiedlichen Randbedingungen. 


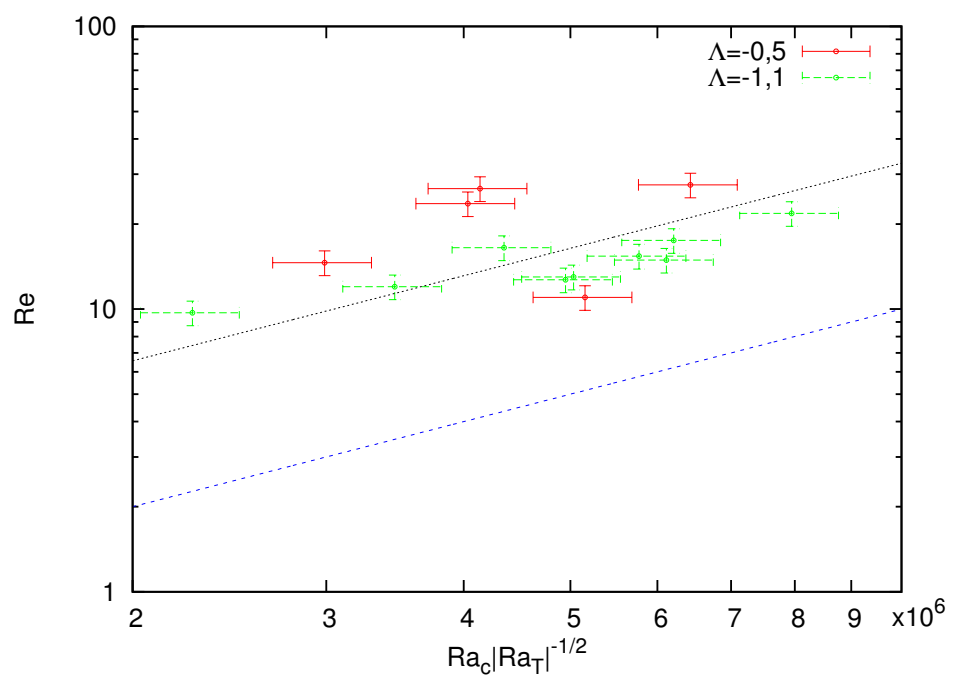

ABB. 4.10: Überprüfung der Gleichung 3.5 für die Finger in der thermohalinen Treppe unter der Annahme für einen Konzentrationsgradienten von $\Delta c /(2 L)$ und einen Temperaturgradienten von $\Delta T /(2 L)$. Die blaue Linie ist für den Vorfaktor $10^{-6}$, die schwarze für den Vorfaktor $3,3 \cdot 10^{-6}$. Die Abweichung kommt durch die unterschiedlichen Randbedingungen.

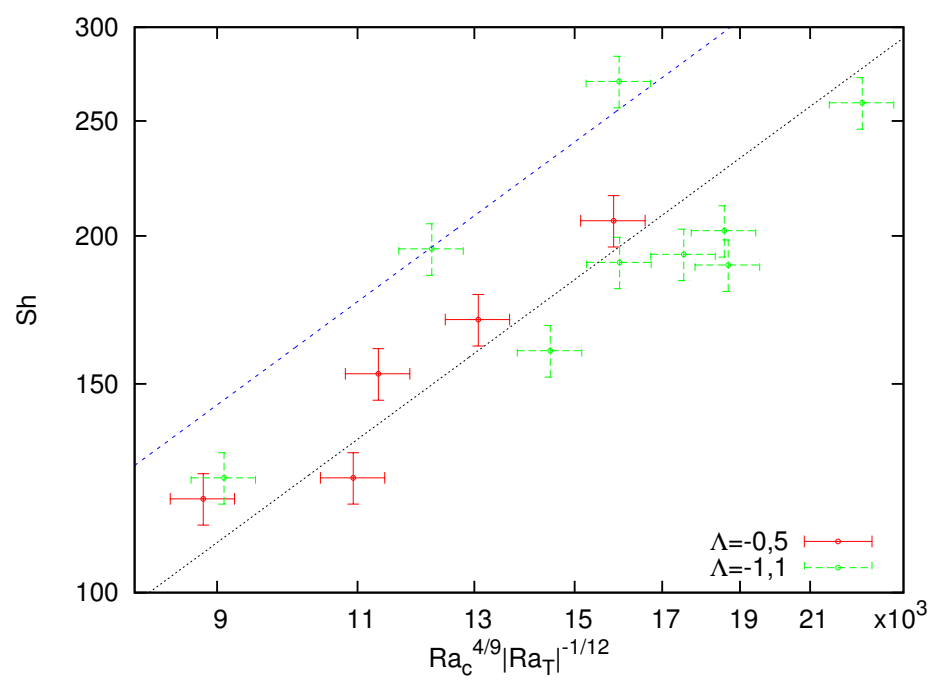

ABB. 4.11: Überprüfung der Gleichung 3.6 für die Finger in der thermohalinen Treppe unter der Annahme für einen Konzentrationsgradienten von $\Delta c /(2 L)$ und einen Temperaturgradienten von $\Delta T /(2 L)$. Die blaue Linie ist für den Vorfaktor 0,016, die schwarze für den Vorfaktor 0,0122. Die Abweichung kommt durch die unterschiedlichen Randbedingungen. 


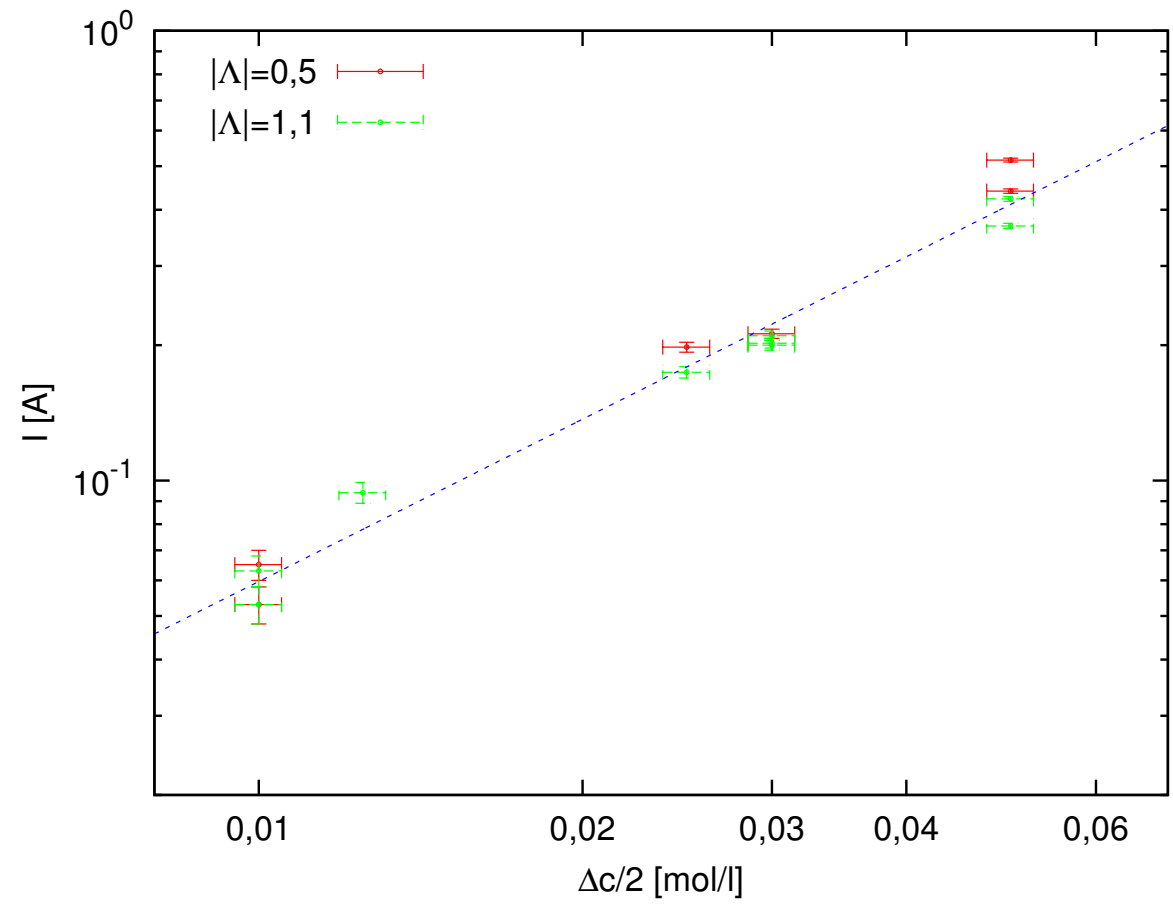

AвB. 4.12: $I \sim f(\Lambda, L e, \operatorname{Pr})(\Delta c / 2)^{x}$ über ein Fingerinterface für die beiden Auftriebsverhältnisse aufgetragen. Die blaue Linie ist der beste Fit aller Datenpunkte mit $I=6,69 \cdot(\Delta c / 2)^{1,21}$. 



\section{Zusammenfassung}

Im ersten Teil dieser Arbeit wurde mit Hilfe einer elektrochemischen Zelle das Verhalten von doppelt diffusiver Konvektion im Fingerregime untersucht. Die Zelle hatte einen quadratischen Grundriss von ca. 50 Fingerdicken $(20 \mathrm{~cm})$ und eine variable Höhe mit Aspektverhältnissen von $\Gamma=1,5 / 2,10 / 3,5,20 / 3$ und 10. Die schneller diffundierende Komponente war Wärme $\left(\kappa_{T}=1,24 \cdot 10^{-7}\right)$ und die langsamer diffundierende Komponente waren Kupferionen $\left(\kappa_{c}=5,51 \cdot 10^{-10}\right)$. Die Prandtlzahlen und Schmidtzahlen waren annähernd konstant mit $8,55<\operatorname{Pr}<8,99$ und $1910<S c<2114$. Dies entspricht einer Lewiszahl von ca. 227. Die thermische Rayleighzahl wurde von $\approx 10^{4}$ bis $\approx 10^{9}$ und die chemische Rayleighzahl von $\approx 10^{9}$ bis $\approx 2 \cdot 10^{12}$ variiert. Alle Messungen wurden im Bereich $0,02<|\Lambda|<0,37$ gemacht. In diesem Bereich ist die Dichte instabil geschichtet und man nahm bis vor Kurzem an, dass dort keine Finger entstehen könnten. Durch die Ergebnisse von [Hage and Tilgner, 2010] und [Yang et al., 2015b] weiß man nun, dass Finger auch im instabil geschichteten Medium existieren können. Der Übergang von Rayleigh-Bénard-Konvektion zu Fingerkonvektion liegt bei einem Auftriebsverhältnis $|\Lambda|=\frac{\left|\alpha_{T} \Delta T\right|}{\alpha_{c} \Delta c}=1 / 30$ und ist eine überkritische Bifurkation. Der instabile Einfluss der langsamer diffundieren Komponente am Übergang ist dreißig mal größer als der stabilisierende der schneller diffundieren Komponente. Diese Grenze hängt allerdings auch von der Lewiszahl ab und steigt mit sinkender Lewiszahl. Für $L e=10$ erwartet man z.B. einen Übergang bei $|\Lambda|=1 / 6$ (vgl. [Müller, 2013, Yang et al., 2015a]). Dass die Grenze bei $|\Lambda|=1 / 30$ liegt, bedeutet nach Gleichung 2.12, dass der Bereich der Fingerströmung auf $45^{\circ}<T u<133,1^{\circ}$ für $L e=227$ erweitert werden muss.

Eine Ursache, warum Finger im instabil geschichteten Medium bis zu einem Auftriebsverhältnis von $-1 / 30$ existieren können, konnte bis jetzt und auch durch diese Arbeit nicht gefunden werden. Die Fingerreynoldszahl $R e_{f}$ ist zu klein, um die Finger zu destabilisieren, die chemische Grenzschichtrayleighzahl $R a_{c, \lambda}$ ist unterkritisch, genau wie die Grenzschichtrayleighzahl $R a_{\lambda}$. Das Sternkriterium kann genauso verworfen werden wie die Instabilitäten der Kolmogorov-Strömung. Der Gradient des Temperaturfeldes am Übergang ist nicht verschwunden. Selbst die Temperaturdiffusion, die in dieser Arbeit 


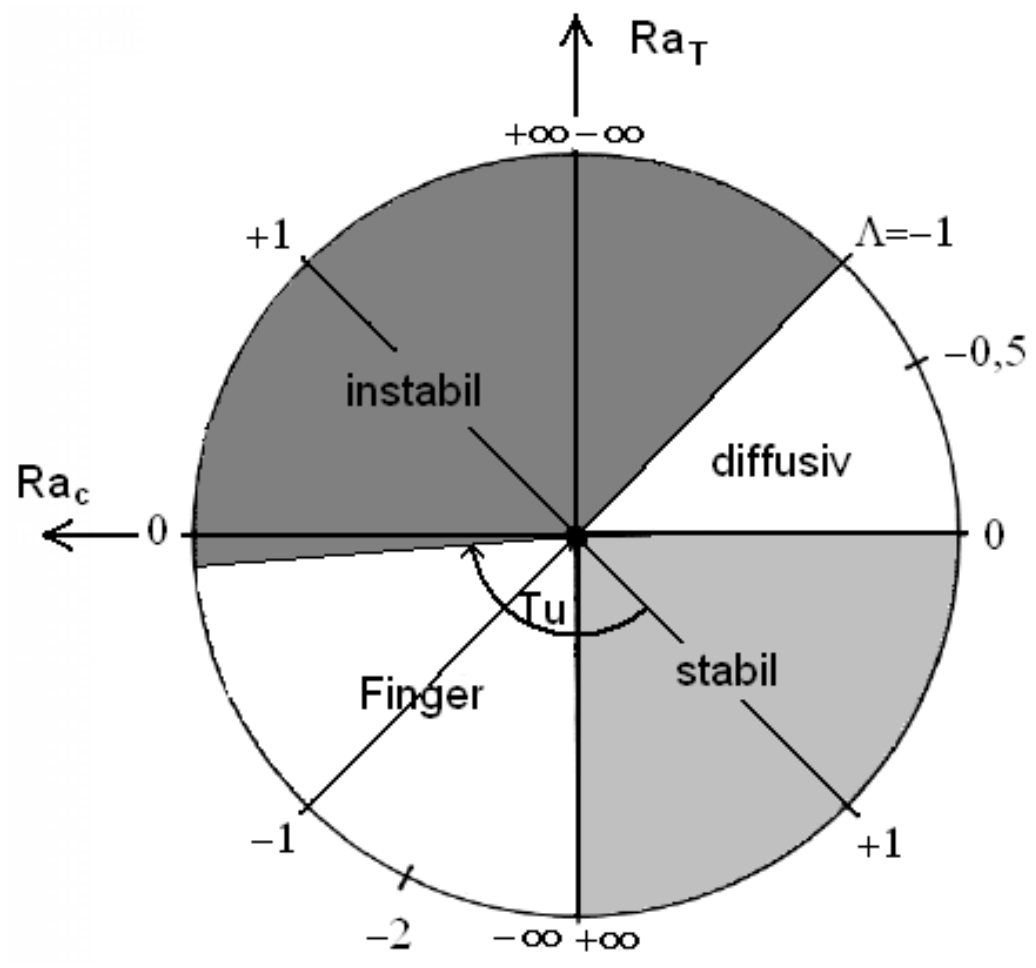

ABB. 5.1: Schematische Darstellung des doppelt diffusiven Regimes über den Turnerwinkel (Vgl. [Ruddick, 1983]) mit der Erweiterung des Fingerregimes auf $45^{\circ}<T u<133,1^{\circ}$.

der aussichtsreichste Kandidat ist, sorgt höchstwahrscheinlich bei kleinen Le nicht dafür, dass die Finger instabil werden (vgl. [Yang et al., 2015a]).

Eine ähnliche Frage stellt sich auch bei Rayleigh-Bénard-Konvektion. Warum bilden sich Konvektionsrollen bei hohen Rayleighzahlen? Der Dichteausgleich könnte genauso durch individuelle Plumes entstehen, die die Zelle wie Blasen in einen Kochtopf durchqueren. Natürlich ist klar, das Konvektionswalzen bei hohen Rayleighzahlen entstehen, aber nur eine kleine Störung von -1/30 im Auftriebsverhältnis sorgt dafür, dass sie durch kleinskalige Strukturen ersetzt werden.

Die Gesetzmäßigkeiten von [Hage and Tilgner, 2010] und das 4/3-Gesetz verlieren teilweise ihre Gültigkeit nahe des Überganges. Die Fingerdicke verhält sich noch im Rahmen der Fehlertoleranz wie vorhergesagt. Für die Sherwoodzahl und die Reynoldszahl müssen die Potenzgesetze angepasst werden.

Zuletzt sei noch erwähnt, dass die Rolle der Ränder in dieser Arbeit nicht genau bestimmt werden kann. In dieser Arbeit entsteht Konvektion durch Rayleigh-Taylor-Instabilitäten 
der chemischen Grenzschicht. Diese wachsen entweder zu Fingern, wenn der Temperaturgradient groß genug ist, oder zu Konvektionswalzen, wenn er zu klein ist. Im Ozean z.B. existieren solche Grenzen nicht. Finger tauchen dort oft in thermohalinen Treppen auf, die keine festen Ränder, sondern Konvektionsbereiche haben. Daher muss erst noch überprüft werden, ob Finger unter solchen Bedingungen auch für Auftriebsverhältnisse bis hinunter zu 1/30 existieren können.

Im zweiten Teil der Arbeit wurden thermohaline Treppen im Fingerregime untersucht. Dabei wurde die Zelle aus dem ersten Teil angepasst. Der quadratische Grundriss blieb erhalten, wurde aber auf $10 \mathrm{~cm}(25 d)$ Kantenlänge verkleinert. Die Höhe der Zelle war wieder variabel mit Aspektverhältnissen von $\Gamma=1 / 4$ und 1/8. Durch eine neue Befülltechnik wurde ein stabiler Temperaturgradient direkt beim Befüllen erzeugt. Die Prandtlund Schmidtzahlen variierten von 7,42<Pr $<8,38$ und $1407<S c<1835^{1}$. Die thermische Rayleighzahl wurde $\approx 6 \cdot 10^{9}$ bis $\approx 6 \cdot 10^{11}$ und die chemische Rayleighzahl von $\approx 2 \cdot 10^{12}$ bis $\approx 10^{14}$ variiert. Dabei wurde das Auftriebsverhältnis jeweils konstant auf $\Lambda=-0,5$ oder $\Lambda=-1,1$ gehalten, sodass die thermohalinen Treppen im klassischen Fingerregime mit denen im neuen Fingerregime verglichen werden konnten.

Es bildeten sich thermohaline Treppen mit zwei Fingerbereichen und einem Konvektionsbereich dazwischen. Die Theorien zur Entstehung von thermohalinen Treppen konnten nicht überprüft werden, vor allem, da der Wärmefluss nicht gemessen werden konnte und somit das Verhalten von $\gamma$ als Funktion von $\Lambda$ unbekannt ist. Auch war es nicht möglich wie z.B. bei [Krishnamurti, 2003] lokal die Konzentration und die Temperatur zu messen. Eine Annahme von $\Delta c /(2 L)$ als Konzentrationsgradienten und $\Delta T /(2 L)$ als Temperaturgradienten scheint plausibel, muss aber noch bestätigt werden. Sollten diese Gradienten vorliegen, so verhalten sich die Finger nach den Gleichungen 3.4, 3.5 und 3.6 aus [Hage and Tilgner, 2010] und der Massenfluss mit kleinen Einschränkungen nach dem 4/3-Gesetz von [Turner, 1965, Turner, 1967].

Zusammengefasst lässt sich sagen, dass keine bemerkbaren Unterschiede zwischen den thermohalinen Treppen bei instabilem Auftriebsverhältnis zu den thermohalinen Treppen bei stabilem Auftriebsverhältnis gefunden wurden. Trotz allem bleiben noch einige offene Fragen:

\footnotetext{
${ }^{1}$ Daraus ergibt sich eine Variation von $189<L e<219$ und ist somit nur geringfügig größer als für den ersten Teil.
} 
- Was ist der Konzentrations- und der Temperaturgradient über der Fingerzone?

- Wie sieht der Übergang zwischen Finger und Konvektion im Strömungsbild aus (Randbedingungen für die Finger)?

- Wie verhalten sich die Treppen bei noch instabileren Auftriebsverhältnissen, bis Nahe des Übergang zum konvektiven Regime?

- Wieso ist die Geschwindigkeitsrandschicht von $v_{y}$ so groß und warum scheinen die Finger bei den beiden horizontalen Raumrichtungen eine Vorzugsrichtung zu haben?

- Und vor allem, wie entstehen diese Treppen?

Einige dieser Fragen lassen sich relativ schnell durch weitere Messungen beantworten. Das Temperaturprofil könnte man mit Thermokristallen sichtbar machen und somit $\Delta T$ bestimmen. Das minimal erreichbare Auftriebsverhältnis beträgt $|\Lambda|=0,01$, sodass man weitere Messungen bei Auftriebsverhältnissen nahe des Überganges zum konvektiven Regime mit diesem Messaufbau durchführen kann. Um von 2D zu 3D- Aufnahmen zu wechseln, oder den Temperaturfluss zu bestimmen kann man das Experiment nicht ohne weiteres erweitern.

Zuletzt sieht man in Abbildung 5.2 alle in dieser Arbeit aufgenommenen Messpunkte im $R a_{c}-R a_{T}$-Phasenraum. Zur Übersicht sind die Messungen von [Hage, 2009] mit eingetragen. Die rote gestrichelte Linie stellt $|\Lambda|=1$ dar. Der Übergang vom Fingerregime zum Konvektionsregime ist durch die schwarze Linie mit $|\Lambda|=1 / 30$ dargestellt. Die durchgezogene Linie markiert die Relation 4.3. Oberhalb derer sollten keine Treppen zu finden sein. Man erkennt aber auch, dass nicht nur die Fingerlänge ein Kriterium darstellt, da sonst in der Messreihe von Kapitel 3 und [Hage, 2009] thermohaline Treppen hätten gefunden werden müssen. Es bedarf weiterer Arbeit, dieses zusätzliche Kriterium zu finden. Vor allem ist es notwendig herauszufinden, was dafür sorgt, dass thermohaline Treppen entstehen. 


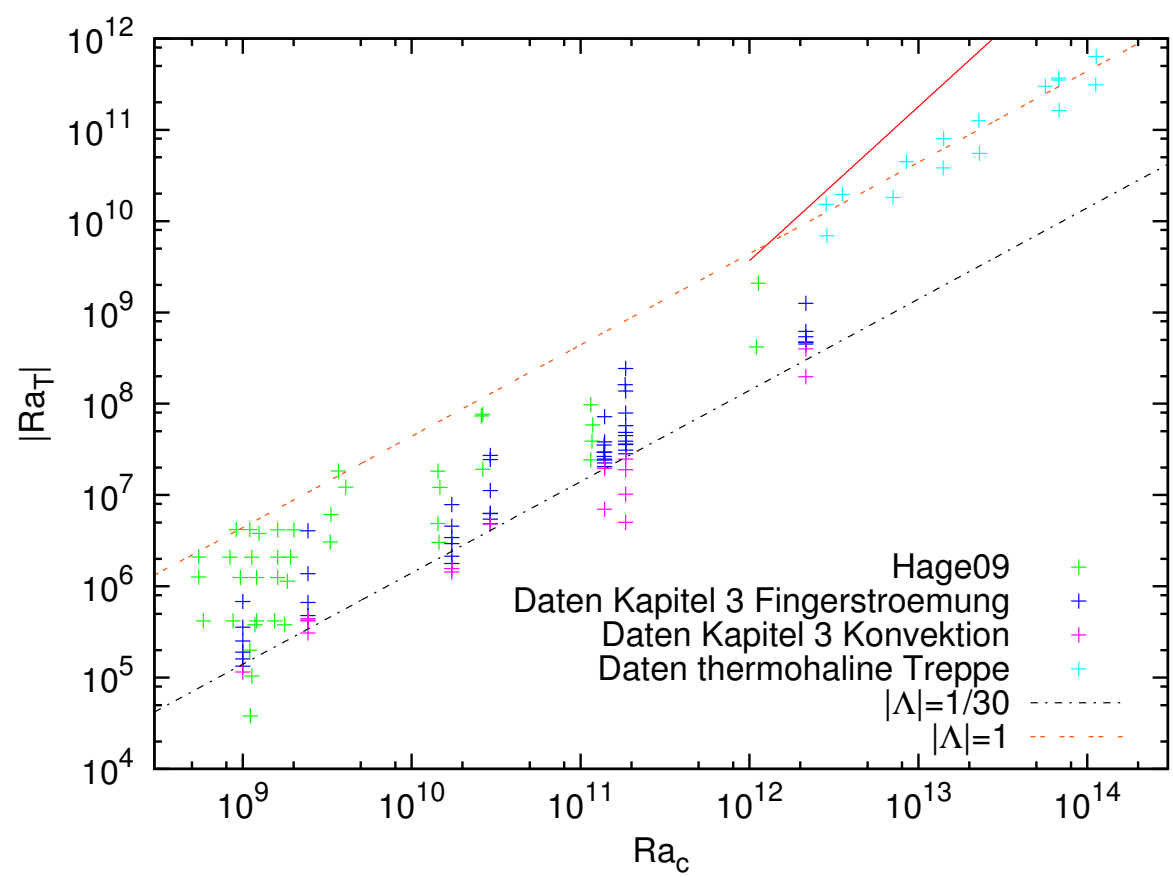

ABB. 5.2: $R a_{c}-R a_{T}$-Phasenraum mit allen Messdaten dieser Arbeit im Fingerregime und den Messdaten von [Hage, 2009]. Die gestrichelte rote Linie stellt das neutrale Auftriebsverhältnis dar. Die schwarze Linie markiert den Übergang von Konvektion zu Fingerströmung. Die durchgezogene rote Linie ist die Relation 4.3 oberhalb derer keine thermohalinen Treppen entstehen sollten. 



\section{Nomenklatur}

Die wichtigsten in dieser Arbeit verwendeten Größen sind hier noch einmal zusammengefasst.

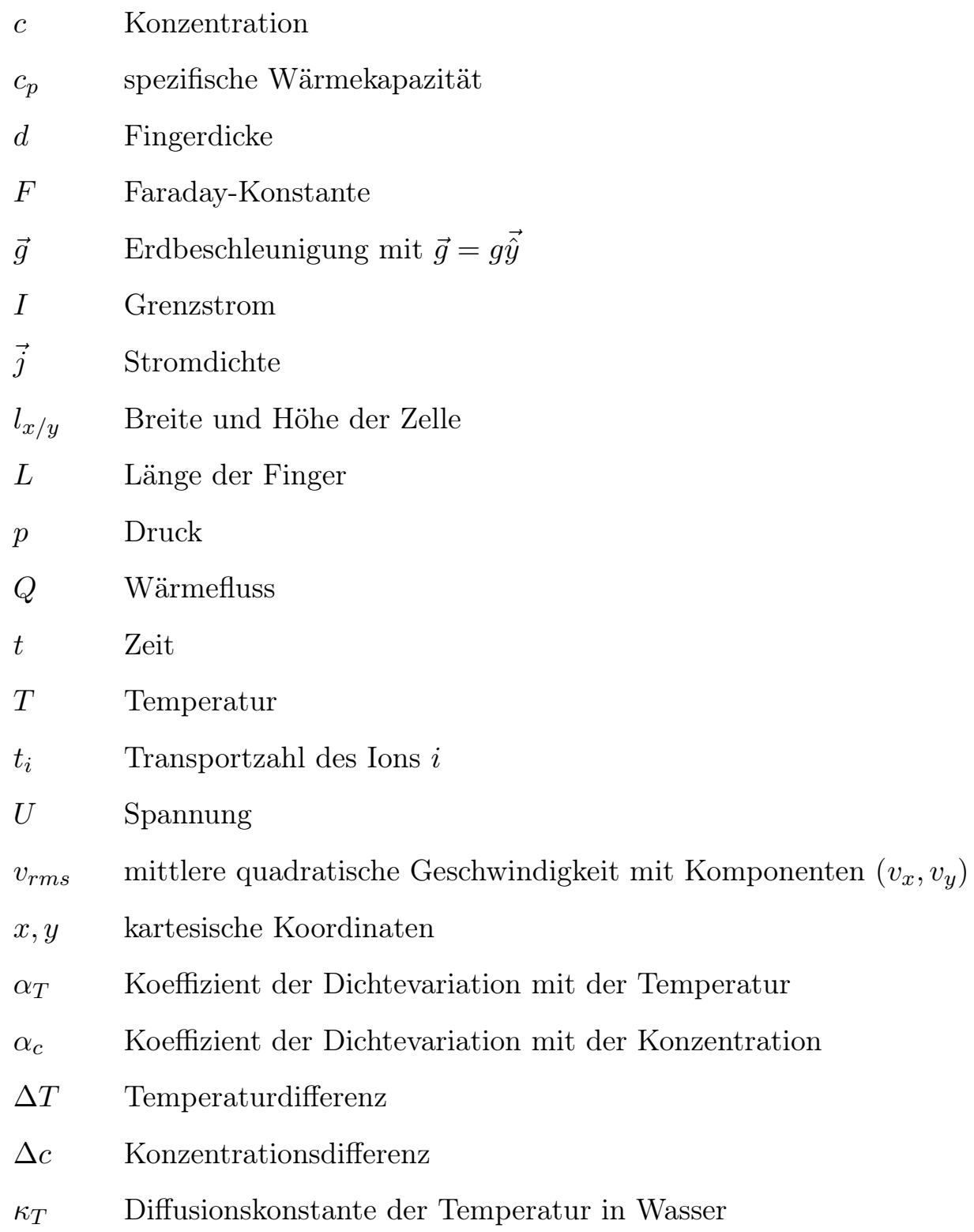


$\kappa_{c} \quad$ Diffusionskonstante des Stoffes in Wasser

$\lambda_{c}, \lambda_{T}$ chemische und thermische Grenzschicht

$\mu \quad$ dynamische Viskosität

$\nu \quad$ kinematische Viskosität

$\rho \quad$ Dichte

$\phi \quad$ elektrisches Potential

\section{Dimensionslose Kennzahlen}

$R a_{T} \quad$ thermische Rayleighzahl $\frac{g \alpha_{T} \Delta T l_{y}^{3}}{\kappa_{T} \nu}$

$R a_{c} \quad$ chemische Rayleighzahl $\frac{g \alpha_{c} \Delta c l_{y}^{3}}{\kappa_{c} \nu}$

$R a_{\lambda} \quad$ Grenzschichtrayleighzahl $R a_{\lambda}=\frac{g \lambda_{c}^{4}}{\nu}\left(\frac{\Delta c}{\kappa_{c} l_{y}}-\frac{\Delta T}{\kappa_{T} l_{y}}\right)$

$\operatorname{Pr} \quad$ Prandtlzahl $\quad \nu / \kappa_{T}$

Sc Schmidtzahl $\quad \nu / \kappa_{c}$

Le Lewiszahl $\kappa_{T} / \kappa_{c}$

Sh Sherwoodzahl $\quad \frac{j l_{y}}{z F \kappa_{c} \Delta c}$

$R e_{x / y} \quad$ Reynoldszahl in $\mathrm{x} / \mathrm{y} \quad \frac{v_{x / y} l_{y}}{\nu}$

Re Reynoldszahl $\quad \frac{v_{r m s} l_{y}}{\nu}=\sqrt{R e_{x}^{2}+R e_{y}^{2}}$

$R e_{f} \quad$ Fingerreynoldszahl $\quad R e \frac{d}{l_{y}}=\frac{v_{r m s} d}{\nu}$

$\Gamma \quad$ Aspektverhältnis $\quad l_{x} / l_{y}$

$\gamma \quad$ Flussverhältnis $\quad \frac{\alpha_{T} Q}{\alpha_{c} j}$

$\Lambda \quad$ Auftriebsverhältnis $\quad \frac{\alpha_{T} \Delta T}{\alpha_{c} \Delta c}=L e \frac{R a_{T}}{R a_{c}}$ 


\section{Apendix}

\subsection{Abbildungen und Messdaten}
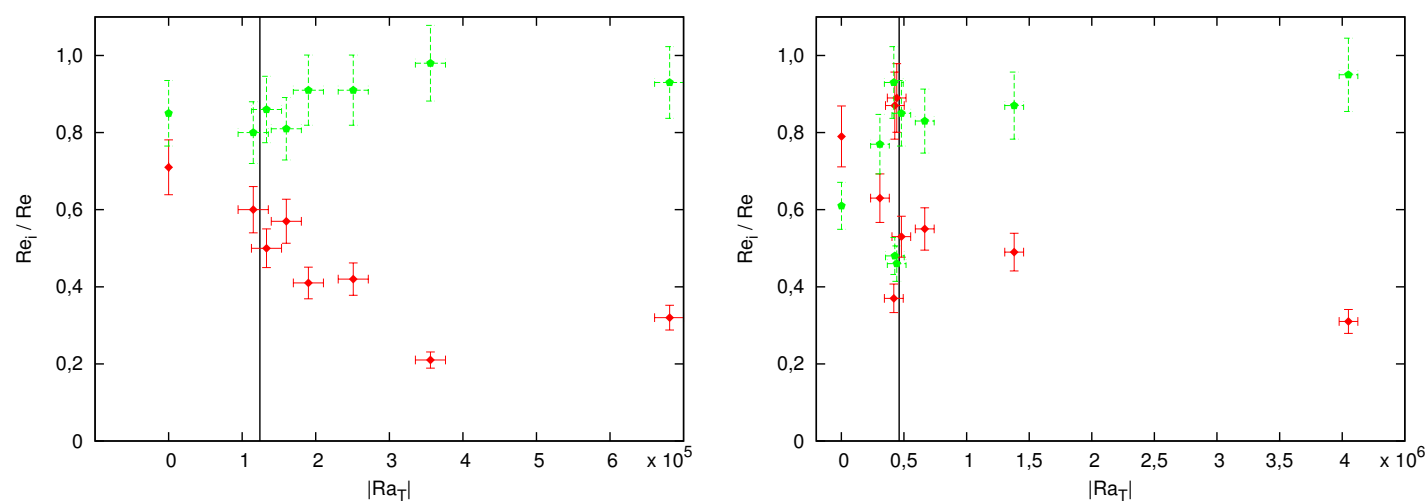

ABB. 7.1: $R e_{x} / R e$ (rot) und $R e_{y} / R e$ (grün) als Funktion von $\left|R a_{T}\right|$ bei $R a_{c}=1 \cdot 10^{9}$ (links) und $R a_{c}=2,43 \cdot 10^{9}$ (rechts) aufgetragen.
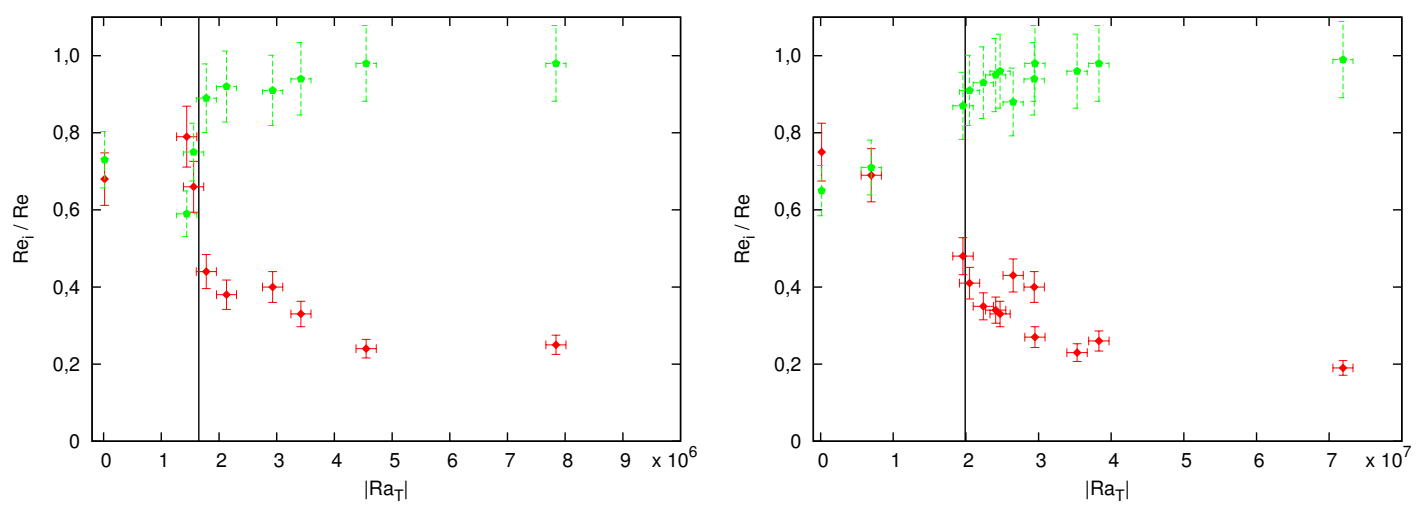

ABB. 7.2: $R e_{x} / R e$ (rot) und $R e_{y} / R e$ (grün) als Funktion von $\left|R a_{T}\right|$ bei $R a_{c}=1,73 \cdot 10^{10}$ (links) und $R a_{c}=1,39 \cdot 10^{11}$ (rechts) aufgetragen. 

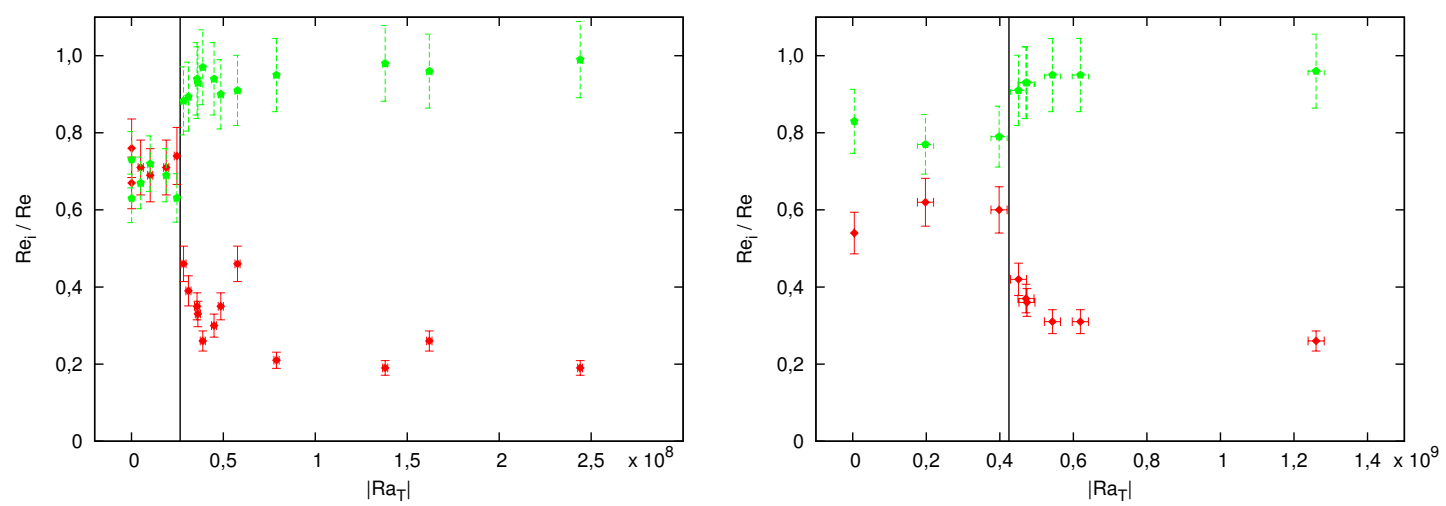

AвB. 7.3: $R e_{x} / R e\left(\right.$ rot) und $R e_{y} / R e$ (grün) als Funktion von $\left|R a_{T}\right|$ bei $R a_{c}=1,85 \cdot 10^{11}$ (links) und $R a_{c}=2,16 \cdot 10^{12}$ (rechts) aufgetragen.
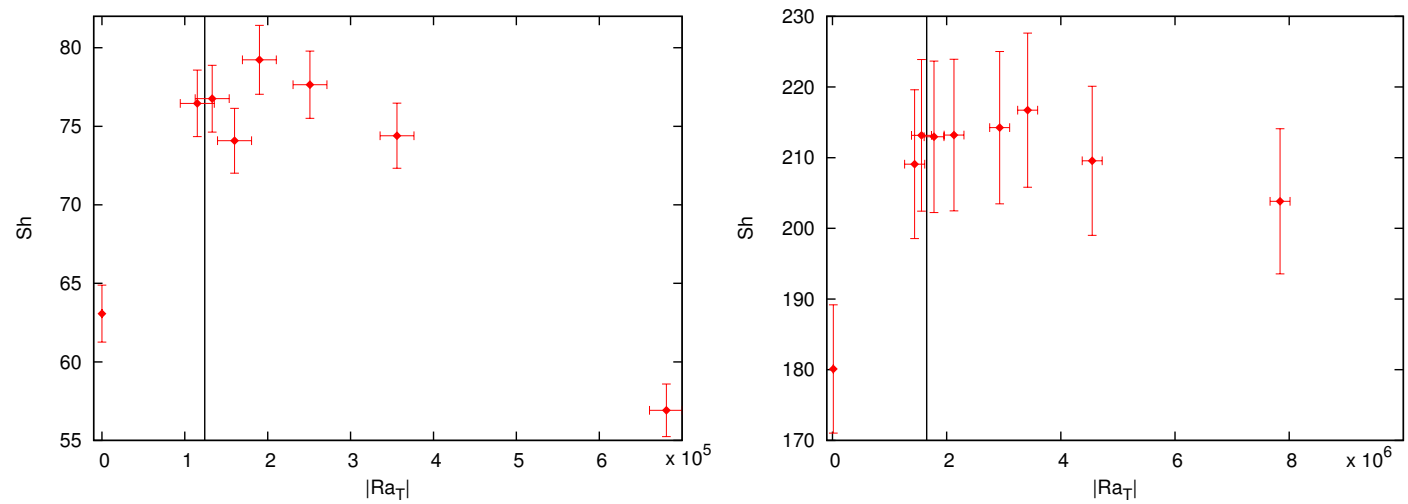

ABB. 7.4: $S h$ als Funktion von $\left|R a_{T}\right|$ bzw. $|\Lambda|$ für $R a_{c}=1 \cdot 10^{9}$ (links) und $R a_{c}=$ $1,73 \cdot 10^{10}$ (rechts) aufgetragen.
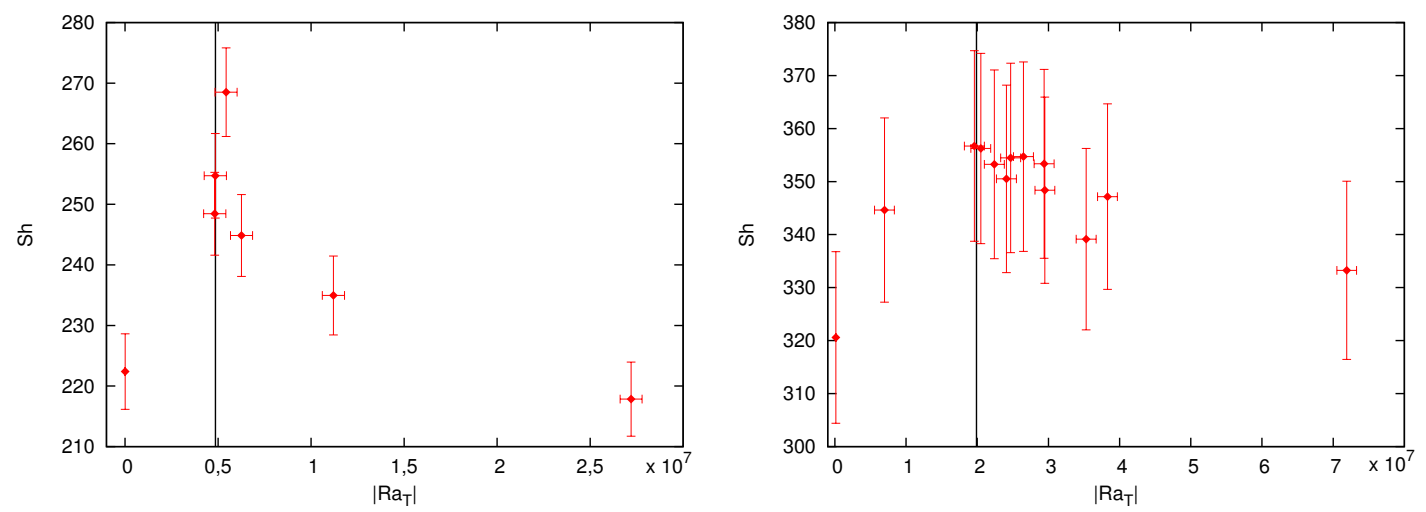

ABB. 7.5: $S h$ als Funktion von $\left|R a_{T}\right|$ bzw. $|\Lambda|$ für $R a_{c}=2,92 \cdot 10^{10}$ (links) und $R a_{c}=$ $1,39 \cdot 10^{11}$ (rechts) aufgetragen. 

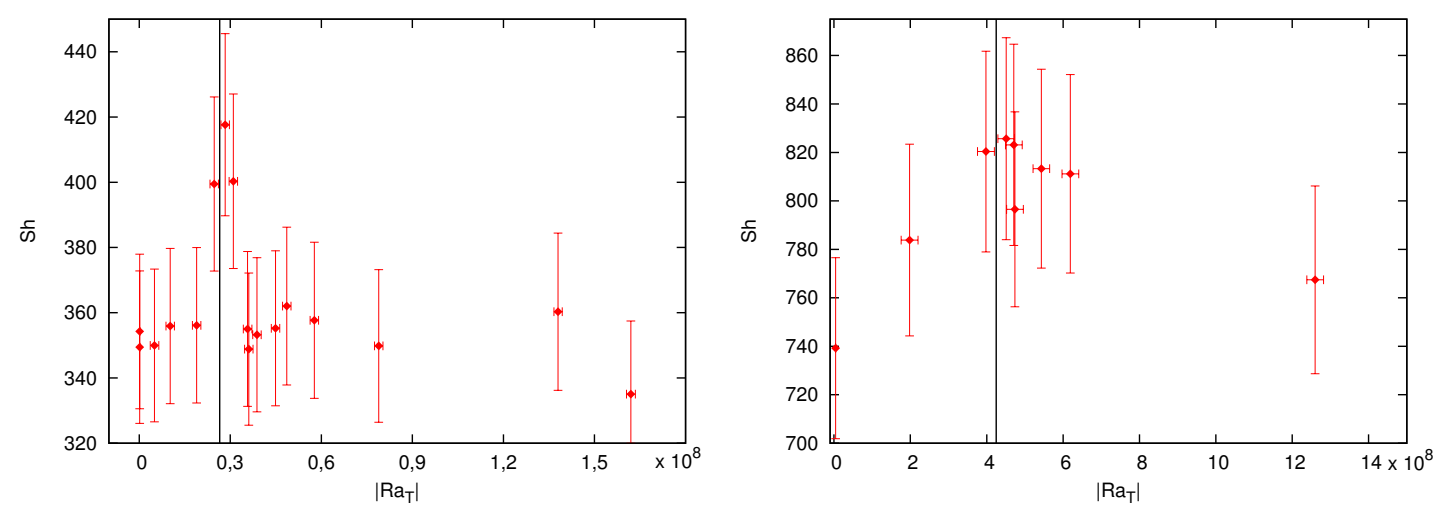

AвB. 7.6: $S h$ als Funktion von $\left|R a_{T}\right|$ bzw. $|\Lambda|$ für $R a_{c}=1,85 \cdot 10^{11}$ (links) und $R a_{c}=$ $2,16 \cdot 10^{12}$ (rechts) aufgetragen.
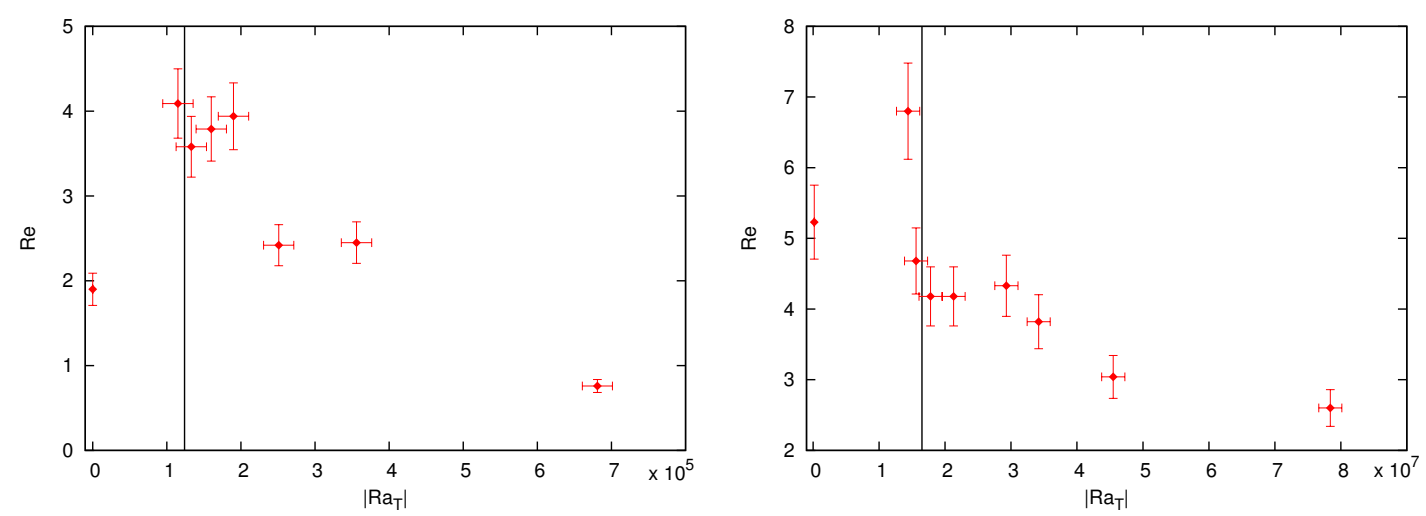

AвB. 7.7: $R e$ als Funktion von $\left|R a_{T}\right|$ bzw. $|\Lambda|$ für $R a_{c}=1 \cdot 10^{9}$ (links) und $R a_{c}=$ $1,73 \cdot 10^{10}$ (rechts) aufgetragen.
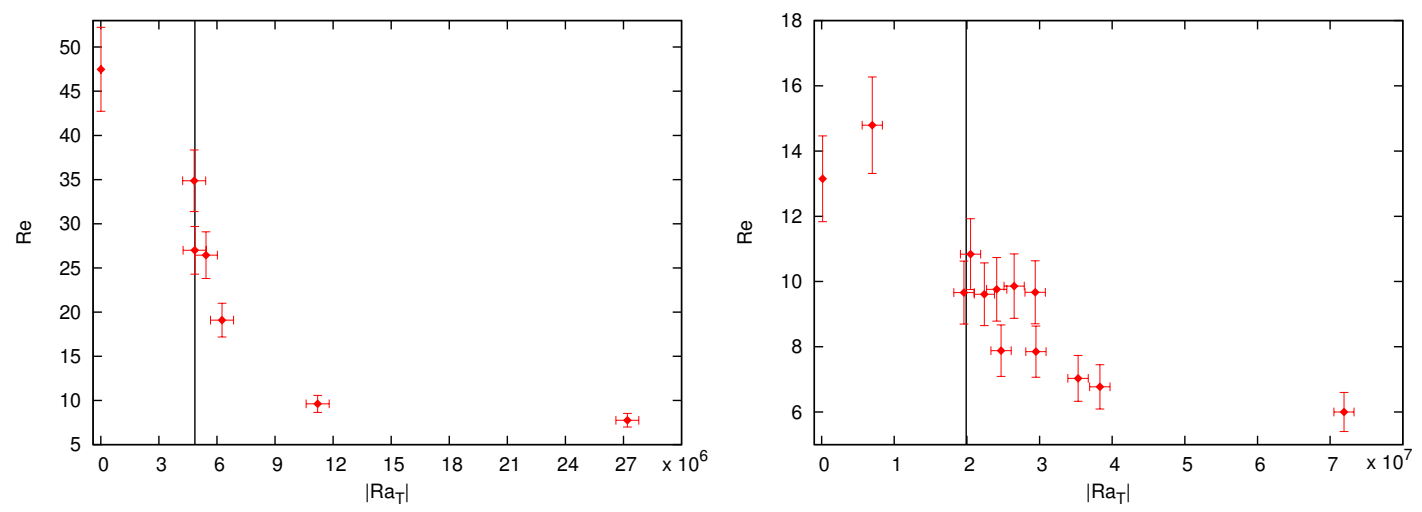

ABB. 7.8: $R e$ als Funktion von $\left|R a_{T}\right|$ bzw. $|\Lambda|$ für $R a_{c}=2,92 \cdot 10^{10}$ (links) und $R a_{c}=$ $1,39 \cdot 10^{11}$ (rechts) aufgetragen. 

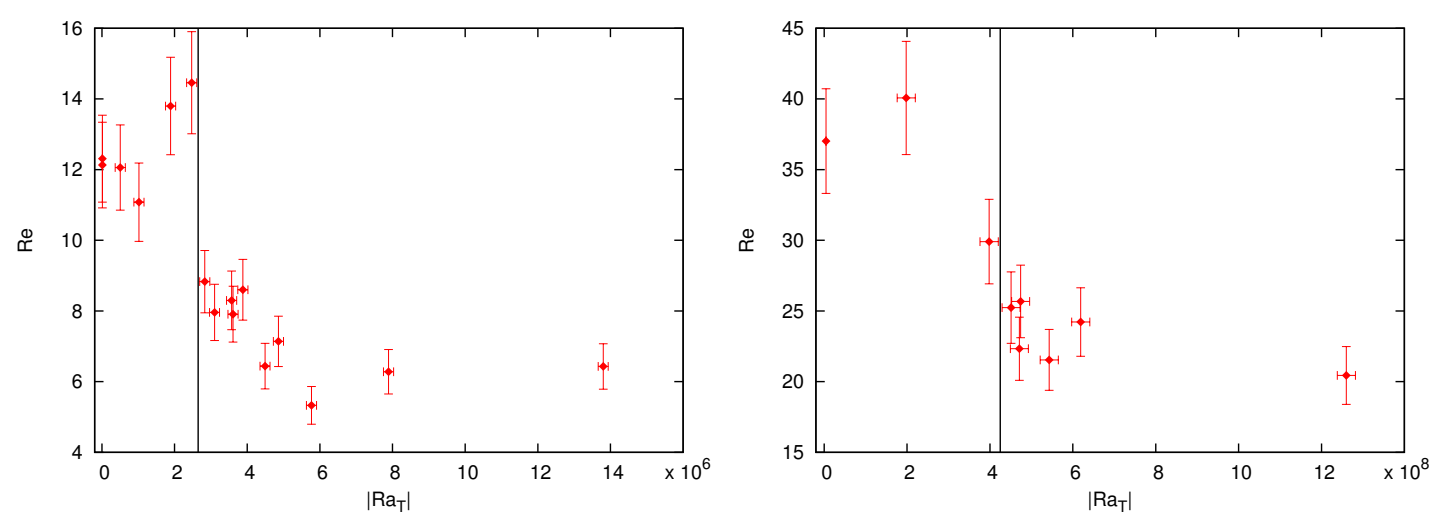

ABв. 7.9: $R e$ als Funktion von $\left|R a_{T}\right|$ bzw. $|\Lambda|$ für $R a_{c}=1,85 \cdot 10^{11}$ (links) und $R a_{c}=$ $2,16 \cdot 10^{12}$ (rechts) aufgetragen.

\subsection{Ergebnistabellen}

Alle Messdaten sind hier in tabellarischer Form hinterlegt.

Tabelle 7.1 bis 7.7 beinhalten die Messdaten für die Bestimmung des Überganges vom Fingerregime zum konvektiven Regime (siehe Kapitel 3.3). Die Daten sind absteigend nach $\left|R a_{T}\right|$ sortiert.

Tabelle 7.8 und 7.9 beinhalten die Daten für die Messungen zu den thermohalinen Treppen. Die Daten sind nach aufsteigenden $R a_{c}$ sortiert. 


\begin{tabular}{|c|c|c|c|c|c|c|c|c|}
\hline$\tilde{\omega}$ & in & I & $\infty$ & $\mathbb{R}$ & $\underset{N}{\mathbb{N}}$ & N & N & 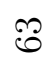 \\
\hline$<$ & $\begin{array}{l}\stackrel{H}{20} \\
\stackrel{2}{0}\end{array}$ & $\begin{array}{l}0 \\
0 \\
0 \\
0 \\
0\end{array}$ & $\begin{array}{l}1 \\
\stackrel{1}{0} \\
0 \\
0\end{array}$ & 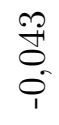 & 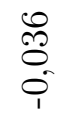 & $\begin{array}{l}0 \\
\text { Oి } \\
0 \\
0\end{array}$ & $\begin{array}{l}0 \\
\stackrel{N}{0} \\
0 \\
0\end{array}$ & $\begin{array}{l}8 \\
8 \\
0 \\
0\end{array}$ \\
\hline$\frac{\bar{\Xi}}{-1}$ & $\underset{\perp}{F}$ & 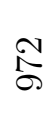 & $\underset{\overrightarrow{0}}{\overrightarrow{0}}$ & $\begin{array}{l}0 \\
\stackrel{0}{0}\end{array}$ & $\mathscr{\mathscr { \theta }}$ & $\underset{8}{8}$ & $\underset{8}{8}$ & $\underset{\infty}{\mathbb{N}}$ \\
\hline$\frac{\hat{y}^{2}}{{ }_{2}^{2}}$ & ले & $\vec{m}$ & $\stackrel{\stackrel{ి}{I}}{\sim}$ & $\stackrel{\mathscr{L}}{-}$ & 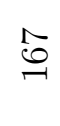 & $\underset{-1}{0}$ & $\stackrel{\infty}{\stackrel{\infty}{\sim}}$ & $\infty$ \\
\hline$\frac{\hat{y}^{\infty} \infty}{\overrightarrow{2}^{\infty}}$ & $\stackrel{m}{\longrightarrow}$ & $\stackrel{\infty}{\sim}$ & $\begin{array}{l}10 \\
10\end{array}$ & $\stackrel{\infty}{\infty}$ & $\stackrel{\infty}{=}$ & $\stackrel{\infty}{\infty}$ & $\stackrel{m}{9}$ & $\stackrel{+}{N}$ \\
\hline $\begin{array}{c}\text { है। } \\
\text { हैं } \\
\text { है }\end{array}$ & $\stackrel{\text { Fै }}{ }$ & 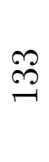 & 囚ิ & $\stackrel{10}{\sim}$ & $\stackrel{\sim}{\circ}$ & $\stackrel{\mathscr{L}}{\Omega}$ & $\stackrel{\Re}{\stackrel{N}{N}}$ & $\underset{ }{\stackrel{-}{\circ}}$ \\
\hline$\frac{\bar{\Xi}}{\bar{\sigma}}$ & $\begin{array}{l}\mathcal{O} \\
\text { ô }\end{array}$ & $\begin{array}{l}\text { aे } \\
\text { is }\end{array}$ & $\begin{array}{l}\infty \\
\infty \\
\infty\end{array}$ & So & $\begin{array}{l}\stackrel{0}{1} \\
\infty\end{array}$ & $\begin{array}{l}18 \\
0 \\
0\end{array}$ & 1 & 1 \\
\hline$\AA^{2}$ & $\begin{array}{l}\overrightarrow{0} \\
\infty\end{array}$ & \begin{tabular}{c}
\multirow{0}{0}{} \\
$\infty$
\end{tabular} & $\begin{array}{l}\tilde{b} \\
\infty\end{array}$ & \begin{tabular}{c}
\multirow{0}{0}{} \\
$\infty$
\end{tabular} & $\overrightarrow{0}$ & $\begin{array}{l}\stackrel{8}{0} \\
\infty\end{array}$ & $\overrightarrow{6}$. & $\mathscr{b}_{\infty}^{0}$ \\
\hline जs & $\stackrel{\overrightarrow{2}}{\stackrel{2}{\Omega}}$ & $\underset{\sigma}{\vec{\sigma}}$ & $\stackrel{\mathscr{P}}{\stackrel{\leftrightarrow}{\sim}}$ & 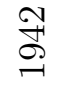 & $\stackrel{\sim}{\mathscr{\sigma}}$ & 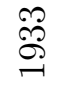 & $\begin{array}{l}\infty \\
\stackrel{\infty}{=} \\
\stackrel{-}{-1}\end{array}$ & $\begin{array}{l}\stackrel{\circ}{\mathscr{D}} \\
\stackrel{2}{二}\end{array}$ \\
\hline ב) & $\begin{array}{l}\stackrel{8}{\rho} \\
\stackrel{1}{-1} \\
\infty \\
0 \\
1\end{array}$ & $\begin{array}{l}18 \\
\stackrel{0}{1} \\
\dot{0} \\
10 \\
0 \\
1\end{array}$ & $\begin{array}{l}18 \\
0 \\
\dot{1} \\
10 \\
\text { of } \\
1\end{array}$ & $\begin{array}{l}\stackrel{8}{\circ} \\
\dot{0} \\
\dot{8} \\
i \\
1\end{array}$ & $\begin{array}{l}18 \\
0 \\
\dot{0} \\
0 \\
1\end{array}$ & 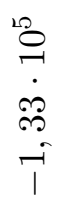 & 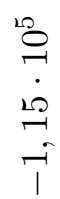 & $\begin{array}{l}8 \\
0 \\
\dot{8} \\
\dot{8} \\
\dot{0}\end{array}$ \\
\hline$\frac{\bar{E}}{E}$ & $\overbrace{}^{20}$ & $\stackrel{+}{F}$ & $\stackrel{2}{\stackrel{2}{7}}$ & $\begin{array}{l}\infty \\
0 \\
0 \\
0\end{array}$ & $\begin{array}{l}\infty \\
1 \\
0 \\
0\end{array}$ & $\begin{array}{l}10 \\
0 \\
0 \\
1\end{array}$ & $\begin{array}{l}0 \\
10 \\
0 \\
0\end{array}$ & $\begin{array}{l}8 \\
0 \\
i\end{array}$ \\
\hline $\begin{array}{l}\text { ㅎํㄴ } \\
\dot{y}\end{array}$ & $\begin{array}{l}\mathscr{8} \\
0 \\
0\end{array}$ & $\begin{array}{l}8 \\
0\end{array}$ & $\begin{array}{l}8 \\
0 \\
0\end{array}$ & $\begin{array}{l}8 \\
0 \\
0\end{array}$ & $\begin{array}{l}\mathscr{0} \\
0\end{array}$ & $\begin{array}{l}8 \\
0 \\
0\end{array}$ & $\begin{array}{l}8 \\
0 \\
0\end{array}$ & $\begin{array}{l}8 \\
0 \\
0\end{array}$ \\
\hline 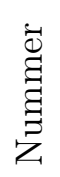 & 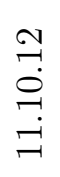 & 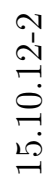 & $\begin{array}{l}\stackrel{N}{?} \\
\stackrel{0}{-} \\
\stackrel{-}{0}\end{array}$ & 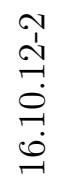 & $\begin{array}{l}\stackrel{N}{\leftrightarrows} \\
\stackrel{0}{+} \\
\stackrel{\infty}{=}\end{array}$ & 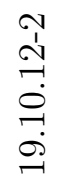 & 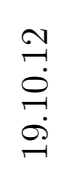 & 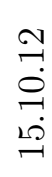 \\
\hline
\end{tabular}

要

\begin{tabular}{|c|c|c|c|c|c|c|}
\hline $\bar{m}$ & $\underset{-}{\stackrel{1}{0}}$ & $\stackrel{\overbrace{}}{\stackrel{ }{\sim}}$ & $\overrightarrow{0}$ & $\stackrel{\text { mo }}{\sim}$ & $\stackrel{\vec{N}}{\sim}$ & $\stackrel{1}{-1}$ \\
\hline$<$ & 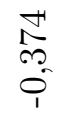 & $\begin{array}{l}\text { N } \\
\stackrel{N}{O} \\
\stackrel{0}{1}\end{array}$ & $\begin{array}{l}\overrightarrow{8} \\
\dot{0} \\
\dot{0}\end{array}$ & $\begin{array}{l}\underset{H}{J} \\
\stackrel{0}{0} \\
0\end{array}$ & $\begin{array}{l}\vec{F} \\
0 \\
0 \\
0\end{array}$ & \\
\hline $\begin{array}{l}\underset{\Xi}{\Xi} \\
\frac{\square}{-1}\end{array}$ & 呑 & $\stackrel{8}{\infty}$ & 走 & $\begin{array}{l}10 \\
10 \\
1\end{array}$ & ภิ & $\alpha$ \\
\hline$\underset{2}{\xi \mid l \infty}$ & भ̊ & 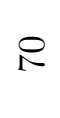 & 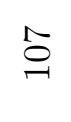 & $\stackrel{\Gamma}{\sim}$ & $\stackrel{\infty}{1}$ & 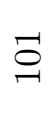 \\
\hline 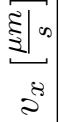 & $\Xi$ & శి & 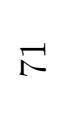 & $\stackrel{\infty}{\stackrel{\infty}{=}}$ & $\stackrel{\mathscr{D}}{\stackrel{10}{-1}}$ & $\ddot{\infty}$ \\
\hline 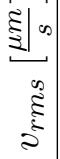 & $\stackrel{12}{7}$ & $\infty$ & $\stackrel{\overbrace{}}{\mathcal{I}}$ & ๙े & 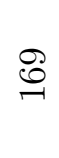 & $\bar{\pi}$ \\
\hline$\frac{\Xi}{\sigma}$ & $\begin{array}{c}\hat{n} \\
\text { on }\end{array}$ & $\stackrel{\rho}{\stackrel{\rho}{\circ}}$ & $\underset{\infty}{\infty}$ & $\begin{array}{l}8 \\
\stackrel{8}{10} \\
\rightarrow+1\end{array}$ & 1 & \\
\hline$\AA^{\Sigma}$ & $\begin{array}{l}\infty \\
19 \\
\infty\end{array}$ & $\begin{array}{l}0 \\
10 \\
\infty\end{array}$ & $\begin{array}{l}\infty \\
\infty \\
\infty \\
\infty\end{array}$ & $\begin{array}{l}0 \\
10 \\
\infty\end{array}$ & $\begin{array}{l}10 \\
10 \\
\infty\end{array}$ & a \\
\hline $\mathscr{S}$ & $\stackrel{\sim}{\stackrel{N}{-}}$ & $\stackrel{\sim}{\stackrel{\Omega}{\sigma}}$ & $\stackrel{\curvearrowright}{\sigma}$ & $\stackrel{\sim}{\stackrel{\sim}{\sigma}}$ & $\stackrel{\infty}{\sigma}$ & 5 \\
\hline 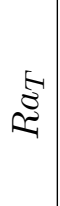 & $\begin{array}{l}\stackrel{8}{0} \\
\dot{1} \\
\dot{8} \\
0 \\
\dot{+} \\
1\end{array}$ & $\begin{array}{l}\stackrel{8}{\circ} \\
\stackrel{-}{0} \\
\dot{\infty} \\
\stackrel{0}{\circ} \\
-1\end{array}$ & $\begin{array}{l}28 \\
\stackrel{1}{1} \\
\dot{0} \\
0 \\
0 \\
0\end{array}$ & $\begin{array}{l}80 \\
\stackrel{1}{1} \\
\dot{8} \\
\dot{1} \\
\dot{1}\end{array}$ & 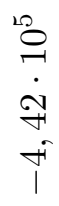 & $\sigma$ \\
\hline$\frac{\bar{E}}{\bar{y}}$ & $\underset{i}{\stackrel{+3}{L}}$ & $\stackrel{+\infty}{\rightarrow-1}$ & $\begin{array}{l}\infty \\
\infty \\
0 \\
\end{array}$ & 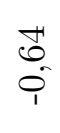 & $\begin{array}{l}\text { is } \\
0 \\
0 \\
1\end{array}$ & is \\
\hline 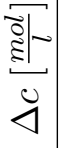 & $\begin{array}{l}+ \\
0 \\
0\end{array}$ & $\begin{array}{l}\overrightarrow{0} \\
0 \\
0\end{array}$ & $\begin{array}{l}\overrightarrow{0} \\
0 \\
0\end{array}$ & $\begin{array}{l}\overrightarrow{0} \\
0 \\
0\end{array}$ & $\begin{array}{l}\sigma_{0}^{+} \\
0\end{array}$ & $\bar{c}$ \\
\hline $\begin{array}{l}\vec{\Xi} \\
\Xi \\
\Xi \\
\Xi \\
z\end{array}$ & $\begin{array}{l}\stackrel{N}{\sim} \\
\stackrel{\infty}{O} \\
\stackrel{0}{\sim} \\
\stackrel{\sim}{N}\end{array}$ & $\begin{array}{l}\underset{1}{1} \\
\stackrel{\sim}{\sim} \\
\stackrel{1}{\infty} \\
\stackrel{0}{0} \\
\stackrel{N}{N}\end{array}$ & 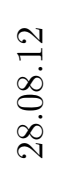 & 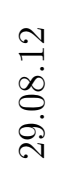 & $\begin{array}{l}\stackrel{N}{7} \\
\stackrel{0}{0} \\
\dot{8}\end{array}$ & 6 \\
\hline
\end{tabular}




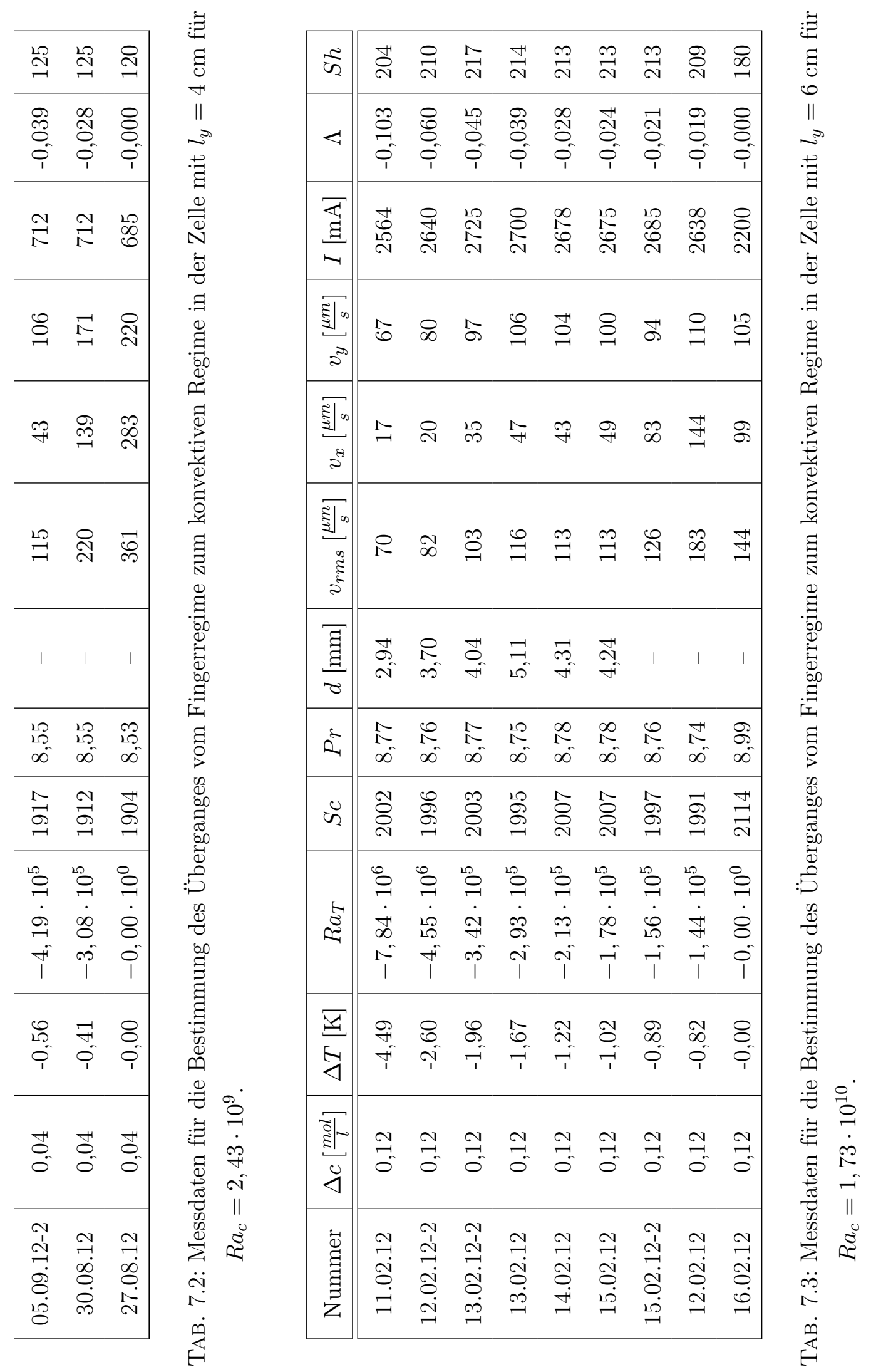




\begin{tabular}{|c|c|c|c|c|c|c|c|}
\hline$\tilde{\omega}$ & $\stackrel{\infty}{\vec{N}}$ & $\stackrel{20}{\sim ึ}$ & $\stackrel{209}{\stackrel{2}{N}}$ & $\stackrel{\mathscr{0}}{\circ}$ & $\begin{array}{l}\stackrel{10}{2} \\
\stackrel{2}{N}\end{array}$ & $\stackrel{\infty}{\sim}$ & $\underset{N}{N}$ \\
\hline$<$ & 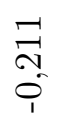 & $\begin{array}{l}0 \\
\infty \\
0 \\
0 \\
1\end{array}$ & $\begin{array}{l}\infty \\
\stackrel{+}{0} \\
0 \\
0\end{array}$ & $\begin{array}{l}\stackrel{N}{O} \\
0 \\
\\
\end{array}$ & $\begin{array}{l}\hat{N} \\
\hat{O} \\
0 \\
0\end{array}$ & 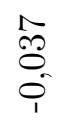 & $\begin{array}{l}8 \\
8 \\
0 \\
0\end{array}$ \\
\hline$\underset{-1}{\stackrel{\Xi}{\Xi}}$ & 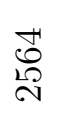 & 유 & $\stackrel{\stackrel{L}{N}}{\stackrel{N}{N}}$ & 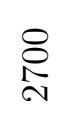 & 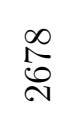 & 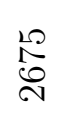 & $\begin{array}{l}10 \\
0 \\
0 \\
\stackrel{0}{N}\end{array}$ \\
\hline$\underbrace{2}_{3}$ & $\stackrel{\leftrightarrow}{\rightarrow}$ & $\ddot{0}$ & $\stackrel{20}{m}$ & 앙 & $\begin{array}{l}\text { N } \\
\infty \\
\infty\end{array}$ & $\stackrel{\Re}{\stackrel{9}{+}}$ & $\frac{10}{6}$ \\
\hline$\underbrace{(5)}_{2^{*}}$ & ని & $\sqrt{20}$ & 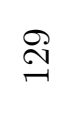 & $\stackrel{\varrho}{N}$ & 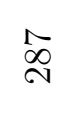 & $\stackrel{N}{\mathcal{H}}$ & $\underset{i 0}{\stackrel{N}{2}}$ \\
\hline 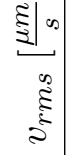 & $\stackrel{\infty}{\stackrel{\infty}{\rightarrow}}$ & 드 & $\stackrel{\infty}{\infty}$ & $\stackrel{\infty}{\stackrel{\infty}{\mathscr{f}}}$ & $\stackrel{N}{\stackrel{N}{F}}$ & $\frac{\infty}{0}$ & $\stackrel{\sim}{\not}$ \\
\hline$\frac{\bar{\Xi}}{\sigma}$ & 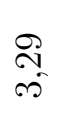 & \begin{tabular}{l}
$\stackrel{\curvearrowright}{\sim}$ \\
\multirow{\leftarrow}{*}{}
\end{tabular} & $\begin{array}{l}\infty \\
10 \\
10\end{array}$ & 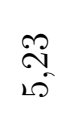 & $\begin{array}{l}\text { N } \\
\text { in }\end{array}$ & I & 1 \\
\hline 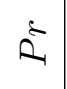 & $\begin{array}{l}\overrightarrow{0} \\
\infty\end{array}$ & $\begin{array}{l}\mathscr{b} \\
\infty\end{array}$ & $\begin{array}{l}\overline{6} \\
\infty\end{array}$ & $\underset{\infty}{8}$ & $\begin{array}{l}0.9 \\
0 \\
0\end{array}$ & $\begin{array}{l}\text { రె } \\
\infty\end{array}$ & $\begin{array}{l}\mathscr{6} \\
\infty\end{array}$ \\
\hline$\ddot{\sim}$ & $\stackrel{30}{\stackrel{2}{2}}$ & 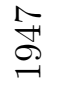 & $\stackrel{\circ}{\stackrel{\circ}{g}}$ & $\begin{array}{l}\mathscr{L} \\
\stackrel{2}{-}\end{array}$ & $\stackrel{\circ}{\stackrel{\circ}{\rightarrow}}$ & $\underset{\rightrightarrows}{\vec{\sigma}}$ & $\stackrel{0}{\stackrel{H}{\sigma}}$ \\
\hline $\overrightarrow{\mathcal{S}}$ & $\begin{array}{l}\stackrel{1}{\circ} \\
\stackrel{1}{*} \\
\dot{N} \\
\text { ô } \\
i\end{array}$ & 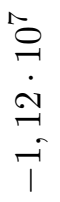 & 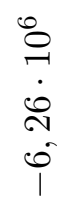 & 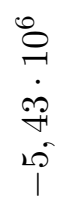 & $\begin{array}{l}8 \\
\stackrel{0}{+} \\
\dot{D} \\
\infty \\
\dot{0} \\
\dot{1}\end{array}$ & 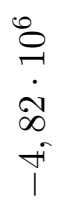 & $\begin{array}{l}8 \\
0 \\
\dot{8} \\
\dot{8} \\
0 \\
1\end{array}$ \\
\hline $\begin{array}{c}\bar{E} \\
\bar{y}\end{array}$ & $\begin{array}{l}8 \\
\stackrel{8}{+} \\
+i\end{array}$ & $\stackrel{\infty}{\infty}$ & $\underset{1}{20}$ & $\begin{array}{l}\vec{\sigma} \\
\dot{0}\end{array}$ & $\begin{array}{l}\vec{\infty} \\
0 \\
0\end{array}$ & $\begin{array}{l}\vec{\infty} \\
0 \\
0 \\
\end{array}$ & $\begin{array}{l}8 \\
0 \\
0\end{array}$ \\
\hline $\begin{array}{l}\stackrel{\vec{g} \mid}{u} \\
\dot{y}\end{array}$ & $\begin{array}{l}0 \\
8 \\
0\end{array}$ & $\begin{array}{l}8 \\
8 \\
0\end{array}$ & $\underset{0}{0}$ & $\begin{array}{l}0 \\
0 \\
0\end{array}$ & 8 & $\begin{array}{l}0 \\
8 \\
0\end{array}$ & $\begin{array}{l}0 \\
0 \\
0\end{array}$ \\
\hline $\begin{array}{l}\vec{\Xi} \\
\stackrel{\Xi}{\Xi} \\
\Xi \\
z\end{array}$ & $\begin{array}{l}\stackrel{\sim}{\mathcal{I}} \\
\stackrel{0}{0} \\
\stackrel{1}{\circ} \\
\stackrel{0}{0}\end{array}$ & $\begin{array}{l}\stackrel{\sim}{\Im} \\
\stackrel{-}{-} \\
\stackrel{0}{0}\end{array}$ & 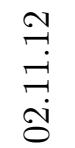 & $\stackrel{\overbrace{}}{\Im}$ & $\begin{array}{l}\stackrel{\sim}{ت} \\
ت \\
ت \\
\ddot{g}\end{array}$ & 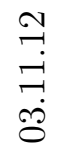 & 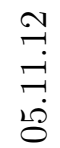 \\
\hline
\end{tabular}

\begin{tabular}{|c|c|c|c|c|c|c|c|c|}
\hline દ్రి & $\tilde{\omega}$ & $\underset{m}{m}$ & 年 & $\stackrel{m}{m}$ & $\stackrel{\infty}{\stackrel{\infty}{\sharp}}$ & 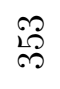 & $\begin{array}{l}10 \\
10 \\
60\end{array}$ & 芯 \\
\hline S & $<$ & $\begin{array}{l}0 \\
\stackrel{2}{二} \\
\stackrel{0}{0}\end{array}$ & 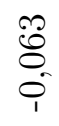 & $\begin{array}{l}\infty \\
2 \\
0 \\
0 \\
0 \\
1\end{array}$ & $\begin{array}{l}\stackrel{g}{+} \\
0 \\
0 \\
\end{array}$ & $\begin{array}{l}\infty \\
\stackrel{0}{0} \\
\stackrel{0}{0}\end{array}$ & \begin{tabular}{l}
\multirow{H}{0}{} \\
0 \\
0 \\
1
\end{tabular} & $\begin{array}{l}\vec{F} \\
0 \\
0 \\
0\end{array}$ \\
\hline & $\frac{\bar{\Xi}}{-}$ & $\stackrel{8}{\stackrel{\leftrightarrow}{े}}$ & $\begin{array}{l}\infty \\
\stackrel{\infty}{*}\end{array}$ & $\begin{array}{l}\stackrel{10}{N} \\
\stackrel{N}{N}\end{array}$ & 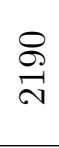 & $\stackrel{20}{\stackrel{2}{N}}$ & 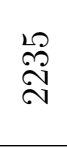 & 今ึ \\
\hline & 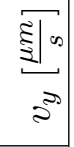 & $R$ & 8 & $\sigma$ & $\stackrel{\Re}{0}$ & $\vec{\sim}$ & $\stackrel{\infty}{=}$ & $\stackrel{\vec{\sigma}}{\sigma}$ \\
\hline & 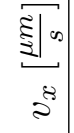 & $\stackrel{0}{-1}$ & $\vec{\sim}$ & নิ & $\stackrel{2}{2}$ & ํำ & 会 & 量 \\
\hline 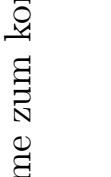 & 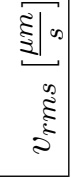 & $\vec{\infty}$ & $\bar{\sigma}$ & $\stackrel{12}{\Omega}$ & $\stackrel{8}{\stackrel{1}{2}}$ & 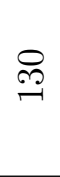 & 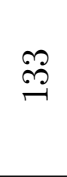 & $\underset{1}{\stackrel{0}{0}}$ \\
\hline$\overline{0}$ & $\begin{array}{c}\bar{\Xi} \\
\frac{\Xi}{\sigma} \\
\end{array}$ & $\begin{array}{l}R \\
\text { in }\end{array}$ & 今. & $\begin{array}{l}\hat{\infty} \\
\infty \\
+\end{array}$ & के & $\stackrel{\overrightarrow{0}}{+}$ & $\stackrel{\infty}{20}$ & $\underset{10}{8}$ \\
\hline & 2 & $\frac{R}{\infty}$ & $\begin{array}{l}\infty \\
1 \\
\infty\end{array}$ & $\begin{array}{l}0 \\
\infty \\
\infty\end{array}$ & $\begin{array}{l}N \\
\infty\end{array}$ & $\begin{array}{l}0 \\
\infty\end{array}$ & $\begin{array}{l}\frac{10}{10} \\
\infty\end{array}$ & $\begin{array}{l}0 \\
\infty \\
\infty\end{array}$ \\
\hline$=$ & $\tilde{\omega}$ & $\stackrel{\sim}{\stackrel{\sim}{*}}$ & $\stackrel{\infty}{\stackrel{ి}{ి}}$ & $\stackrel{\infty}{\mathscr{O}}$ & ङ્م & $\stackrel{ }{\circ}$ & $\stackrel{\mathscr{L}}{\mathscr{g}}$ & 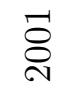 \\
\hline$\frac{g_{0}^{2}}{0}$ & $\stackrel{5}{2}$ & 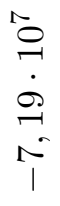 & 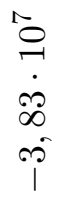 & 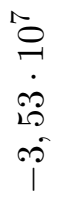 & 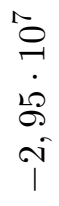 & 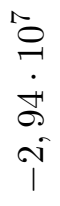 & $\begin{array}{l}\hat{0} \\
\stackrel{1}{0} \\
\dot{2} \\
0 \\
1 \\
1\end{array}$ & 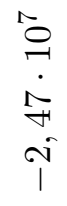 \\
\hline & $\frac{\Sigma}{\Xi}$ & $\stackrel{\sim}{2}$ & $\begin{array}{l}\stackrel{\infty}{\sim} \\
\stackrel{N}{\sim}\end{array}$ & $\begin{array}{l}\text { iै } \\
\text { iิ } \\
\text { Tे }\end{array}$ & $\underset{\text { i }}{ت}$ & \begin{tabular}{l}
0 \\
\multirow{1}{*}{} \\
ô
\end{tabular} & $\underset{1}{\infty}$ & $\stackrel{5}{r}$ \\
\hline 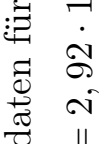 & $\begin{array}{l}\stackrel{\vec{g} \mid-1}{u} \\
\dot{y}\end{array}$ & $\stackrel{\text { If }}{\stackrel{0}{0}}$ & $\stackrel{\sim}{\tilde{O}}$ & Эै & $\stackrel{\tilde{I}}{\tilde{0}}$ & $\stackrel{?}{\tilde{0}}$ & $\stackrel{\sim}{\stackrel{2}{0}}$ & $\stackrel{\sim}{3}$ \\
\hline 華 & 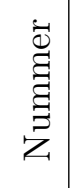 & $\begin{array}{l}\stackrel{v}{\exists} \\
\stackrel{-}{\circ} \\
\stackrel{0}{\sim} \\
\stackrel{\sim}{N}\end{array}$ & 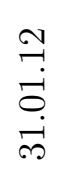 & 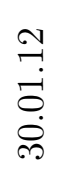 & 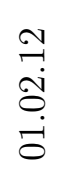 & 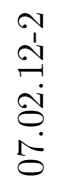 & 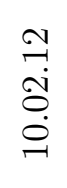 & 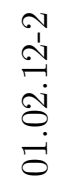 \\
\hline
\end{tabular}




\begin{tabular}{|c|c|c|c|c|c|}
\hline 点 & $\begin{array}{l}\text { is } \\
\text { is }\end{array}$ & $\begin{array}{l}0 \\
\stackrel{0}{\infty} \\
\end{array}$ & 㓓 & $\stackrel{20}{\infty}$ & $\overrightarrow{\widetilde{\sim}}$ \\
\hline $\begin{array}{l}\stackrel{0}{0} \\
0 \\
0 \\
\end{array}$ & $\begin{array}{l}\hat{n} \\
\tilde{0} \\
0 \\
0 \\
1\end{array}$ & $\begin{array}{l}\overrightarrow{0} \\
\stackrel{0}{0} \\
0\end{array}$ & 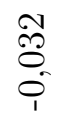 & $\begin{array}{l}\stackrel{N}{0} \\
0 \\
0 \\
1\end{array}$ & $\begin{array}{l}8 \\
8 \\
0 \\
0 \\
1\end{array}$ \\
\hline 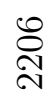 & $\stackrel{\stackrel{2}{N}}{\underset{N}{N}}$ & $\underset{\text { 유 }}{\stackrel{\text { N }}{1}}$ & $\underset{⿱ 乛}{\stackrel{1}{N}}$ & $\begin{array}{l}\stackrel{10}{0} \\
\stackrel{1}{N}\end{array}$ & 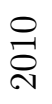 \\
\hline$\stackrel{\stackrel{L}{N}}{\sim}$ & $\vec{I}$ & $\stackrel{\vec{m}}{\rightarrow}$ & $\underset{\exists}{\exists}$ & $\underset{ }{\stackrel{ }{4}}$ & $\stackrel{20}{=}$ \\
\hline$\stackrel{12}{7}$ & $\mathscr{o}$ & 8 & $\overrightarrow{0}$ & $\stackrel{\infty}{\stackrel{\infty}{\sim}}$ & $\stackrel{\overbrace{}}{\sim}$ \\
\hline$\vec{\infty}$ & $\stackrel{\sim}{\sim}$ & $\stackrel{0}{\stackrel{1}{1}}$ & $\stackrel{\text { m }}{\rightarrow}$ & $\stackrel{\mathscr{S}}{\rightarrow}$ & $\stackrel{0}{N}$ \\
\hline$\underset{10}{\infty}$ & $\begin{array}{l}10 \\
10 \\
10\end{array}$ & $\begin{array}{l}0 \\
20 \\
10\end{array}$ & 1 & 1 & 1 \\
\hline $\begin{array}{l}0 \\
1 \\
\infty\end{array}$ & $\begin{array}{l}0 \\
\infty\end{array}$ & $\begin{array}{l}\kappa \\
\infty\end{array}$ & $\begin{array}{l}0 \\
\infty\end{array}$ & $\begin{array}{l}\infty \\
\infty \\
\infty\end{array}$ & 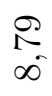 \\
\hline$\stackrel{\mathscr{g}}{\mathscr{g}}$ & 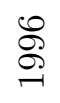 & ஜ̊̊ి & $\stackrel{\mathscr{S}}{\stackrel{\leftrightarrow}{二}}$ & 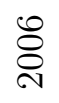 & $\stackrel{m}{\stackrel{\infty}{\circ}}$ \\
\hline ro & $\stackrel{1}{\circ}$ & so & $\stackrel{1}{\circ}$ & 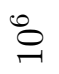 & $\stackrel{8}{\circ}$ \\
\hline F & $\stackrel{\vec{N}}{ }$ & $\mathscr{2}$ & $\dot{8}$ & 穴 & 8 \\
\hline î & $\stackrel{\sim}{i}$ & $\stackrel{\mathfrak{i}}{i}$ & i & $\varphi_{1}^{0}$ & $0_{1}^{\circ}$ \\
\hline$\stackrel{N}{i}$ & 8 & 감 & 유 & 요 & 8 \\
\hline-5 & $\hat{i}$ & $\hat{i}$ & $i$ & i & i \\
\hline$\stackrel{\sim}{\stackrel{\sim}{0}}$ & $\stackrel{\sim}{\exists}$ & $\stackrel{\stackrel{N}{二}}{\breve{0}}$ & $\stackrel{\sim}{\leftrightarrows}$ & $\stackrel{\sim}{\stackrel{2}{0}}$ & $\stackrel{1}{7}$ \\
\hline$\stackrel{\mathcal{F}}{\mathcal{F}}$ & $\stackrel{\sim}{\sim}$ & $\stackrel{\sim}{\sim}$ & $\stackrel{\sim}{\sim}$ & $\stackrel{\sim}{\sim}$ & $\stackrel{\mathcal{N}}{\sim}$ \\
\hline$\varepsilon$ & $\stackrel{d}{0}$ & $\stackrel{1}{0}$ & $\stackrel{d}{0}$ & ฮ่ & $\stackrel{\delta}{0}$ \\
\hline$\hat{c}$ & $\dot{8}$ & $\dot{0}$ & $\dot{0}$ & $\dot{\partial}$ & $\dot{8}$ \\
\hline
\end{tabular}

年

\begin{tabular}{|c|c|c|c|c|c|c|c|c|c|c|}
\hline$\tilde{\omega}$ & $\stackrel{\sim}{-1}$ & 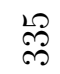 & 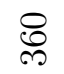 & 足 & 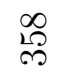 & ڤి & $\begin{array}{l}10 \\
10 \\
10\end{array}$ & 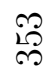 & $\stackrel{g}{\mathrm{H}}$ & 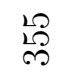 \\
\hline$<$ & $\begin{array}{l}\infty \\
0 \\
0 \\
0 \\
0\end{array}$ & 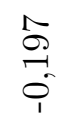 & $\begin{array}{l}0 \\
\stackrel{0}{2} \\
\stackrel{0}{0}\end{array}$ & $\begin{array}{l}\infty \\
\stackrel{0}{0} \\
\stackrel{0}{0}\end{array}$ & 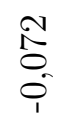 & $\begin{array}{l}8 \\
8 \\
0 \\
0\end{array}$ & 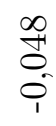 & \begin{tabular}{l}
$\infty$ \\
\multirow{0}{*}{} \\
0 \\
1
\end{tabular} & $\begin{array}{l}\stackrel{20}{0} \\
0 \\
0 \\
1\end{array}$ & $\begin{array}{l}\text { JH } \\
\text { O } \\
0 \\
\end{array}$ \\
\hline$\frac{\vec{\Xi}}{-}$ & 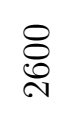 & 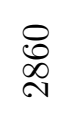 & \&. & 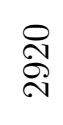 & 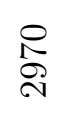 & ণิ) & $\begin{array}{l}\stackrel{2}{2} \\
\stackrel{2}{2}\end{array}$ & 유 & $\begin{array}{l}\stackrel{8}{\circ} \\
\stackrel{2}{2}\end{array}$ & 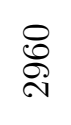 \\
\hline
\end{tabular}

:

ஸ

हा

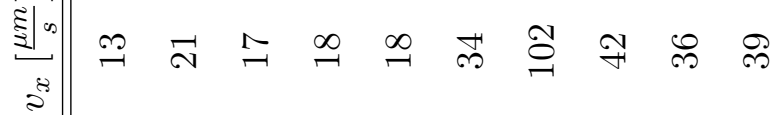

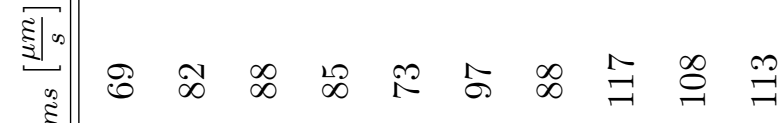

घ

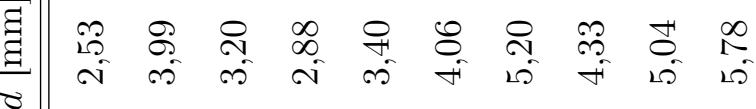

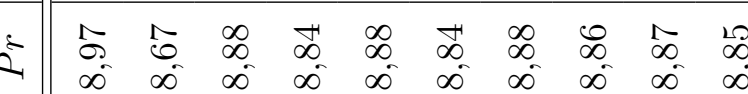

น

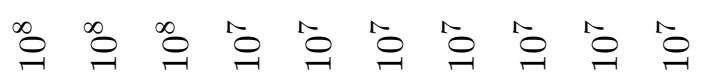

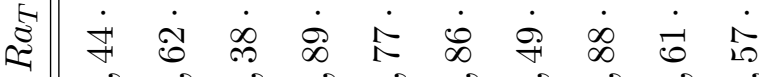

๙

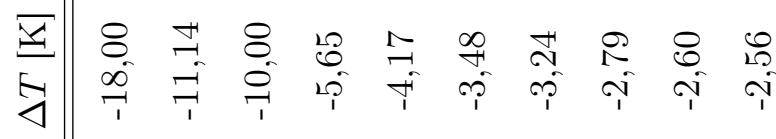

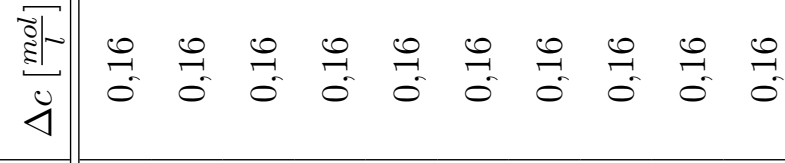

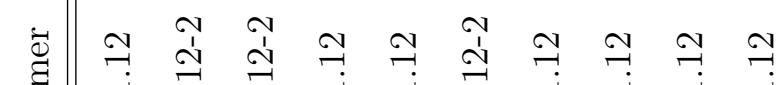

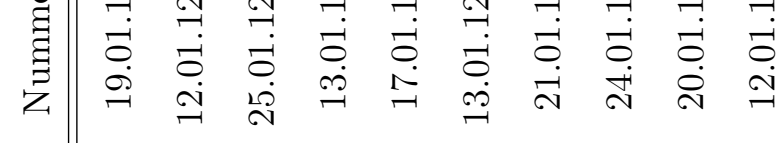




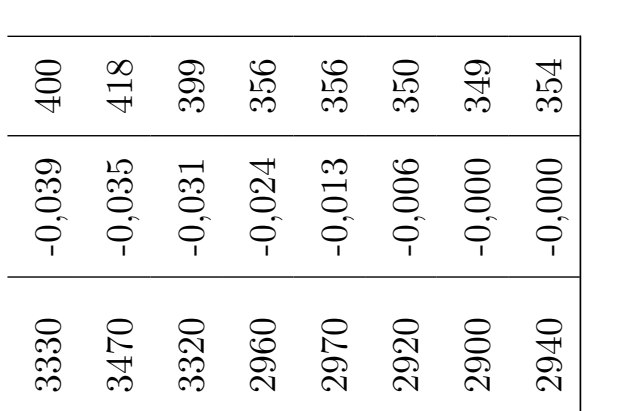

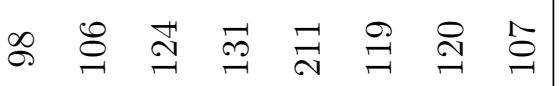

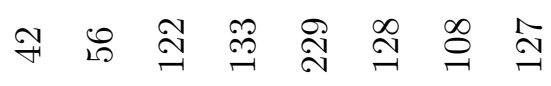

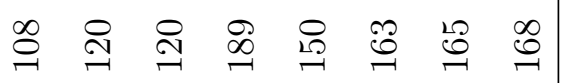

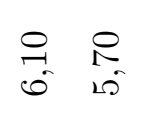

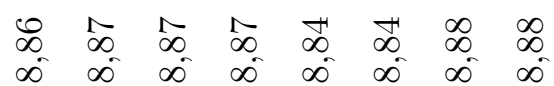

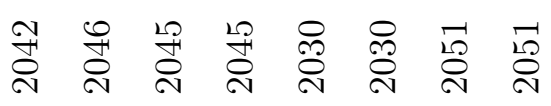

包总总总总邑五导

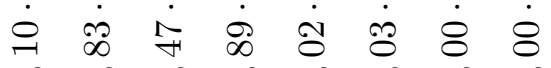

कि i

๙

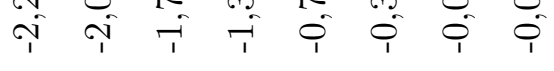

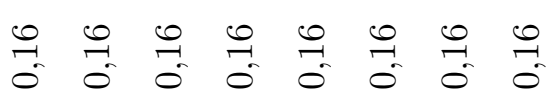

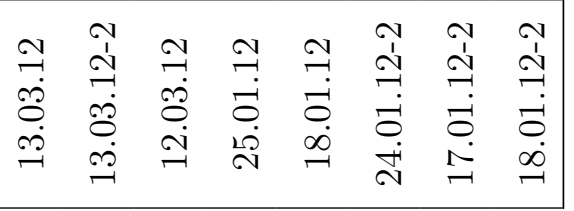

进

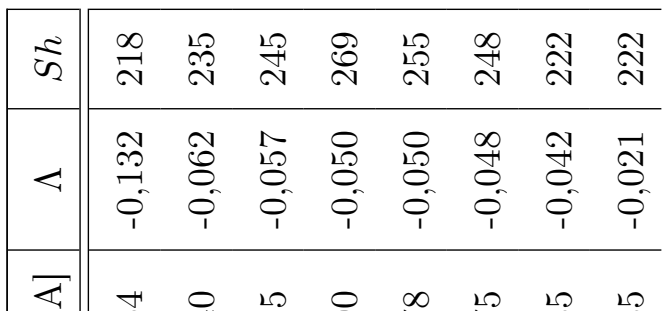

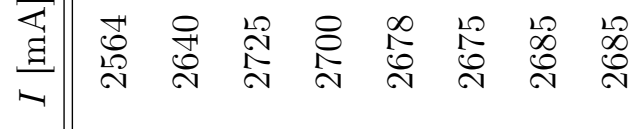

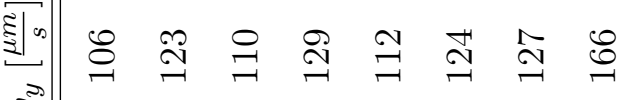

䜿

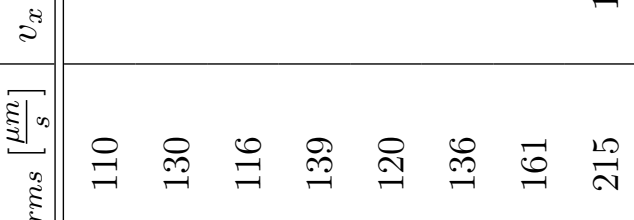

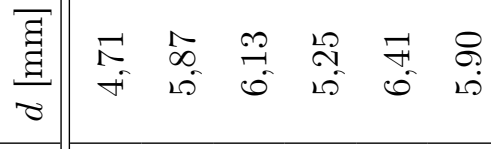

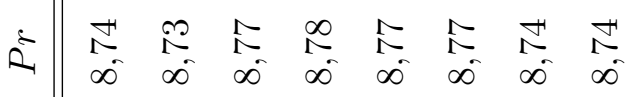

น

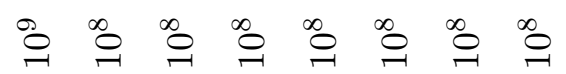

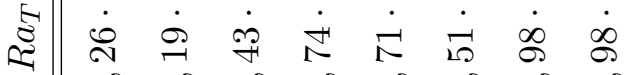

i

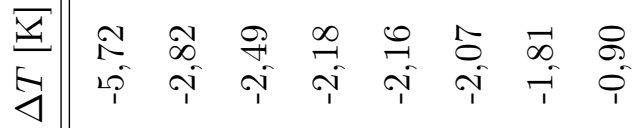

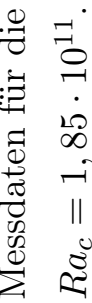

$\stackrel{\ddot{\leftrightarrow}}{\sim}$

䍃 
7 Apendix

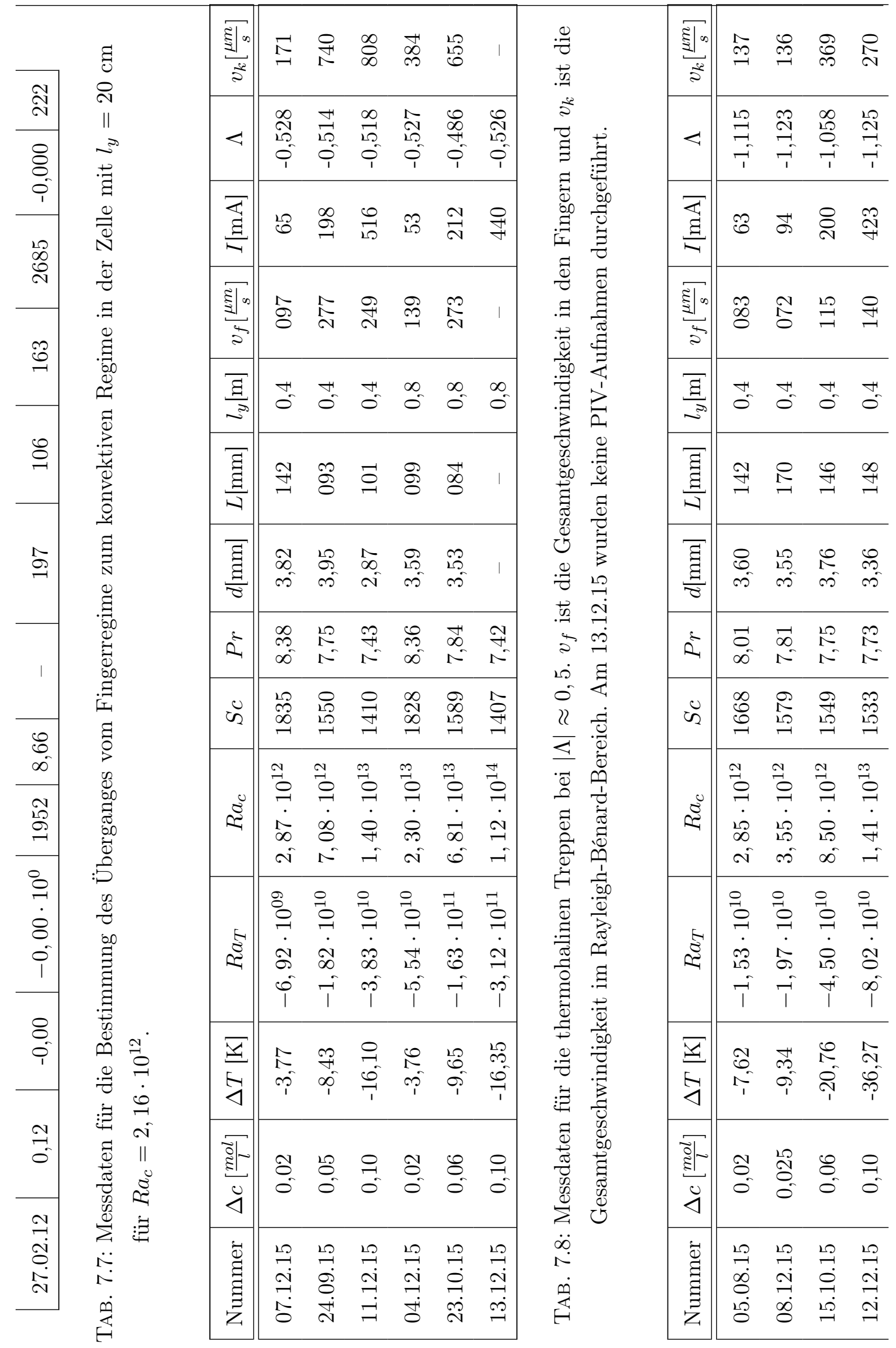




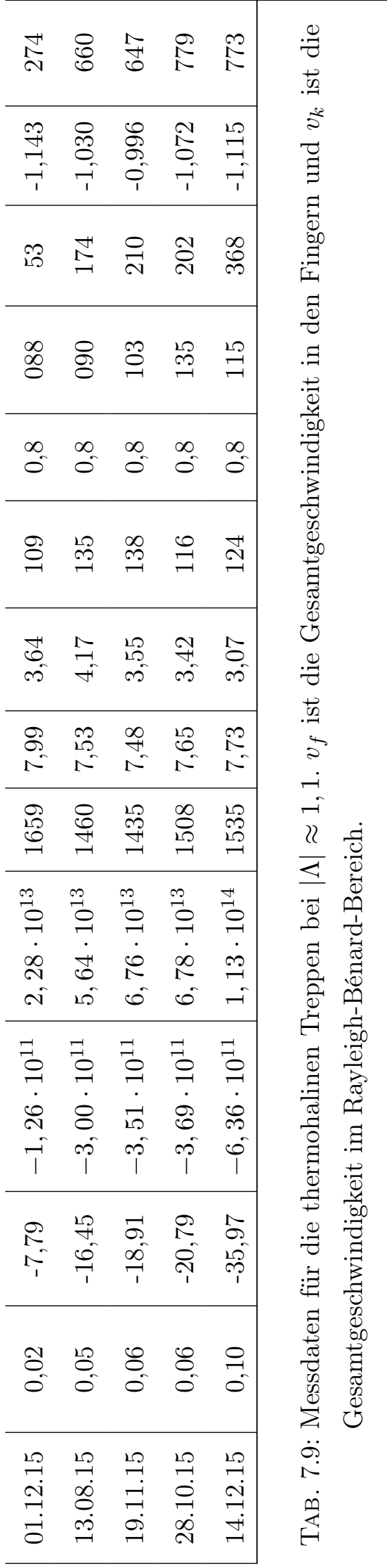




\subsection{Gleichungen zur Bestimmung der Stoffeigenschaften}

Die Stoffeigenschaften werden nicht wie in der Boussinesqnäherung als konstant angenommen, um die aus dieser Näherung entstehenden Fehler zu vermeiden. Daher wird hier beschrieben, wie alle Materialeigenschaften berechnet werden. Die Formeln stammen aus [Chiang and Goldstein, 1992] und die Referenzwerte aus [CRC, 2000]. $c_{1}$ ist die Konzentration der Kupferionen und $c_{2}=1 \frac{\mathrm{mol}}{l}$ ist die Konzentration des Leitsalzes. $\Theta=\left(T_{\text {oben }}-T_{\text {unten }}\right) / 2-25^{\circ} \mathrm{C}$.

- Dichte:

$$
\begin{array}{r}
\varrho\left[\frac{\mathrm{kg}}{\mathrm{m}^{3}}\right]=999,448+148,07 c_{1}+60,816 c_{2}-0,4246 \Theta+1,51 c_{1}^{2}-0,7043 c_{2}^{2} \\
-4,56 c_{1} c_{2}-4,47 \cdot 10^{-3} \Theta^{2}-6 \cdot 10^{-6} c_{1} \Theta+1,81 \cdot 10^{-2} c_{2} \Theta
\end{array}
$$

- Viskosität

$$
\begin{aligned}
\mu\left[\frac{\mathrm{m}^{2}}{\mathrm{~s}}\right]=(899,54 & +453,7 c_{1}+140,63 c_{2}-19,235 \Theta+232,4 c_{1}^{2}+28,94 c_{2}^{2} \\
& \left.+94,96 c_{1} c_{2}+0,321 \Theta^{2}-15,04 c_{1} \Theta-4,953 c_{2} \Theta\right) 10^{-6}
\end{aligned}
$$

- spezifische Wärmekapazität

$$
c_{p}\left[\frac{\mathrm{J}}{\mathrm{kgK}}\right]=4178-174,6 c_{2}
$$

- Wärmeleitfähigkeit

$$
k_{T}\left[\frac{\mathrm{J}}{\mathrm{sKm}}\right]=0,52077+0,0049033 c_{2}
$$

- Diffusionskoeffizienten

$$
\begin{gathered}
\kappa_{c}\left[\frac{\mathrm{m}^{2}}{\mathrm{~s}}\right]=\frac{2,09}{\mu}(T+273,15) 10^{-15} \\
\kappa_{T}\left[\frac{\mathrm{m}^{2}}{\mathrm{~s}}\right]=\frac{k_{T}}{\varrho c_{p}}
\end{gathered}
$$


- Transportzahl

$$
t_{C u^{2+}}=\left(0,2633-0,1020 c_{2}\right) c_{1}
$$

- Dichtevariationskoeffizienten:

$$
\begin{gathered}
\alpha_{c}\left[\frac{1}{\mathrm{~mol}}\right]=\frac{1}{\varrho}\left(148,07+3.02 c_{1}-4,56-0.06 T\right) \\
\alpha_{T}\left[\frac{1}{\mathrm{~K}}\right]=\frac{1}{\varrho}\left(-0,4246-8,94 \cdot 10^{-7} T-0,06 c_{1}+1,81 \cdot 10^{-2} c_{2}\right)
\end{gathered}
$$

\subsection{Technische Daten}

Im Wesentlichen wurde der Versuchsaufbau von [Hage, 2009] übernommen.

\section{Thermostate}

\begin{tabular}{|c|c|}
\hline Firma & Lauda \\
Typ & Ecoline Kältethermostate Re310 \\
\hline Arbeitstemperaturbereich & $-40^{\circ} \mathrm{C}$ bis $200^{\circ} \mathrm{C}^{\circ}$ \\
Umgebungstemperaturbereich & $5^{\circ} \mathrm{C}$ bis $40^{\circ} \mathrm{C}^{\circ}$ \\
\hline Heizleistung & $2,25 \mathrm{~kW}$ \\
Kühlleistung bei $20^{\circ} \mathrm{C}$ & $0,2 \mathrm{~kW}$ \\
\hline Förderdruck & bis $0,4 \mathrm{bar}$ \\
Förderstrom & bis $17 \frac{1}{\min }$ \\
\hline Einstellgenauigkeit & $0,1^{\circ} \mathrm{C}$ \\
Temperaturkonstanz & $0,2^{\circ} \mathrm{C}$ \\
\hline
\end{tabular}

TAB. 7.10: Daten der beiden verwendeten Thermostate. 


\section{Temperaturmessung}

\section{Thermistoren}

Die Temperatur der Platten wurde mit NTC-Widerständen gemessen. Die Widerstände wurden mittels eines Multimeters ausgelesen und mit Hilfe der Eichung (siehe Abb. 7.10) in Temperaturen umgerechnet. Daher ist es nicht von Belang, dass der genaue Typ der Thermistoren nicht bekannt ist. Beim Multimeter wurden hauptsächlich Widerstände zwischen $1 \mathrm{k} \Omega$ und $10 \mathrm{k} \Omega$ gemessen. Es wurde mittels eines selbstgeschriebenen Programms über eine Schnittstelle angesteuert. Die Daten des Multimeters sind in Tabelle 7.11 aufgelistet.

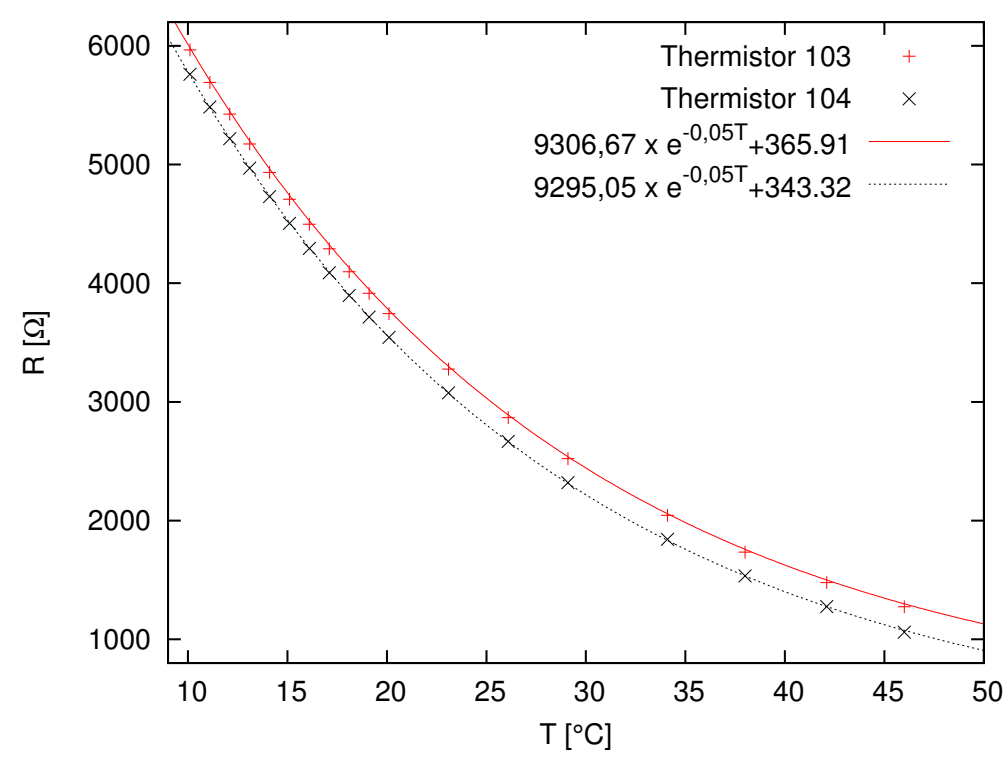

ABB. 7.10: Kalibrierungskurven der Thermistoren. Die Daten für Thermistor 104 sind zur besseren Übersicht um $-200 \Omega$ verschoben wurden. 


\begin{tabular}{|c|c|}
\hline $\begin{array}{c}\text { Firma } \\
\text { Typ }\end{array}$ & Keithley \\
Widerstandsbereiche & Multimeter Modell 2700 \\
& bis $100 \Omega$ \\
bis $1 \mathrm{k} \Omega$ \\
bis $10 \mathrm{k} \Omega$ \\
bis $100 \mathrm{k} \Omega$ \\
bis $1 \mathrm{M} \Omega$ \\
bis $10 \mathrm{M} \Omega$ \\
bis $100 \mathrm{M} \Omega$ \\
\hline Auflösung & $1 \mathrm{ppm}$ vom Messbereich \\
\hline Fehler & $106 \mathrm{ppm}$ für den Messbereich bis $10 \mathrm{k} \Omega$ \\
\hline
\end{tabular}

TAB. 7.11: Daten des verwendeten Multimeters

\section{Thermokristalle}

Die Thermokristalle stammen aus der SLN40 Serie der Firma Hallcrest und sind in einer Schutzkapsel eingebettet. Deren Größe beträgt 50-100 $\mu \mathrm{m}$ im Durchmesser. Die Daten der Thermokristalle sind der Tabelle 7.12 zu entnehmen.

\begin{tabular}{|c|c|c|}
\hline Name & "rot-Start" & "blau Start" \\
\hline $\mathrm{R} 16 \mathrm{CW} 14$ & $16^{\circ} \mathrm{C}$ & $30{ }^{\circ} \mathrm{C}$ \\
$\mathrm{R} 22 \mathrm{CW} 2$ & $22^{\circ} \mathrm{C}$ & $24^{\circ} \mathrm{C}$ \\
\hline
\end{tabular}

TAB. 7.12: Theoretische Daten der verwendeten Thermokristalle. Die Thermokristalle wurden vor jedem Einsatz geeicht, da vor allem der "blau-Start" mit der Zeit driftet. 


\section{PIV-Komponenten}

\section{Laser}

Die Daten des Lasersystems sind der Tabelle 7.13 zu entnehmen. Mittels Linsen, die an das Lasergehäuse angeschraubt werden können, kann der Strahl aufgeweitet werden, um eine Ebene oder die gesamte Zelle zu beleuchten. Dabei lässt sich die Ebene stufenlos um $360^{\circ}$ drehen. Der Laser kann extern getriggert werden (siehe Abschnitt Synchronisation).

\begin{tabular}{|c|c|}
\hline Firma & New Wave Research, Inc \\
Typ & Solo III-15 \\
& Lasersystem - 2 Laser \\
\hline Wellenlänge & $1064 \mathrm{~nm}$ \\
Verdopplung & via KTP-Kristall auf $532 \mathrm{~nm}$ \\
Energie & $50 \mathrm{~mJ}$ \\
Frequenz & $15 \mathrm{~Hz}$ \\
Strahldurchmesser & $3,5 \mathrm{~mm}$ \\
Pulsweite (FWHM) & $3 \mathrm{~nm}$ bis $5 \mathrm{~nm}$ \\
\hline
\end{tabular}

TAB. 7.13: Daten des verwendeten Lasers

\section{Kamera}

Die Spezifikationen der Kamera sind der Tabelle $7.14 \mathrm{zu}$ entnehmen. An die Kamera können noch Objektive angeschraubt werden. Für die PIV-Aufnahmen wurde das "AF Nikkor 50 mm 1:1,8D“ von Nikon benutzt. Die Kamera wird extern getriggert, um mit dem Laser synchron zu laufen (siehe Abschnitt Synchronisation). Gesteuert wird sie über das mitgelieferte Programm "CamWare". 


\begin{tabular}{|c|c|}
\hline $\begin{array}{c}\text { Firma } \\
\text { Typ }\end{array}$ & $\begin{array}{l}\text { pco.imaging } \\
\text { pixelfly qe }\end{array}$ \\
\hline Pixel & $1392(\mathrm{H}) \times 1024(\mathrm{~V})$ \\
\hline Pixelgröße & $6,45 \mu \mathrm{m} \times 6,45 \mu \mathrm{m}$ \\
\hline CCD-Sensorscanbereich & $9,0 \mathrm{~mm} \times 6,6 \mathrm{~mm}$ \\
\hline maximale Frequenz & $12 \mathrm{fps}$ bis $23 \mathrm{fps}$ \\
\hline Farben & $\mathrm{s} / \mathrm{w}$ \\
\hline Wellenlängenbereich & $290 \ldots 1000 \mathrm{~nm}$ \\
\hline maximale Empfindlichkeit & $62 \%$ bei $500 \mathrm{~nm}$ \\
\hline Software & CamWare \\
\hline
\end{tabular}

TAB. 7.14: Daten der verwendeten Kamera

\section{Synchronisation}

Die Synchronisation sorgt dafür, dass die Kamera und der Laser synchron mit einer vorgegebenen Frequenz laufen. Dabei wurde der von der Firma Intelligent Laser Application GmbH hergestellte "mini-PIV-Synchronizer" verwendet. Die Frequenz, die Helligkeit der Laserpulse und die Auslesefrequenz der Kamera können manuell gesteuert werden.

\section{Software}

Mittels der Software "VidPIV 4.1" von Intelligent Laser Application GmbH können die von der Kamera aufgenommenen Bilder ausgewertet werden. Dabei lassen sich alle Teilschritte modular zusammenstellen. Ein Maßstab, der Zeitabstand zwischen den Bildern und die gewünschten Filter müssen manuell eingestellt werden. Es gibt einen globalen Filter und einen lokalen Filter, sowie die Option zu interpolieren. Am Ende kann man sich das Ergebnis als Vektorkarte oder als Datei mit den Daten ausgeben lassen. Zudem kann man die Vektorkarte noch als Konturplot darstellen lassen. 


\section{Tracerpartikel}

Die Daten der Tracerpartikel sind der Tabelle $7.15 \mathrm{zu}$ entnehmen.

\begin{tabular}{|c|c|}
\hline $\begin{array}{c}\text { Firma } \\
\text { Bezeichnung }\end{array}$ & $\begin{array}{c}\text { Dantec Dynamics } \\
\text { Polyamid Seeding Particles }\end{array}$ \\
\hline Material & Polyamide 12 \\
Form & nicht Sphärisch, rund \\
mittlere Größe & $\oslash=50 \mu \mathrm{m}$ \\
Streuung & $30-70 \mu \mathrm{m}$ \\
Dichte & $1,03 \frac{\mathrm{g}}{\mathrm{cm}^{3}}$ \\
Brechungsindex & 1,5 \\
Schmelzpunkt & $175^{\circ} \mathrm{C}$ \\
\hline
\end{tabular}

TAB. 7.15: Daten der verwendeten Tracerpartikel

\section{Sonstige Geräte}

Mittels der Vakuumpumpe "MD 4" von "vacuubrand" wird das Arbeitsfluid vor den Messungen entgast, um Blasenbildung an den Zellwänden zu vermeiden. Dabei sollte man pro Liter Arbeitsfluid mindestens $2 \mathrm{~h}$ lang entgasen. Das Saugvermögen beträgt $3,3 \frac{\mathrm{m}^{3}}{\mathrm{~h}}$. Die Spannungsquelle ist ein "E3644A" von Agilent Technologies mit einen Arbeitsbereich von 0 bis $8 \mathrm{~A}$ und 0 bis $8 \mathrm{~V}$. Die Aufösung beträgt $10 \mathrm{mV}$.

Mittels der Analysewaage "CP64" der Firma "Sartorius" können die Chemikalien auf $1 / 10 \mathrm{mg}$ genau abgewogen werden.

Es wurden folgende Chemikalien verwendet:

- destilliertes Wasser $\left(\mathrm{H}_{2} \mathrm{O}\right)$ : zum Verdünnen und Reinigen

- Kupfersulfat-Pentahydrat $\left(\mathrm{CuSO}_{4} \cdot 5 \mathrm{H}_{2} \mathrm{O}\right)$ : in kristalliner Form zur Herstellung einer Anfangskupferionenkonzentration $c_{0}$ : CAS-Nr.: [7758-99-8]; UN-Nr. 3077

- Schwefelsäure $\left(\mathrm{H}_{2} \mathrm{SO}_{4}\right): 96 \%$ rein, zur Herstellung des Arbeitsfluides: CAS-Nr. [7664-93-9]; UN-Nr. 1830 


\section{Literaturverzeichnis}

[CRC, 2000] (2000). CRC Handbook of Chemistry and Physics. CRC Press LLC, 81 edition.

[Ahlers et al., 2009] Ahlers, G., Grossmann, S., and Lohse, D. (2009). Heat transfer and large scale dynamics in turbulent Rayleigh-Bénard convection. Rev. Mod. Physics, $81(2)$.

[Baines and Gill, 1969] Baines, P. and Gill, A. (1969). On thermohaline convection with linear gradients. J. Fluid Mech., 37(2).

[C.H.Hamann and W.Vielstich, 1980] C.H.Hamann and W.Vielstich (1980). Elektrochemie II. VCH, Weinheim.

[Chiang and Goldstein, 1992] Chiang, H. and Goldstein, R. (1992). Application of the Electrochemical Mass Transfer Technique to the study of Bouyancy-driven Flows. In Transport Phenomena in Heat and Mass Transfer, volume 1. Elsevier, Amsterdam.

[Cooper and Stommel, 1968] Cooper, J. and Stommel, H. (1968). Regularly spaced steps in the main thermohaline near Bermuda. J.Geophys.Res., 73.

[Dabiri and Gharib, 1991] Dabiri, D. and Gharib, M. (1991). Digital particle image thermometry: The method and implementation. Experiments in Fluids, 11:77-86.

[Gerstung, 2010] Gerstung, P. (2010). Numerische 2D-Simulationen chemisch getriebener Konvektion mit drei Ionen Sorten. Master's thesis, Georg-August-Universität, Göttingen.

[Ghajar and Bang, 1993] Ghajar, A. and Bang, K. (1993). Experimental and analytical studies of different methods for producing stratified flows. Energy, 18(4).

[Grand, 1997] Grand, I. (1997). Particle image velocimetry: a review. Proc IMechE, Part C: J Mech Eng Sci, 211. 
[Green, 1974] Green, J. S. A. (1974). Two-dimensional turbulence near the viscous limit. J. Fluid Mech., 62:273-287.

[Grossmann and Lohse, 2001] Grossmann, S. and Lohse, D. (2001). Thermal convection for large Prandtl number. Phys. Rev. Lett., 86.

[Hage, 2003] Hage, E. (2003). Chemisch getriebene Konvektion. Master's thesis, GeorgAugust-Universität, Göttingen.

[Hage, 2009] Hage, E. (2009). Coherent structures in turbulent Rayleigh-Bénard convection. Dissertation zur Erlangung des Doktorgrades der MathematischNaturwissenschaftlichen Fakultät der Georg-August-Universität zu Göttingen.

[Hage and Tilgner, 2010] Hage, E. and Tilgner, A. (2010). High Rayleigh number convection with double diffusive fingers. Phys. Fluids, 22:076603.

[Holyer, 1981] Holyer, J. Y. (1981). On the collective instability of salt fingers. J. Fluid Mech., 110.

[Holyer, 1984] Holyer, J. Y. (1984). The stability of long, steady, two-dimensional salt fingers. J. Fluid Mech., 147.

[Jevons, 1857] Jevons, W. (1857). On the cirrous form of cloud. London, Edinburgh, Dublin Philos. Mag. J. Sci., 4th Ser., 14.

[Kellner and Tilgner, 2014] Kellner, M. and Tilgner, A. (2014). Transition to finger convection in double-diffusive convection. Phys. Fluids, 26.

[Krishnamurti, 1995] Krishnamurti, R. (1995). Low frequency oscillations in turbulent Rayleigh-Benard convection: laboratory experiments. Fluid dynamics research, 16.

[Krishnamurti, 2003] Krishnamurti, R. (2003). Double-diffusive transport in laboratory thermohaline staircases. J. Fluid Mech., 483.

[Lambert and Demenkow, 1988] Lambert, R. and Demenkow, J. (1988). On the vertical transport due to fingers in double diffusive convection. J. Fluid Mech.

[Levich, 1962] Levich, V. (1962). Physicochemical Hydrodynamics. Prentice Hall, New Jersey.

[Linden, 1973] Linden, P. (1973). On the structure of salt fingers. Deep-Sea Research, 20. 
[Maxworthy, 1972] Maxworthy, T. (1972). Experimental and Theoretical Studies of Horizontal Jets in a Stratified Fluid. Proc. Int. Symp. Stratified Flows.

[McDougall and Taylor, 1984] McDougall, T. and Taylor, J. (1984). Flux measurements across a finger interface at low values of the stability ratio. Journal of Marine Research, $42(1)$.

[Merryfield, 2000] Merryfield, W. (2000). Origin of Thermohaline Staircases. J. Phys. Oceanography, 30.

[Müller, 2013] Müller, T. (2013). Numerische Untersuchung von Fingerstrukturen in doppelt-diffusiver Konvektion. Master's thesis, Georg-August-Universität, Göttingen.

[Oster, 1965] Oster, G. (1965). Density Gradients. Scientific American, 213(2).

[Paparella, 2000] Paparella, F. (2000). Fingering convection: interplay of small and large scales. Annals of the New York Academy of Sciences.

[Radko, 2003] Radko, T. (2003). A mechanism for layer formation in a double-diffusive fluid. J. Fluid Mech., 497.

[Radko, 2013] Radko, T. (2013). Double-Diffusive Convection. Cambridge University Press.

[Radko and Stern, 2000] Radko, T. and Stern, M. (2000). Finite-amplitude salt fingers in a vertically bounded layer. J. Fluid Mech.

[Raffel et al., 1998] Raffel, M., Willert, E., and Kompenhans, J. (1998). Particle image velocimetry: a practical guide. Springer.

[Rayleigh, 1883] Rayleigh, L. (1883). Investigation of the character of the equilibrium of an incompressible heavy fluid of variable density. In Proc. London Math. Soc., volume 14 .

[Ruddick, 1983] Ruddick, B. (1983). A practical indicator of the stability of the water column to double-diffusive activity . Deep-Sea Research, 30.

[Schmitt, 1979a] Schmitt, R. (1979a). Flux measurements on salt fingers at an interface. Journal of Marine Research, 37.

[Schmitt, 1979b] Schmitt, R. (1979b). The growth rate of supercritical salt fingers. DeepSea Research, 26(23). 
[Schmitt, 1994] Schmitt, R. (1994). Double diffusion in oceanography. Ann. Rev. Fluid Mech., 26.

[Schmitt et al., 1987] Schmitt, R., Perkins, H., Boyd, J., and Stalcup, M. (1987). CSALT: an investigation of the thermohaline staircase in the western tropical North Atlantic. Deep-Sea Research, 34.

[Schmitt, 1983] Schmitt, R. W. (1983). The characteristics of salt fingers in a variety of fluid systems, including stellar interiors, liquid metals, oceans, and magmas. Phys. Fluids, 26:2373-2377.

[Schmitt, 2011] Schmitt, R. W. (2011). Thermohaline convection at density ratios below one: A new regime for salt fingers. J. Mar. Res., 69:779-795.

[Sreenivas et al., 2009] Sreenivas, K., Sigh, O., and Srinivasan, J. (2009). On the relationship between finger width, velocity, and fluxes in thermohaline convection. Phys. Fluids, 21(2).

[Stellmach et al., 2011] Stellmach, S., Traxler, A., Garaud, P., Brummell, N., and Radko, T. (2011). Dynamics of fingering convection II: The formation of thermohaline staircases. J. Fluid Mech., 677.

[Stern, 1960] Stern, M. (1960). The "Salt-Fountain" and Thermohaline Convection. Tellus, 12(2).

[Stern, 1969] Stern, M. (1969). Collective Instability of salt fingers. J. Fluid Mech., $35(2)$.

[Stern and Turner, 1969] Stern, M. and Turner, J. (1969). Salt fingers and convecting layers. Deep-Sea Research, 16.

[Stommel et al., 1956] Stommel, H., Arons, A., and Blanchard, D. (1956). An oceanographical curiosity: the perpetual salt fountain. Deep-Sea Research, 3.

[Tait and Howe, 1968] Tait, R. and Howe, M. (1968). Some observations of the thermohaline stratification in the deep ocean. Deep-Sea Research, 15.

[Turner, 1965] Turner, J. (1965). The coupled turbulent transports of salt and and heat across a sharp density interface. Int. J. Heat Mass Transfer, 8(5).

[Turner, 1967] Turner, J. (1967). Salt finger across a density interface. Deep-Sea Research, 14 . 
[Turner, 1974] Turner, J. (1974). Double-diffusive Phenomena. Ann. Rev. Fluid Mech., 6.

[Turner and Stommel, 1964] Turner, J. and Stommel, H. (1964). A new case of cinvection in the presence of combined vertical salinity and temperature gradients. Proc Natl Acad Sci USA, 52(1).

[Xia et al., 2002] Xia, K.-Q., Lam, S., and Zhou, S.-Q. (2002). Heat-Flux Measurements in High-Prandtl-Number Turbulent Rayleigh-Bénard Convection. Phys. Rev. Lett., $88(6)$.

[Yang et al., 2015a] Yang, Y., R.Verzicco, and D.Lohse (2015a). From convection rolls to finger convection on double-diffusive turbulence. Proceedings of the National Academy of Sciences of the USA, 113.

[Yang et al., 2015b] Yang, Y., van der Poel, E., Ostilla-Monico, R., Sun, C., Verzicco, R., Grossmann, S., and D.Lohse (2015b). Salinity transfer in bounded double diffusive convection. J. Fluid Mech., 768.

[You, 2002] You, Y. (2002). A global climatological atlas of the Turner angle: implications for double-diffusion and water mass structure. Deep-Sea Research, 49.

[Zodiatis and Gasparini, 1996] Zodiatis, G. and Gasparini, G. (1996). Thermohaline staircase formation in the Tyrrhenian Sea. Deep-Sea Research, 43.

[Zurigat et al., 1990] Zurigat, Y., Bang, K., and Ghajar, A. (1990). Methods for producing linear density gradients in laboratory tanks. Energy, 15(1).

[Özgökmen et al., 1998] Özgökmen, T., Esenkov, O., and Olson, D. (1998). A numerical study of layer formation due to fingers in double-diffusive convection in a verticallybounded domain. Journal of Marine Research. 



\section{Danksagung}





\section{Lebenslauf}

\section{Persönliche Daten}

Name:

geboren am:

Staatsangehörigkeit: deutsch

Familienstand: verheiratet

\section{Schulbildung}

09/1992 - 07/1996 Grundschule Käthe-Kollwitz in Schönebeck/Elbe

08/1996 - 06/2005 Gymnasium: Dr.Carl-Hermann in Schönebeck/Elbe

Abschluss: Abitur

07/2005 - 09/2006 Grundwehrdienst in Minden

\section{Studium}

10/2006 - 09/2009 Bachelorstudium der Physik an der Georg-August-Universität Göttingen Bachelorarbeit: Korrelation bei thermischer Konvektion Abschluss: Bachelor of Science Physik

10/2009 - 05/2012 Masterstudium der Physik an der Georg-August-Universität Göttingen Masterarbeit: Grenzen des Fingerregimes in doppelt-diffusiv getriebener Strömung

Abschluss: Master of Science Physik

seit 06/2012 Promotion zum Thema "Experimentelle Untersuchungen von Fingerströmung und thermohalinen Treppen für instabile Auftriebsverhältnisse" 